\title{
WestVirginiaUniversity
}

THE RESEARCH REPOSITORY @ WVU

Graduate Theses, Dissertations, and Problem Reports

2009

\section{Cooperative wireless communications: Protocol design and performance analysis}

\author{
Kanchan Vardhe \\ West Virginia University
}

Follow this and additional works at: https://researchrepository.wvu.edu/etd

\section{Recommended Citation}

Vardhe, Kanchan, "Cooperative wireless communications: Protocol design and performance analysis" (2009). Graduate Theses, Dissertations, and Problem Reports. 2931.

https://researchrepository.wvu.edu/etd/2931

This Dissertation is protected by copyright and/or related rights. It has been brought to you by the The Research Repository @ WVU with permission from the rights-holder(s). You are free to use this Dissertation in any way that is permitted by the copyright and related rights legislation that applies to your use. For other uses you must obtain permission from the rights-holder(s) directly, unless additional rights are indicated by a Creative Commons license in the record and/ or on the work itself. This Dissertation has been accepted for inclusion in WVU Graduate Theses, Dissertations, and Problem Reports collection by an authorized administrator of The Research Repository @ WVU.

For more information, please contact researchrepository@mail.wvu.edu. 


\title{
Cooperative Wireless Communications: Protocol Design and Performance Analysis
}

\author{
by \\ Kanchan Vardhe \\ Dissertation submitted to the \\ College of Engineering and Mineral Resources \\ at West Virginia University \\ in partial fulfillment of the requirements \\ for the degree of \\ Doctor of Philosophy \\ in \\ Electrical Engineering \\ Daryl Reynolds, Ph.D., Chair \\ Erdogan Gunel, Ph.D. \\ Natalia Schmid, Ph.D. \\ Matthew C. Valenti, Ph.D. \\ Brian Woerner, Ph.D.
}

Lane Department of Computer Science and Electrical Engineering

Morgantown, West Virginia

2009

Keywords: Cooperative Diversity, CDMA, Decode and Forward, Space Time Codes, Information Outage Probability

Copyright 2009 Kanchan Vardhe 


\author{
Abstract \\ Cooperative Wireless Communications: Protocol Design and Performance Analysis \\ by \\ Kanchan Vardhe \\ Doctor of Philosophy in Electrical Engineering \\ West Virginia University \\ Daryl Reynolds, Ph.D., Chair
}

It is well known that multiple antenna wireless communication systems improve the capacity and reliability of wireless communication systems by leveraging spatial diversity. In many wireless applications where it is impractical to implement multiple antennas at the transceivers, cooperation between wireless users wherein single antenna terminals share their resources has been proposed as a viable option to achieve spatial diversity.

The main goal of this research is to characterize the performance bounds for the multiuser, multirelay cooperative communication systems using information theoretic tools and use it to provide guidance in the design of practical systems. The protocols developed in this work either lead to improved performance or make the system design more practical. To this end, we first consider a two-phase user cooperation scheme where users have been allocated orthogonal channels for transmission. We develop a strategy that jointly performs relay selection and power allocation in a decode-and-forward (DF) multiuser cooperative uplink while minimizing the total uplink power such that each user satisfies its data rate. Results indicate that significant cooperation gains could be obtained using a space-time coded cooperative diversity over the wide range of target rates and total number of users when using the proposed joint relay selection and power minimization algorithm. We also propose a two-phase multiuser DF cooperation protocol that operates in an asynchronous CDMA uplink while relaxing the inter-user orthogonality constraint. The protocol developed here leads to fully distributed cooperation where no inter-user coordination is required and greatly simplifies the medium-access control protocol design. We evaluate the information-outage probability performance of the proposed scheme in an underloaded, fully-loaded and overloaded CDMA uplink. Finally, we propose a DF multistage cooperation protocol wherein the transmission between source and destination takes place in $T \geq 2$ equal duration and orthogonal time phases. In the first time phase, the source broadcasts its message which is received by all potential relays. During subsequent time phases, the relays that have successfully decoded the message using information from all previous transmitting relays, transmit a space-time code for the source's message. The non-decoding relays keep accumulating information and transmit in the later stages when they are able to decode the message. The process repeats for $T$ cooperation time phases. We develop an exact analytical expression for the outage probability of multistage cooperation and use it to analyze near-optimal relay locations and the number of cooperation stages needed for the best performance. 
To my parents Girish Vardhe and Geetanjali Vardhe for their sacrifices, planning of our futures and continuous prayers for our welfare. 


\section{Acknowledgments}

I thank God for giving me the strength and courage that has helped me stand right back on my feet, every time I fell.

I have had the privilege and the honor of interacting with so many fine individuals who have contributed in some way or another in bringing this work to fruition, making things at once easier and harder. I take this opportunity to thank them.

First, I would like to thank my advisor, Dr. Daryl Reynolds for his guidance, patience, timely advice and for providing a flexible research environment. I have learnt the basics of research from him, ways to approach and tackle problems and also improved my writing skills.

I am grateful to Dr. Matthew Valenti for the many fruitful and friendly discussions, his cordial support, expert guidance and valuable feedback on the work. His mentoring throughout my graduate studies is deeply appreciated. I express my sincere thanks to Dr. Brian Woerner for his encouragement and support during the course of my program. I am thankful to Dr. Natalia Schmid for many useful interactions and inspiration. I would like to thank Dr. Erdogan Gunel for being on my committee. I have been fortunate to take courses under all of the committee members and their lectures have been instrumental in shaping my understanding of the subject. Lane Graduate Fellowship is also greatly acknowledged for providing research funding and supporting my studies.

I wish to acknowledge and thank Dr. Mark Jerabek for giving me the first opportunity to work as a teaching assistant. My special thanks to Dr. Ronald Klein for providing me a fascinating, open and enjoyable working environment while I was his teaching assistant and for giving me a chance to offer guest lectures for his classes. I would like to thank Dr. Afzel Noore for the most encouraging discussions and insights on the career.

I want to thank my roommates Manasi and Shilpa and other friends at WVU for making my stay at Morgantown more comfortable, enjoyable and a memorable experience. Many 
thanks to Tarannum for her generosity in providing a place to stay during my frequent visits to Morgantown in the last two semesters while I worked on my thesis from out of town. Thanks to my colleagues, Dr. Rohit Seshadri, Dr. Shi Cheng, Ram and Somak for many helpful discussions and lighter moments we shared.

Finally, but most importantly, my most heartfelt gratitude goes to my parents Girish Vardhe and Geetanjali Vardhe for letting me do what I always wanted to do. Their sacrifices, hardwork, unconditional love, trust, patience, and support through these many years have meant more than I can ever express. Sincere thanks to my brother Shantanu and sisterin-law for their love. Waking up in the mornings to see my brother's e-mail with updates on recent and important events back home in India would almost always kick-start my day in United States. I would also like to thank my extended family including grandparents, uncles, aunts, cousins, and close family-friends for their prayers, all of the care, well wishes, love and encouragement. Thanks to my in-laws for their love. To my husband Satchit, I am thankful for his patience, love and companionship. 


\section{Contents}

Dedication $\quad$ iii

Acknowledgments $\quad$ iv

List of Figures $\quad$ viii

List of Tables $\quad$ xi

Notation $\quad$ xii

1 Introduction $\quad 1$

1.1 Dissertation Motivation and Outline . . . . . . . . . . . . . 3

2 Theoretical Background $\quad 7$

2.1 The Wireless Channel . . . . . . . . . . . . . . . . . . . . . . 7

2.2 Multiuser Communications and CDMA . . . . . . . . . . . . 8

2.2.1 Discrete-time Signal Model for Synchronous CDMA . . . . . . . . . . 10

2.2.2 Discrete-time Signal Model for Asynchronous CDMA . . . . . . . . . 12

2.2 .3 Multiuser Detection . . . . . . . . . . . . . . . . . . . . . 14

2.3 Information Theory for Wireless Fading Channels . . . . . . . . . . . . . 18

2.3.1 Entropy (Self Information) . . . . . . . . . . . . . . 18

2.3.2 Mutual Information . . . . . . . . . . . . . . . . . . . . . 19

2.3.3 Unconstrained Channel Capacity and Outage Probability . . . . . . . 19

2.3.4 Capacity Under Modulation Constraints . . . . . . . . . . . . . 23

2.4 Spatial Diversity . . . . . . . . . . . . . . . . . . . 24

2.4.1 Multi-antenna Systems and Space-Time Communications . . . . . . 25

2.4 .2 Cooperative Communications . . . . . . . . . . . . . 28

3 Joint Power Allocation and Relay Selection for Orthogonal Multiuser Cooperation

3.1 Introduction . . . . . . . . . . . . . . . . . . . . . 34

3.2 System Model . . . . . . . . . . . . . . . . . . . . . . . . 36

3.3 Power Consumption in a Non-cooperative Uplink . . . . . . . . . . . . . . . . . 37

3.4 Power Minimization in a User-Cooperative Uplink . . . . . . . . . . . . . . . 37

3.4.1 Power Minimization under Code Combining . . . . . . . . . . . . . . 40

3.4.2 Power Minimization under Diversity Combining . . . . . . . . . . . 42 
3.5 Simulation Results . . . . . . . . . . . . . . . . . . . . . . 44

3.6 Conclusion . . . . . . . . . . . . . . . . . . . 47

4 Performance of Multiuser Cooperation under Non-orthogonal Channels 48

4.1 System Model . . . . . . . . . . . . . . . . . . . . . . . 51

4.1 .1 Conventional CDMA Uplink . . . . . . . . . . . . . . . 51

4.1 .2 Cooperation in a CDMA Uplink . . . . . . . . . . . . . . . 52

4.2 Performance Under Diversity Combining . . . . . . . . . . . . . . . 58

4.2 .1 Underloaded CDMA Uplink . . . . . . . . . . . . . . . . . 59

4.2 .2 Fully-loaded CDMA Upink . . . . . . . . . . . . . . . . . . . 61

4.2 .3 Overloaded CDMA Uplink . . . . . . . . . . . . . . . . 62

4.3 Performance Under Code Combining . . . . . . . . . . . . . . . . . . . 64

4.4 Performance Under Modulation Constraints . . . . . . . . . . . . . . . . 65

4.5 Numerical Results . . . . . . . . . . . . . . . . . . . . . . . . 67

4.6 Practical System Design . . . . . . . . . . . . . . . . . . . . . . . . . . . . . . . . . . . . . . .

4.6.1 DF User Cooperation . . . . . . . . . . . . . . . . . . . . 73

4.6 .2 Adaptive Receiver Design . . . . . . . . . . . . . . . . . . 74

4.6.3 Simulation Results . . . . . . . . . . . . . . . . . . . . 75

4.7 Conclusions . . . . . . . . . . . . . . . . . . . 76

5 The Performance of Multistage Cooperation $\quad 78$

5.1 System Model . . . . . . . . . . . . . . . . . . . . 81

5.2 Mutual information and Outage Probability . . . . . . . . . . . . . 83

5.3 Numerical Results . . . . . . . . . . . . . . . . . . . . 86

5.4 Conclusions . . . . . . . . . . . . . . . . . . . . . 92

6 Summary, Conclusions and Future Work $\quad 94$

6.1 Summary and Conclusions . . . . . . . . . . . . . . . . . . . . . 94

6.2 Ideas for Future Work . . . . . . . . . . . . . . . . . . . . 97

$\begin{array}{lr}\text { References } & 99\end{array}$

A Convex Optimization and Lagrange Multipliers . . . . . . . . . . . . . . . 104

A.1 Convex Sets . . . . . . . . . . . . . . . . . . . . 104

A.2 Convex Functions . . . . . . . . . . . . . . . . . . 104

A.3 Constrained Optimization . . . . . . . . . . . . . . . 105

A.4 Lagrange Multiplier . . . . . . . . . . . . . . . . . . 105

$\begin{array}{lr}\text { Contributions } & 107\end{array}$ 


\section{List of Figures}

2.1 Bit error rate performance of a synchronous CDMA in a Rayleigh flat fading channels for chip-matched filter detector, decorrelating multiuser detector (MUD) and MMSE MUD. Total number of users is 14, processing gain is 15 and random spreading codes are used. . . . . . . . . . . . .

2.2 Capacity of QPSK modulation and the unconstrained Shannon capacity in AWGN channel . . . . . . . . . . . . . . . . . . . . . 23

2.3 Ergodic capacity of MIMO system with different antenna configurations . . . 26

2.4 Channel allocation for the non-cooperative system. . . . . . . . . . . . 30

2.5 Channel allocation for incremental-based cooperative diversity. . . . . . . . . 30

2.6 Channel allocation for space-time coded cooperative diversity. . . . . . . . . 31

3.1 Average power consumption in a uplink as a function of total number of users. The target rate is $R=1 \mathrm{bit} / \mathrm{sec} / \mathrm{Hz}$. We see that incremental redundancy based cooperation improves performance up to about 5 users, while space-time cooperation uniformly outperforms no cooperation or incremental redundancy based cooperation. . . . . . . . . . . . . . . . .

3.2 Average power consumption in a uplink as a function of target rate for no cooperation (nc), incremental redundancy-based cooperative diversity under code combining (cc), and space-time coded cooperative diversity under diversity combining $(\mathrm{dc})$. The figure indicates that for fewer number of users and target rates of interests, the average total power consumption under both incremental redundancy-based and space-time coded cooperative diversity is significantly less than no cooperation. . . . . . . . . . . . . . .

4.1 Space-time coded medium-access control for a) Laneman's protocol, and b) the proposed cooperation scheme. Figure indicates example channel allocations across spreading codes and time. For user $k \in\{1,2, \ldots, K\}, \mathcal{D}(k)$ denotes the decoding set. The non-orthogonal spreading waveform of the $k$-th user is denoted by $s_{k}(t)$. Each user now transmits and receives simultaneously on different spreading codes during time Phase I. . . . . . . . . . . . . . . 
4.2 Outage probability performance (under high-SNR approximation) of spacetime coded scheme in an asynchronous underloaded CDMA uplink with $m=$ $K$ and diversity combining. The threshold spectral efficiency is $R=1 \mathrm{bit} / \mathrm{sec} / \mathrm{Hz}$. The point-to-point CDMA uplink performance $(m=1)$ is shown for comparison. The outage probability curves without high-SNR approximation (dashed lines) are also plotted for comparison. . . . . . . . . . . . . . . .

4.3 Outage probability performance (under high-SNR approximation) of spacetime coded scheme in an asynchronous overloaded CDMA uplink $(K>N)$ with $m=K$ and diversity combining. The threshold spectral efficiency is $R$ $=1 \mathrm{bit} / \mathrm{sec} / \mathrm{Hz}$. The point-to-point CDMA uplink performance $(m=1)$ is also shown for comparison. . . . . . . . . . . . . . . . . . .

4.4 Outage probability performance (under high-SNR approximation) of spacetime coded scheme in an asynchronous overloaded CDMA uplink $(K>N)$ with $m=N$ and diversity combining. The threshold spectral efficiency is $R$ $=1 \mathrm{bit} / \mathrm{sec} / \mathrm{Hz}$. The point-to-point CDMA uplink performance $(m=1)$ is also shown for comparison. . . . . . . . . . . . . . . . .

4.5 Conditional outage probability performance comparison of diversity combining and code combining schemes for fully-loaded CDMA system configuration with $m=K=N=8$. The outage probability is conditioned on $\boldsymbol{R}$. The threshold spectral efficiency is $R=1 \mathrm{bit} / \mathrm{sec} / \mathrm{Hz}$. Code combining is $0.01 \mathrm{~dB}$ better than the diversity combining and so the plots are almost indistinguishable. 71

4.6 Conditional outage probability performance comparison of fully-loaded CDMA system configuration ( $m=K=N=8$ ) under the constraint of uniform input probability (QPSK and 16-QAM modulation) and unconstrained Gaussian input. The outage probability is conditioned on $\boldsymbol{R}$. We assume diversity combining at the base station. The threshold spectral efficiency is $R=0.8$ bits/sec/Hz. . . . . . . . . . . . . . . . . . . . .

4.7 Block diagram of the receiver structure . . . . . . . . . . . . . .

4.8 BER and diversity performance of three-user DF sharing in an asynchronous uplink for various inter-user channel qualities. Performance of maximumratio-combining (MRC) with 1,2 and 3 antennas is plotted for comparison (dotted lines). . . . . . . . . . . . . . . . . . . .

5.1 Relay placement scenarios: (1) Array near destination (2) Array in the middle (3) Array near source (4) Equidistant clusters (5) serial equidistant placement between source and destination along $\mathrm{X}$ axis (6) serial equidistant placement between source and destination along $\mathrm{Y}$ axis (7) (sample) Uniform distribution of relays . . . . . . . . . . . . . . . . . . . . .

5.2 Outage probability performance with $K=8$ under various relay placement scenarios and different numbers of cooperation stages. . . . . . . . . . . . .

5.3 Outage probability performance with $K=8$ under various relay placement scenarios and different number of cooperation stages. . . . . . . . . . .

5.4 Outage probability performance comparison with $K=4, K=8$, and, $K=16$ and $T=2, \cdots, 6$ for Scenario $(5) \ldots \ldots \ldots \ldots$ 
5.5 Multistage cooperation gain for scenario (5) as a function of path loss coefficient $\alpha$ for $K=8, \mathrm{SNR}=15 \mathrm{~dB}$ and $R=1 \mathrm{bit} / \mathrm{sec} / \mathrm{Hz}$. . . . . . . . . .

5.6 Multistage cooperation gain for scenario (5) as a function of target rate $R$ for $K=8$ and $\mathrm{SNR}=15 \mathrm{~dB}$ and $\alpha=5$. Note that the multistage cooperation gain is plotted here for the case of large path loss. . . . . . . . . . . . . . 


\section{List of Tables}

4.1 Normalized (by the fraction of available degrees of freedom utilized by each cooperating user) transmit power and normalized (by the fraction of available degrees of freedom utilized by each user under noncooperative transmission) spectral efficiency parameterizations consistent with Laneman [1]. $r$ is the transmission rate in bits/sec and $W$ is the bandwidth in Hz. . . . . . . . . .

5.1 Optimal values of $T$ and cooperation gain at $15 \mathrm{~dB}$ SNR for different network topologies shown in Fig. $5.1 \ldots \ldots \ldots . . \ldots . . \ldots$ 


\section{Notation}

We use the following notation and symbols throughout this thesis.

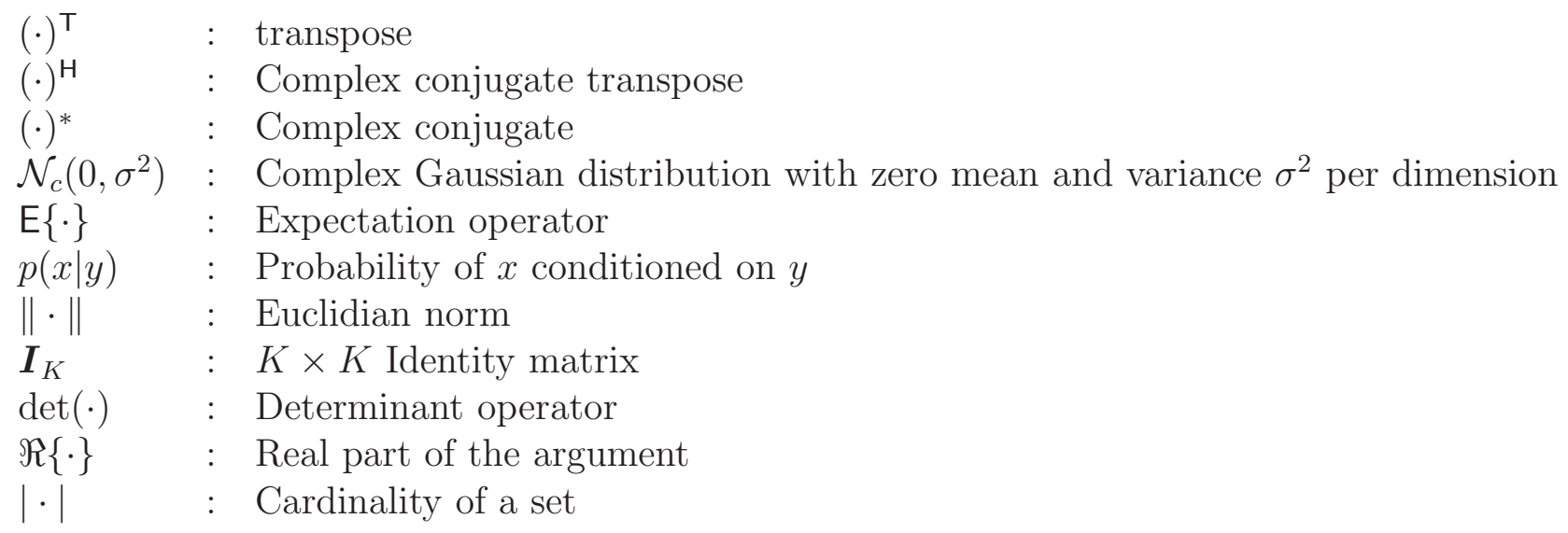

Bold uppercase letters denote matrices while bold lower case letters denote vectors. 


\section{Chapter 1}

\section{Introduction}

With the deployment of $3 \mathrm{G}$ broadband network services, users are enjoying the benefits of a wide variety of life enhancing and personalized services. The increasing use of wireless services such as mobile telephony, wireless internet, real-time video, and live TV over wireless channels, have sparked interests in the research community to explore potential $4 \mathrm{G}$ technologies that could serve the demands for even higher data rates and quality-of-service(QoS) requirements. $3 \mathrm{G}$ cellular networks provide ubiquitous connectivity but comparatively low data rates when compared to WLAN which can offer much higher data rates but only cover smaller geographic areas. Smart integration of these technologies might be a promising move for next generation wireless networks. Often wireless system designers are faced with number of challenges that include a complex time-varying nature of the wireless channel (fading and multipath), limited radio frequency spectrum, and interference between the signals transmitted by many simultaneous active users. Fading is the main channel impairment in the wireless communication systems and most of the research in wireless community is centered around investigating advanced signal transmission/reception methods to combat or exploit fading.

Fading is often mitigated by using diversity techniques, antenna diversity (spatial diversity) being attractive since it can employed independent of time and frequency diversity. In particular, transmit diversity can be generated by transmitting information using multiple antennas. At the receiver, the independently faded copies of the transmitted signal can be combined in an appropriate manner to better detect the signal of interest. The landmark 
contributions by Telatar [2] and Foschini [3] have shown that capacity of multiple antenna systems exceeds that of single antenna communication systems. The multiple antenna systems promise high capacity gains only when antenna elements experience uncorrelated fading channels. This requires the antenna spacing of about several wavelengths of the carrier frequency. But in many cases, it is not possible to implement multiple antennas at the mobile units with the desired spacing between them. For example, with $3 \mathrm{G}$ wireless systems using carrier frequencies of the order of $1 \mathrm{GHz}$, wavelength can not be much smaller than $0.3 \mathrm{~m}$. Due to physical size and hardware complexity constraints, implementation of a large number of antennas with many meters dimension is not practical in many situations, such as uplink of the cellular environment, WLANs, or sensor nodes in case of wireless sensor networks.

Recently, a promising method called user cooperative communications has been proposed that enables single antenna mobile users in a multiuser environment to share their antennas and additional resources to generate a virtual multiple antenna transmitter that allows them to achieve transmit diversity. The basic idea is the following. Each single antenna user in a multiuser communication environment cannot individually generate transmit diversity. However, due to the broadcast nature of the wireless medium, it may be possible for users to receive each other's transmission. The users can then forward a processed version of these received signals along with their own data. Each user's data gets forwarded from antennas of different users over statistically independent fading paths, thus yielding a transmit diversity effect. Cooperation between users leads to interesting trade-offs in terms of transmit power per user and data rates. One might guess that additional transmission resources, for example, power, time, or bandwidth will be needed in order for user to transmit its own data and also data for other users it is cooperating with, thereby increasing the total transmit power and lowering the data rates. However, each user will need less power due to diversity gains and also improve spectral efficiency due to cooperative diversity. It is not known a priori which of these effects will dominate the system performance. Hence the development on design and implementation of cooperation protocols will need a careful investigation of several complicated issues such as overall interference in the network, resource allocation algorithms, cooperation group assignment, power allocation between user's own data and cooperative data etc. 


\subsection{Dissertation Motivation and Outline}

Wireless mobile terminals usually suffer from a number of practical constraints such as average power constraints and bandwidth limitations. Designing an efficient system model under these constraints while meeting demands for higher data rates, increased user coverage, higher network capacity calls for investigation of innovative and practical techniques that improve spectral efficiency and link reliability in cooperative wireless networks. The issue that we try to address in this work is how efficiently the users or dedicated relays in wireless networks can share the transmission resources in a collaborative manner that would lead to increases in reliability, range, and data rate of wireless systems with single antenna transceivers. In particular, the work in this dissertation focuses on the following three areas of research.

- Joint power allocation and relay selection in cooperative wireless networks Most prior work on two-stage cooperative diversity either 1) makes no attempt to optimize power, or 2) optimizes power, assuming a cooperating group has been assigned a priori. There has been a very little work on joint relay selection and power allocation in multiuser decode-and-forward (DF) cooperative networks. To avoid an excessive power expenditure, while still allowing users to satisfy their target data rates, it is necessary to allocate the source power that selects the optimal decoding set and then optimally allocate the relay transmission powers. This kind of optimal power allocation leads to joint relay selection and power allocation problem which has been rarely considered in the literature on cooperative diversity. Note that in case of a DF protocol, the set of decoding relays is a function of both inter-user channels as well as the source power during the first phase of transmission. We develop a power minimization algorithm in a two-stage decode-and-forward cooperative diversity uplink such that each user satisfies its target data rate. We assume for the expositional simplicity that the users have been allocated orthogonal channels for transmission. Each user in the proposed system transmits its own data towards the base station and also serves as a relay for other users. The base station assigns one or more relays to each user in order to minimize total power in the uplink. The relay selection is based upon the instantaneous user 
to base station channels, inter-user channels and also the target rates of the users. The simulation results indicate significant power savings over a non-cooperative uplink, under proposed joint relay selection and power minimization algorithm in a DF cooperative uplink when using a space-time coded cooperative diversity.

- The impact of non-orthogonal channel allocation in cooperative wireless networks In conventional two-stage decode-and-forward cooperation schemes, the source broadcasts its message in the first time phase. All potential relays that can decode source's message transmit in the second time phase on orthogonal channels. The design and analysis of two-stage cooperative diversity protocols often relies on the assumption of orthogonal channel allocation between relays and source terminals and synchronous communication between the signals transmitted by different cooperating terminals in the networks which may lead to bandwidth inefficient system design. Both these assumptions may also require accurate coordination causing significant overhead in the network. Also, high bandwidth efficiency is one of the key requirements to achieve high target rates with limited spectrum availability. To deal with these problems, we propose a multiuser DF cooperative diversity protocol that operates in the presence of inter-user orthogonality and evaluate the outage probability performance of asynchronous CDMA uplink under fading channels. We analyze the information-outage probability performance of the proposed protocol in underloaded CDMA, fully-loaded CDMA and overloaded CDMA under diversity combining at the base station in the high-signal-to-noise ratio (SNR) regime.

- The performance of multistage cooperation

In a conventional dual-phase decode-and-forward cooperative diversity protocol, the transmission between source and the destination is accomplished in two orthogonal time phases. The source transmits in the first time phase and then cooperating terminals that can decode source's transmission may transmit during the second phase. These schemes have been shown to extract full diversity in the total number of terminals and not just the number of decoding terminals. However, in many scenarios of two-stage 
cooperation, not every relay takes part in cooperation because of not being able to decode source's transmission. Having noted that each relayed transmission leads to either an increase in the average SNR at the destination (as in the case of space-time coded cooperative diversity) or an increase in mutual information at the destination (as in incremental redundancy-based cooperative diversity protocols), it might seem intuitive to design a multistage cooperation scheme where most of the relays could contribute to the performance enhancement at the destination. In the multistage cooperation scheme that we propose, the transmission between source and destination takes place in $T \geq 2$ equal duration and orthogonal time phases. We consider a decode-and-forward cooperation where relays successfully decode the message before forwarding. In the first time phase, the source broadcasts its message which is received by all potential relays. During subsequent time phases, the relays that have successfully decoded the message using information from all previous transmitting relays, transmit a space-time code for the source's message. The non-decoding relays keep accumulating information and transmit in the later stages when they are able to decode the message. We develop and analyze the outage probability of multistage cooperation protocol under orthogonal relaying. Through analytical results, we obtain the near-optimal placement of relays that gives the best performance when compared with most other candidate relay location strategies of interest. For the relay placement scenario where the relays are placed along the straight line between source and destination in an equidistant manner, we also investigate an interesting tradeoff between an increased SNR and decreased spectral efficiency as the number of cooperation stages is increased.

The rest of the dissertation is organized as follows. In Chapter 2 we present background material required for the subsequent development of the work. In Chapter 3 we propose a strategy to minimize the total power in a decode-and-forward (DF) multi-user, multi-relay cooperative uplink, such that each user satisfies its quality-of-service (QoS) data rate. We model the total power minimization problem as an optimization problem where the objective function (total network power) is a convex function of user powers and the constraints are target rates of users which are concave functions. We then solve the optimization prob- 
lem by the Lagrange multiplier method. The solution to the optimization problem in DF cooperative uplink leads to an iterative algorithm that jointly performs relay selection for cooperation and optimally allocates source and the relay powers. Chapter 4 presents an outage probability analysis for a multi-user decode-and-forward (DF) cooperative diversity protocol that operates in an asynchronous CDMA uplink while relaxing the inter-user orthogonality constraint and also presents guidelines for a practical system design for asynchronous cooperative diversity. We develop and analyze exact outage probability expression for multistage cooperation protocol in Chapter 5. Chapter 6 concludes the dissertation where we also present future research ideas. 


\section{Chapter 2}

\section{Theoretical Background}

This chapter presents a variety of key concepts needed for the subsequent development of this dissertation. In particular, the chapter begins with a brief introduction to multipath fading, one of the key performance limiting factors in wireless networks. At the physical and medium access layers, there are two main issues in cellular communication: multiple access and interference management. We present the code-division multiple-access (CDMA) system where all users can transmit over the same frequency band simultaneously. The idea behind CDMA is to code user's information bits with a unique spreading sequence and at the receiver, retrieve user specific data with its assigned spreading code. In this system, the simultaneous transmissions by users cause interference. We discuss multiuser detectors that exploit the knowledge of multiple access interference in CDMA systems. The basic definitions from information theory including mutual information and outage probability that will be used quite often in the dissertation are then described. We conclude this chapter with a discussion on evolution of cooperative communication from point-to-point multiple-antenna communications and also point out the related work in this area.

\subsection{The Wireless Channel}

Once a signal is transmitted over a wireless medium, it undergoes reflection, refraction and defraction and arrives at the receiver via multiple paths. Fluctuations in the instantaneous received signal strength due to multipath propagation of the transmitted signal is 
termed as fading. Scattering and propagation over long distances increasingly attenuates signal power introducing an effect called path-loss. Due to basic propagation mechanisms, multipath components of a signal arrive at the receiving antenna with different propagation delays and amplitudes. The multipath copies of the transmitted signal might add constructively, thereby increasing the signal-to-noise ratio (SNR), or destructively, thereby decreasing the SNR. These SNR fluctuations cause fading. In order to model slow flat fading, often block fading channel model is used which was also introduced in [4]. The fading coefficients in block fading are modeled as constant over a block of symbols and vary independently between blocks. Suppose a block of symbols consists of $N$ symbols each with duration $T$. Then the baseband received signal in a frequency non-selective block fading channel can be expressed as

$$
r(t)=h s(t)+n(t), \quad 0 \leq t \leq N T
$$

where $s(t)$ is the transmitted signal, $h$ captures the effects of fading and $n(t)$ captures the effects of receiver thermal noise. There exists various channel models based on the probability distribution of the fading. Throughout this dissertation, we use Rayleigh fading channel model where we assume that there are large number of scatterers so that $h$ can be modeled as complex i.i.d Gaussian using central limit theorem.

\subsection{Multiuser Communications and CDMA}

With the increasing demands on higher data rates, it is a challenging task to accommodate a large number of mobile users in a finite radio spectrum. There are various multiple access techniques that allow multiple users to share the finite radio spectrum. For example, in case of time division multiple access (TDMA) technique, time is partitioned into different time slots which are then assigned to each user. The receiver can simply switch to appropriate time epochs to detect the transmitted signal. On the other hand, frequency division multiple access (FDMA) assigns different carrier frequencies to each user and band-pass filtering of the received signal is used to recover the information from the user of interest. In these techniques, users share the channel by occupying a given time or frequency slot. It is also 
possible to let users transmit signals that overlap both in time and frequency and still detect each user's signal at the destination. This can be accomplished by assigning each user a unique signature sequence, the technique being commonly known as code-division multiple access (CDMA). Under fading, where the channel may vary with time and frequency, CDMA turns out to be superior to other multiple access techniques, because in CDMA systems, the frequency selectivity of the radio channel can be averaged out [5].

CDMA is a spread spectrum technique in which the signal is expanded in bandwidth with the help of a code which is independent of data. Synchronized reception and despreading operation with the same code is used to recover the original data. The bandwidth expansion factor is called the processing gain. CDMA systems may be described as underloaded, fullyloaded, or overloaded. Underloaded CDMA systems arise when the total number of users is less than the processing gain $N$. Fully-loaded CDMA corresponds to the case wherein the number of users is equal to the processing gain. Overloaded CDMA system, in which the number of users is larger than the processing gain, is of interest when $N$ cannot be increased due to bandwidth constraints. Overloaded CDMA uses linearly dependent signature waveforms. CDMA offers other advantages over FDMA and TDMA like universal frequency reuse, soft handoff, soft (user) capacity limit on the system, RAKE receiver reception to constructively combine multipath components, inherent resistance to narrowband interference and has been widely accepted in 3G standards such as WCDMA and cdma2000. Since all user's signals overlap in time and frequency, it results in multiple-access interference. If orthogonal spreading codes are assigned to users, then in synchronous communication environments, all the multiple access interference vanishes. Suppose a signal is assumed to be band-limited with bandwidth $W$ and time-limited over the time interval $[0, T]$, then the number of orthogonal signature sequences (spreading codes) that can be designed is approximately equal to $2 W T$ [6]. However, the orthogonality between spreading codes may get destroyed due to the time-varying nature of the wireless channel. Relaxing the constraint of orthogonal spreading codes actually has some advantages: 1) Maintaining strict synchronization between users to preserve the orthogonality between them is not needed which makes the system design less complex, 2) This places no hard constraint on the number of users in a CDMA system and the number of simultaneous users is no longer constrained to $2 W T$. User capacity is in 
fact decided by the received signal-to-noise ratio, cross-correlation between user's spreading codes and the receiver design [6].

It is convenient for both analysis and implementation to convert the continuous-time signal model into an equivalent discrete-time model. An equivalency between two models (in terms of the information contained in these two models) is achieved if the discrete-time model produces sufficient statistics for decision making at the receiver. In the following, two approaches to obtaining a discrete-time signal model from its continuous counterpart are discussed with each of its advantages and limitations.

\subsubsection{Discrete-time Signal Model for Synchronous CDMA}

For a multiuser system with total $K$ active users, the continuous time received signal at the receiver is

$$
r(t)=\sum_{i=0}^{M-1} \sum_{k=1}^{K} b_{k}[i] \alpha_{k} s_{k}\left(t-i T_{s}\right)+n(t)
$$

where $M$ is the block length, $\alpha_{k}$ is the flat fading channel coefficient for the channel between $k$-th user and the base station, $b_{k}[i]$ is the $k$-th user's transmitted symbol, and $n(\cdot)$ is an additive white Gaussian noise process with variance $\frac{N_{0}}{2}$ per complex dimension. The spreading waveform assigned to user $k$ is of the form

$$
s_{k}(t)=\sum_{j=0}^{N-1} c_{k}[j] \psi\left(t-j T_{c}\right), \quad 0 \leq t \leq T_{s}
$$

where $c_{k}[j] \in\left\{-\frac{1}{\sqrt{N}}, \frac{1}{\sqrt{N}}\right\}$ is the $j$-th element of user $k$ 's spreading code, $T_{c} \triangleq T_{s} / N$ is the chip period, $N$ is the processing gain, $T_{s}$ is the symbol period, and $\psi(t)$ is a unit-energy chip waveform having support over $\left[0, T_{c}\right]$, i.e.,

$$
\psi(t)=\left\{\begin{array}{cl}
\frac{1}{\sqrt{T_{c}}} & 0 \leq t \leq T_{c} \\
0, & \text { otherwise }
\end{array}\right.
$$

Note that since the channel gain $\alpha_{k}$ and the spreading waveform $s_{k}(t)$ do not depend on the symbol index $i$ in this model, we are implicitly assuming that the channel is time-invariant and constant over the block of symbols (which is $M T_{s}$ symbol periods long) and that the user uses the same spreading waveform in each symbol interval. 


\section{Matched-filtering with respect to Spreading Waveform}

The first action at the receiver, irrespective of the detection method used, is matched filtering. The continuous-time received signal in (2.2) is correlated with the spreading waveform of each user. The filtered output corresponding to user $l$ during the $m$-th symbol interval is

$$
\begin{aligned}
r_{l}[m] & =\int_{m T_{s}}^{(m+1) T_{s}} r(t) s_{l}\left(t-m T_{s}\right) d t \\
& =\sum_{i=0}^{M-1} \sum_{k=1}^{K} b_{k}[i] \underbrace{\alpha_{k} \int_{m T_{s}}^{(m+1) T_{s}} s_{k}\left(t-i T_{s}\right) s_{l}\left(t-m T_{s}\right) d t}_{h_{k, l}}+n[m] .
\end{aligned}
$$

Note that the above integral is zero unless $i=m$ since the spreading waveform has a support over one symbol interval $T_{s}$. Stacking the matched filter outputs of each user, the discretetime system model in the matrix-vector model looks like [7]

$$
\boldsymbol{r}[m]=\boldsymbol{H} \boldsymbol{b}[m]+\boldsymbol{n}[m]
$$

where $\boldsymbol{r}[m] \triangleq\left[\begin{array}{llll}r_{1}[m] & r_{2}[m] & \cdots & r_{K}[m]\end{array}\right]^{\top}, \boldsymbol{b}[m] \triangleq\left[\begin{array}{llll}b_{1}[m] & b_{2}[m] & \cdots & b_{K}[m]\end{array}\right]^{\top}, \boldsymbol{H}$ is a function of channel parameters and the cross-correlations between spreading waveforms with $[\boldsymbol{H}]_{k, l} \triangleq \int_{-\infty}^{\infty} \alpha_{k} s_{k}(t) s_{l}(t) d t$, and $\boldsymbol{n} \sim \mathcal{N}(\mathbf{0}, \boldsymbol{R})$ is no longer a white noise with $[\boldsymbol{R}]_{k, l} \triangleq$ $\int_{-\infty}^{\infty} s_{k}(t) s_{l}(t) d t$. Note that if spreading codes are orthogonal, i.e., $\int_{-\infty}^{\infty} s_{k}(t) s_{l}(t) d t=\delta_{k, l}$, then $\boldsymbol{H}$ is diagonal and all multiple access interference vanishes. For synchronous CDMA, correlation of continuous-time received signal with the spreading waveform produces sufficient statistics for the detection/estimation of the originally transmitted signal. However, it requires the knowledge of spreading waveforms of each user at the receiver.

\section{Matched-filtering with respect to Chip Waveform}

Chip-matched filtering can also be used to arrive at the discrete-time signal model which is simpler in implementation and convenient for analysis, especially for asynchronous CDMA. The received signal when matched filtered at the chip level yeilds sufficient statistics in synchronous channels. The $q$-th matched filtered output during the $m$-th symbol interval is 


$$
\begin{aligned}
r[m, q] & =\int_{m T_{s}+q T_{c}}^{m T_{s}+(q+1) T_{c}} r(t) \psi\left(t-m T_{s}-q T_{c}\right) d t \\
& =\sum_{i=0}^{M-1} \sum_{k=1}^{K} b_{k}[i] \alpha_{k} \sum_{j=0}^{N-1} c_{k}[j] \int_{m T_{s}+q T_{c}}^{m T_{s}+(q+1) T_{c}} \psi\left(t-m T_{s}-q T_{c}\right) \psi\left(t-i T_{s}-j T_{c}\right) d t+n[m, q] \\
& =\sum_{k=1}^{K} \alpha_{k} b_{k}[m] c_{k}[q]+n[m, q] .
\end{aligned}
$$

Stacking chip-matched filtered outputs for one symbol period, the matrix-vector model is given by

$$
\begin{aligned}
\boldsymbol{r}[m] & =\underbrace{\boldsymbol{S} \boldsymbol{A}}_{\boldsymbol{H}} \boldsymbol{b}[m]+\boldsymbol{n}[m] \\
& =\boldsymbol{H} \boldsymbol{b}[m]+\boldsymbol{n}[m]
\end{aligned}
$$

where $[\boldsymbol{S}]_{:, k} \triangleq\left[c_{k}[0] c_{k}[1] \cdots, c_{k}[N-1]\right]^{\top}, \boldsymbol{b}[m] \triangleq\left[b_{1}[m] \quad b_{2}[m] \quad \cdots \quad b_{K}[m]\right]^{\top}, \boldsymbol{A}$ is a diagonal matrix; each diagonal entry representing the channel gain $\alpha_{k}, \boldsymbol{H}$ is thus a function of channel gains and spreading codes, and $\boldsymbol{n} \sim \mathcal{N}_{c}\left(0, N_{0} \boldsymbol{I}\right)$. It can be noticed that for synchronous communication, there is no inter-symbol interference (ISI), i.e., $k$-th user's matched-filtered output at time $i$ depends on the transmitted symbols from all users only at time $i$. This is also known as multiple-access interference (MAI).

\subsubsection{Discrete-time Signal Model for Asynchronous CDMA}

In practice, because of the transmitter asynchronism and channel parameters, the transmitted symbols from various users arrive at the receiver at different times. The received signal under the asynchronous criterion can be expressed as

$$
r(t)=\sum_{i=0}^{M-1} \sum_{k=1}^{K} b_{k}[i] \alpha_{k} s_{k}\left(t-i T_{s}-\tau_{k}\right)+n(t)
$$

where now $\tau_{k}$ represents the $k$-th user's propagation delay.

\section{Matched-filtering with respect to Delayed Spreading Waveforms}

At the receiver, the received signal is matched-filtered with respect to the delayed spreading waveform of each user. By Cameron-Martin formula [8], this process generates sufficient 
statistics, $r_{l}[m]$, given by

$$
\begin{aligned}
& r_{l}[m]=\int_{-\infty}^{\infty} r(t) \alpha_{l}^{*} s_{l}\left(t-\tau_{l}-m T_{s}\right) d t
\end{aligned}
$$

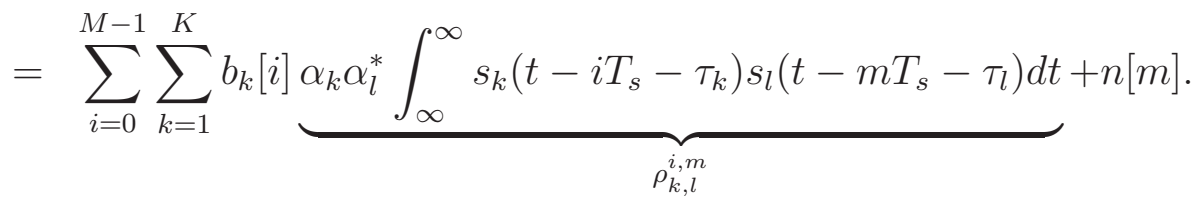

Stacking all matched-filtered outputs and dropping the time index from the model, results in

$$
r=H b+n
$$

where $\boldsymbol{r}=\left[r_{1}[m] r_{2}[m] \cdots r_{K}[m]\right]^{\top}, \boldsymbol{b}=[\boldsymbol{b}[0] \boldsymbol{b}[1] \cdots \boldsymbol{b}[M-1]]^{\top}$ with $\boldsymbol{b}[i]=\left[b_{1}[i] b_{2}[i] \cdots b_{K}[i]\right]^{\top}$, $\boldsymbol{H}$ is the cross-correlation matrix whose $\left(n, n^{\prime}\right)$-th element is given by $\rho_{k, l}^{i, m}$ with $n=i K+$ $(k-1)$, and $n^{\prime}=m K+(l-1)$, and $\boldsymbol{n} \sim \mathcal{N}_{c}\left(\mathbf{0}, N_{0} \boldsymbol{H}\right)$. It can be noted that though correlating the received waveform with the delayed spreading waveforms produces sufficient statistics in the discrete-time model, such correlation requires the knowledge of individual user's delays as well as their spreading waveforms. The discrete-time model can be formed without the knowledge of the delays of all users with chip-matched filtering as explained next which also results in a low complexity implementation.

\section{Matched-filtering with respect to Chip Waveform}

We consider a case where the continuous time received signal is sampled at the chip rate. Though simpler in implementation than correlation with delayed spreading waveforms, the process does not produce sufficient statistics in asynchronous channels. Because, the chip waveforms shifted in time by symbol intervals in this case represent a sub-optimum basis set for representing the signal in the context of the energy captured [9].

The $q$-th matched filtered output during the $m$-th symbol interval can be written as

$$
\begin{aligned}
r[m, q]= & \int_{m T_{s}+q T_{c}}^{m T_{s}+(q+1) T_{c}} r(t) \psi\left(t-m T_{s}-q T_{c}\right) d t \\
= & \sum_{i=0}^{M-1} \sum_{k=1}^{K} b_{k}[i] \alpha_{k} \sum_{j=0}^{N-1} c_{k}[j] \int_{m T_{s}+q T_{c}}^{m T_{s}+(q+1) T_{c}} \psi\left(t-m T_{s}-q T_{c}\right) \psi\left(t-i T_{s}-j T_{c}-\tau_{k}\right) d t \\
& \quad+n[m, q] .
\end{aligned}
$$


Denote the maximum delay as

$$
l=\max _{k} l_{k} \quad \text { and } \quad l_{k}=\left\lceil\frac{\tau_{k}+T_{c}}{T_{s}}\right\rceil .
$$

Assuming delays uniformly distributed between $\left[0, T_{s}\right]$, and after some manipulations [7],

$$
r[m, q]=\sum_{i=0}^{l} \sum_{k=1}^{K} b_{k}[m-i] \underbrace{\alpha_{k} \sum_{j=0}^{N-1} \int_{0}^{T_{c}} \psi(t) \psi\left(t-j T_{c}-\tau_{k}+m T_{s}+q T_{c}\right)}_{h_{k}[i, q]} d t+n[m, q] .
$$

Again, after some manipulations, we stack match-filtered outputs of each user for one symbol period, then we stack $m$ successive samples of received vectors ( $m$ being the smoothing factor as discussed in [7]) and arrive at the following linear model

$$
\boldsymbol{r}=\boldsymbol{H} \boldsymbol{b}+\boldsymbol{n} .
$$

The matched filtered output for user $k$ at time $i$ may now be affected by the symbols transmitted by all other users during previous time interval and also by the $k$-th user's symbols from previous symbol intervals. Thus, under asynchronous communication scenario, both ISI and MAI are present in the received signal. ISI may be due to the asynchronous transmissions between users or the multipath nature of the channel. MAI is due to the underlined CDMA technique [10]. Because of the ISI, the detection problem in an asynchronous channel is more complicated that in synchronous channels. The detection problem in synchronous channel can only focus on one symbol interval while detection problem in asychronous channels should take into account thee symbols decisions on adjacent overlapping symbols of each user.

Chip-matched filtering even in the case of asynchronous CDMA channels has been considered widely in the literature. The statistics obtained by this criterion, though not sufficient, gives a reasonably accurate discrete-time model [9], particularly when macthed-filtered output is oversampled to minimize the inferential loss.

\subsubsection{Multiuser Detection}

Multiple-access interference which is the main concern in case of CDMA can be mitigated by the use of multiuser detectors. The conventional detector as will be presented in this 
section, detects each user independent of others. Unlike conventional detector, multiuser detectors use the information about multiple users jointly to better detect individual user [6]. Hence the design of multiuser detectors is an important research area in multiuser communication systems.

\section{Optimal Multiuser Detection}

Unless $\boldsymbol{H}$ is diagonal, user's symbols interfere with each other leading to multiple-access interference. Under the assumption of channel state information (CSI) at the receiver, the choice of $\boldsymbol{b}$ that reduces the probability of bit vector error satisfies

$$
\hat{\boldsymbol{b}}=\arg \min _{\boldsymbol{b}}\|\boldsymbol{r}-\boldsymbol{H} \boldsymbol{b}\|^{2}
$$

It is optimum in the sense of minimizing the probability of error. Though this structure yeilds globally optimal solution, the complexity of this type of receiver structure is prohibitively high. The complexity increases in an exponential manner with the number of users and the block length [6]. We will look at various suboptimal multiuser detectors that are easier to implement.

\section{Linear Multiuser Detection Techniques}

In this case, symbol-by-symbol decisions are made on the statistics obtained after applying a linear filter to the received signal vector. That is

$$
\boldsymbol{d}=\Omega^{\mathrm{H}} \boldsymbol{r}
$$

where $\Omega$ is a linear filter whose coefficients are obtained according to some optimization criterion [11].

- (Conventional) Matched Filter Detector: The decision statistic is obtained as

$$
\boldsymbol{d}=\boldsymbol{H}^{\mathrm{H}} \boldsymbol{r}
$$

Matched filter detector is easy to implement. This detector neglects the MAI from other users. The performance of matched filter detector (conventional detector) depends 
highly on the cross-correlation properties of the spreading codes and the performance is poor unless the spreading code cross-correlations are very small. The correlation with all other users gives rise to MAI which has a significant impact on the performance of conventional detector. This type of detector also suffers from the near-far problem in that due to geographical locations, stronger users (most probably which are closer to the destination) may dominate the weaker users. The advantage of this detector is that its implementation requires only the knowledge of the spreading waveform of the user being demodulated. Decorrelating and MMSE multiuser detector are the most popular linear multiuser detectors which are briefly discussed in the following sections.

- Decorrelating Multiuser Detector: Decorrelating multiuser detector is the optimal detector in that it corresponds to the maximum likelihood detector when the received amplitudes of all users are unknown. The first step in implementing a decorrelator is matched filtering with respect to the channel matrix. Starting with the general discrete-time received signal model

$$
\boldsymbol{r}=\boldsymbol{H} \boldsymbol{b}+\boldsymbol{n} .
$$

After premultiplying the received signal by $\boldsymbol{H}^{\mathrm{H}}$,

$$
\begin{aligned}
\boldsymbol{y} & =\boldsymbol{H}^{\mathrm{H}} \boldsymbol{r} \\
& =\underbrace{\boldsymbol{H}^{\mathrm{H}} \boldsymbol{H}}_{\boldsymbol{R}} \boldsymbol{b}+\boldsymbol{H}^{\mathrm{H}} \boldsymbol{n} .
\end{aligned}
$$

Decision statistic is then obtained as

$$
\begin{aligned}
\boldsymbol{d} & =\boldsymbol{R}^{-1} \boldsymbol{y} \\
& =\boldsymbol{b}+\underbrace{\boldsymbol{R}^{-1} \boldsymbol{H}^{\mathrm{H}} \boldsymbol{n}}_{\boldsymbol{v}}
\end{aligned}
$$

where effective noise $\boldsymbol{v}$ is no longer white and is distributed as $\boldsymbol{v} \sim \mathcal{N}_{c}\left(\mathbf{0}, N_{0} \boldsymbol{R}^{-1}\right)$. The power of the noise increases as the cross-correlation between spreading codes gets large. It can be seen that the decorrelating multiuser detector removes any multipleaccess interference at the expense of enhancing the background noise. Despite the noise enhancement, the decorrelator improves the performance over matched filter detection. 
It also provides optimal near-far resistance. Though the computational complexity is lower than that of optimal multiuser detector, the computations needed to invert $\boldsymbol{R}$ matrix are difficult to perform in practice. This type of detector also requires knowledge of all user's signature waveforms to demodulate any given user.

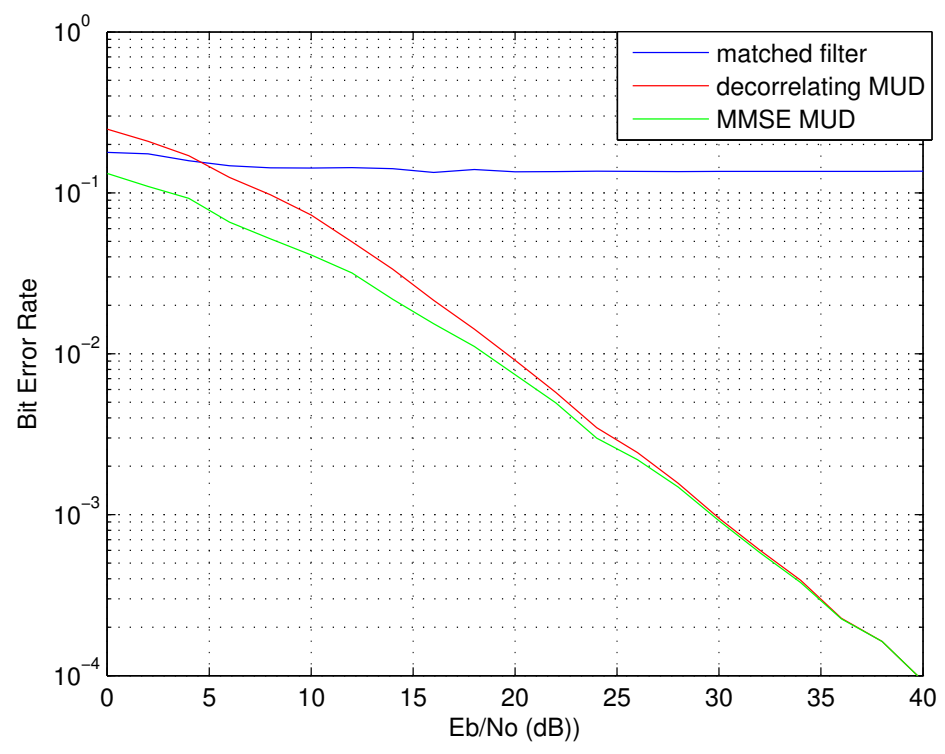

Figure 2.1: Bit error rate performance of a synchronous CDMA in a Rayleigh flat fading channels for chip-matched filter detector, decorrelating multiuser detector (MUD) and MMSE MUD. Total number of users is 14, processing gain is 15 and random spreading codes are used.

- MMSE Multiuser Detector : As the name suggests, the minimum mean-squared (MMSE) multiuser detector minimizes the mean square error between the decision statistics and the symbols we wish to demodulate. The linear filter that minimizes the mean square error can be written as

$$
\hat{\Omega}=\arg \min _{\boldsymbol{\Omega}} E\left\{\left|\boldsymbol{b}-\Omega^{\mathrm{H}} \boldsymbol{r}\right|\right\}
$$

Expanding the expectation and using gradient techniques, the linear filter is obtained as

$$
\hat{\boldsymbol{\Omega}}=\left(\boldsymbol{H} \boldsymbol{H}^{\mathrm{H}}+N_{0} \boldsymbol{I}\right)^{-1} \boldsymbol{H} .
$$

The performance of MMSE detector is usually superior to the decorrelator because it exploits the knowledge of both the MAI and also the noise. At high SNR, MMSE 
and decorrelator have the same performance. We note however that, MMSE requires the knowledge of background noise while decorrelator does not. MMSE balances the effects of MAI and noise while decorrelator completely eliminates MAI at the expense of increased noise. It also offers a significant practical advantage that it can be adapted blindly or with the use of training sequence.

\subsection{Information Theory for Wireless Fading Channels}

Information theory has evolved ever since the admittance of Shannon's landmark paper [12] which focused on various elements of information theory including channel capacity. Over the last decade, an increase in the capacity promised by multi-antenna communication techniques have stimulated many information theoretic analyses. Furthermore, information theory has been used as a design tool to optimize the signal transmission and reception strategies at the transceiver side. Analysis using information theory provides theoretical bounds on the system performance and in many cases also provides guidelines toward the practical system design. The results thus obtained by using mathematical tools from information theory provide a baseline for comparison with practical systems. In this dissertation, we widely make use of mutual information and outage probability as the performance evaluation tools. Hence, we provide an overview of some of the concepts from information theory.

\subsubsection{Entropy (Self Information)}

Entropy is the amount of uncertainty contained in a random variable. Consider a continuous random variable $X$. The differential entropy of a random variable $X$ is given by

$$
H(X)=-\int_{x} p(x) \log p(x) d x
$$

where $p(x)$ is the probability density function of $X$. The entropy of discrete random variables sets up a lower bound on the average description length of the random variable and hence is widely used as a performance measure in data compression. 


\subsubsection{Mutual Information}

Mutual information represents reduction in the uncertainty of random variable $X$ after observing possibly dependent random variable $Y$. The mutual information between two continuous random variables $X$ and $Y$ is given by

$$
\begin{aligned}
I(X ; Y) & =H(X)-H(X \mid Y) \\
& =\int_{x} \int_{y} p(x, y) \log \frac{p(x, y)}{p(x) p(y)} d x d y .
\end{aligned}
$$

The above definition indicates that if $X$ and $Y$ are independent, the mutual information is zero, since knowledge of $Y$ does not reduce any uncertainty about $X$.

\subsubsection{Unconstrained Channel Capacity and Outage Probability}

For a communication channel with input $X$ and output $Y$, the theoretical capacity in Shannon sense is defined as

$$
C=\max _{p(x)} I(X ; Y)
$$

where the maximization is with respect to the input distribution. Capacity is the mutual information maximized over all possible input distributions. It indicates the maximum rate at which information can be transmitted across the channel and still be recovered at the destination with very low error probability.

Capacity as can be seen is primarily a mathematical concept. However, the reason capacity has had operational relevance in the design of communication systems is because of the emerging technical advances that make the design of capacity approaching error correcting codes a reality. Furthermore, the delay and complexity needed to approach true Shannon-sense capacity have turned out to be reasonable in current technology.

\section{The AWGN Channel}

Consider a communication channel where the transmitted symbols are corrupted by the additive white Gaussian noise (AWGN) channel. Let $X$ be the real (1-dimensional) input to 
the channel with the power constraint $P$, i.e., $\mathrm{E}\left\{X^{2}\right\}=P$. The output of an AWGN channel is

$$
Y=X+N
$$

where $N$ is a Gaussian random variable distributed as $N \sim \mathcal{N}\left(0, N_{0}\right)$ and $N$ is independent of the signal $X$. The mutual information between the channel input and output is then

$$
\begin{aligned}
I(X ; Y) & =H(Y)-H(Y \mid X) \\
& =H(Y)-H(X+N \mid X) \\
& =H(Y)-H(N \mid X) \\
& =H(Y)-H(N) .
\end{aligned}
$$

The last equality follows from the independence of $X$ and $N$. Since $N$ is Gaussian, $H(N)=$ $\frac{1}{2} \log _{2} 2 \pi e N_{0}$ and $\mathrm{E}\left\{Y^{2}\right\}=P+N_{0}$. Then, the entropy of $Y$ is bounded by $\frac{1}{2} \log _{2} 2 \pi e\left(P+N_{0}\right)$. Therefore, the mutual information can also be bounded as [13]

$$
\left.I(X ; Y) \leq \frac{1}{2} \log _{2} 2 \pi e\left(P+N_{0}\right)\right)-\frac{1}{2} \log _{2} 2 \pi e N_{0},
$$

with equality when $X$ is Gaussian with the same variance $P$. Hence, the information capacity of the Gaussian channel which is the mutual information maximized over all possible input distribution is

$$
\begin{aligned}
C & =\max _{p(x)} I(X, Y) \\
& =\frac{1}{2} \log _{2}\left(1+\frac{P}{N_{0}}\right) \text { bits/transmission. }
\end{aligned}
$$

For a bandlimited channel with bandwidth $W$, and input signal transmitted over the time interval $[0, T]$, the transmitted signal can be reconstructed by samples taken at a sampling rate $\frac{1}{2 W}$. In this case, the power per sample is $P T / 2 W T=P / 2 W$ and the noise power per sample is $N_{0} / 2$. The capacity per sample is then

$$
C=\frac{1}{2} \log _{2}\left(1+\frac{P}{N_{0} W}\right) \text { bits/sample. }
$$

Since there are $2 W$ samples per second, the capacity is

$$
C=W \log _{2}\left(1+\frac{P}{N_{0} W}\right) \text { bits/sec. }
$$


Equation (2.30) gives the highest rate in bits per transmission (channel use) at which information can be transmitted over the noisy channel with arbitrarily low probability of error. Under the assumptions of random codes with very long codeword lengths, Shannon proved that there exists a code that can achieve the data rate very close to channel capacity with extremely low probability of error and it is not possible to operate at higher rates than the capacity in which case the error probability is bounded away from zero.

\section{Ergodic Fading Channels}

Ergodic nature of the channel arises in wireless fading channels. The fading channel obeys large and small-scale fading, where the latter can be categorized as flat/frequencyselective and slow/fast fading and is observed in both temporal and spatial domain. It is very difficult to come up with an exact physical model of the time-varying random channel and hence statistical models are often used to describe the statistical nature of the channel, the most common being Rayleigh fading channel model. Let $x$ be the input with average power $P$. In case of Rayleigh fading channel, the observed signal can be written as

$$
y=h x+n
$$

where $h$ is a zero mean, unit variance complex Gaussian random variable representing the effect of small-scale fading. The envelope of $h$ then follows Rayleigh distribution. Conditioned on $h$, the channel is AWGN with instantaneous SNR $=|h|^{2} P / N_{0} W$. Each realization of the fading channel has a maximum information rate associated with it. The conditional or instantaneous channel capacity in this case is

$$
C_{\text {instantaneous }}=\log \left(1+\frac{|h|^{2} P}{N_{0} W}\right)
$$

In analyzing the capacity of fading channels, two commonly used statistics are the ergodic capacity and the outage capacity or outage probability. Ergodic capacity of the fading channel in Shannon sense can be obtained as

$$
C_{\text {ergodic }}=\mathrm{E}_{h}\left\{\log \left(1+\frac{|h|^{2} P}{N_{0} W}\right)\right\}
$$


To achieve ergodic capacity, the codeword length should be long enough to observe all channel states during the codeword duration. Since $\log (\cdot)$ is a concave function, by Jensen's inequality,

$$
\mathrm{E}_{h}\left\{\log \left(1+\frac{|h|^{2} P}{N_{0} W}\right)\right\} \leq \log \left(1+\frac{\mathrm{E}_{h}\left\{|h|^{2}\right\} P}{N_{0} W}\right) .
$$

Thus ergodic capacity of a fading channel when only receiver has channel state information (CSI) is less than the capacity of AWGN channel with same average SNR and hence fading reduces the channel capacity when only receiver CSI is available.

\section{Non-ergodic Fading Channels}

For delay-limited applications or slowly fading channels, the realized channel SNR may not support rate $R$ and hence the notion of ergodic channel capacity does not exist. This is because, under the conditions of no CSI at the transmitter side, the transmitter fixes a certain SNR independent of the instantaneous received SNR and encodes a code with rate $R$. The channel realizations for which $\log \left(1+\frac{|h|^{2} P}{N_{0} W}\right) \geq R$, information can be sent over the channel with very small probability of error. On the other hand, whenever the instantaneous channel capacity $\log \left(1+\frac{|h|^{2} P}{N_{0} W}\right)<R$, information cannot be conveyed reliably over the channel. This event corresponds to an outage event and the corresponding probability is called outage probability. It is defined as [14]

$$
\mathrm{P}_{\mathrm{out}}=\operatorname{Pr}\left\{\log \left(1+\frac{|h|^{2} P}{N_{0} W}\right)<R\right\} .
$$

For Rayleigh fading channel, the received signal is

$$
r=h x+n
$$

where $h \sim \mathcal{N}_{c}(0,1)$ and $n \sim \mathcal{N}_{c}\left(0, N_{0}\right)$. Let average SNR be denoted by SNR $=\frac{P}{N_{0} W}$, then outage probability can be evaluated as

$$
\begin{aligned}
\mathrm{P}_{\text {out }} & =\operatorname{Pr}\left\{\log \left(1+|h|^{2} \mathrm{SNR}\right)<R\right\} \\
& =\operatorname{Pr}\left\{|h|^{2}<\frac{2^{R}-1}{\mathrm{SNR}}\right\} \\
& =1-\exp \left(-\frac{2^{R}-1}{\mathrm{SNR}}\right) .
\end{aligned}
$$




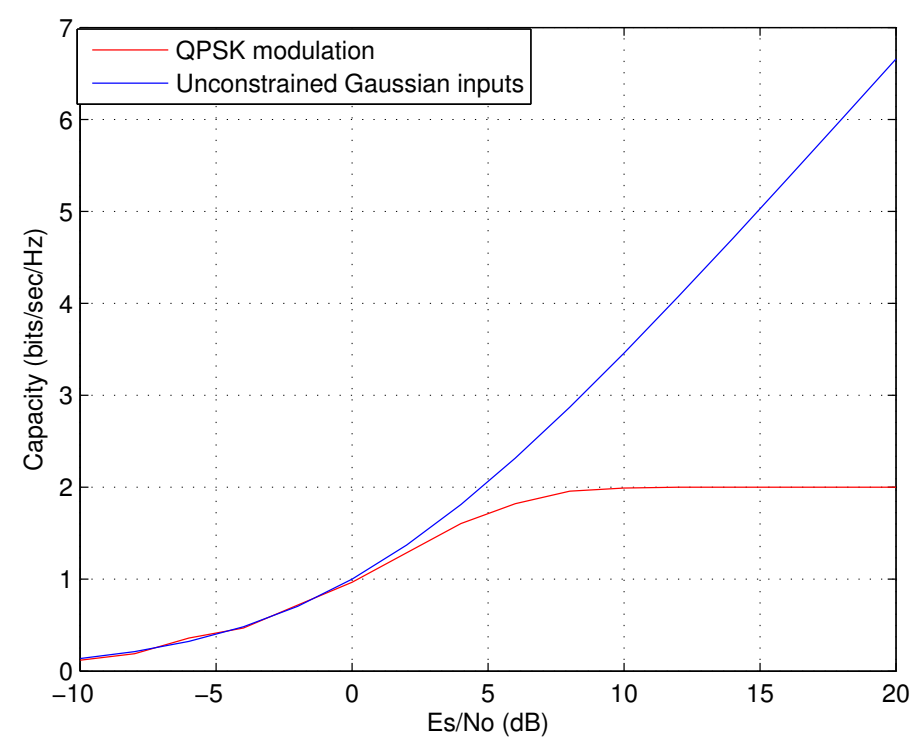

Figure 2.2: Capacity of QPSK modulation and the unconstrained Shannon capacity in AWGN channel

Outage probability acts as a lower bound on the codeword error probability of practically coded systems.

\subsubsection{Capacity Under Modulation Constraints}

As seen from the previous section, for Gaussian channels, the capacity is achieved when using Gaussian distributed input symbols. However, in practice, input symbols are often chosen from a finite signal set $\mathcal{S}$, (for example, BPSK, QPSK, QAM modulation) and usually with equal probability. In that case, the channel capacity is nothing but mutual information between channel input and output given by (2.25) with $p(x)$ decided by the underlined modulation signaling set $\mathcal{S}$. The mutual information under modulation-constrained input 
for the received signal model in (2.38) can be rewritten as [15]

$$
\begin{aligned}
C_{\text {constrained }} & =\mathrm{E}_{x, y, h}\left[\log \frac{p(x, y)}{p(x) p(y)}\right] \\
& =\mathrm{E}_{x, y, h}\left[\log \frac{p(y \mid x)}{p(y)}\right] \\
& =\mathrm{E}_{x, y, h}\left[\log \frac{p(y \mid x)}{\sum_{x \in \mathcal{S}} p(y \mid x) p(x)}\right] \\
& =m-\mathrm{E}_{x, y, h}\left[\log \frac{\sum_{x \in \mathcal{S}} p(y \mid x)}{p(y \mid x)}\right]
\end{aligned}
$$

where $m=\log _{2} M$, M being the signal constellation size for a specific modulation. As an example, the capacity of AWGN channel under QPSK modulation is shown in Fig. 2.2. The unconstrained capacity with Gaussian inputs is also shown for comparison. It can be seen that the capacity under modulation constraints is inferior to the unconstrained capacity at high SNRs. In addition, at high SNR, the capacity under modulation constraints saturates at $\log _{2} M$ bits/sec/Hz while the unconstrained capacity increases linearly with SNR.

\subsection{Spatial Diversity}

For point-to-point communication systems with Rayleigh fading, where there exists only a single communication path between the transmitter and a receiver, the probability of deep fade is significantly high. It is observed that by providing a receiver with multiple copies of the same transmitted signal through multiple independent faded channels (also called diversity branches), the probability of deep fades can be significantly reduced. Diversity essentially reduces the dependence of the actual received signal-to-noise ratio (SNR) on the instantaneous channel state and is often indicated by the asymptotic slope of the error rate curves when plotted against signal-to-noise ratio (SNR) in decibels. Various methods to achieve diversity exist including time diversity, frequency diversity, and spatial diversity. Time diversity adds redundant data bits in the time domain. The time intervals during which redundant information is sent exceed the coherence time of the channel providing multiple independent faded copies of the signal to the receiver. Channel coding may be viewed as a form of time diversity. In frequency diversity method, the information symbols are sent over multiple carrier frequencies. Here, the carrier frequencies are separated by 
at least the coherence bandwidth of the channel and hence experience independent fades. An example of frequency diversity is multicarrier modulation. The RAKE receiver is a recent implementation of frequency diversity for CDMA. RAKE receiver provides diversity gains only when there exist multiple resolvable paths. Spatial diversity (also known as antenna diversity) is based on reception or transmission of wireless signals via multiple antenna elements along with appropriate signal processing that combines the signals from the various antennas. The diversity benefits with time or frequency diversity techniques are usually obtained at the expense of increase in either delay or bandwidth. Spatial diversity achieved using multiple antennas at the transmitter and/or receiver side (also known as antenna diversity) is an attractive alternative since it does not sacrifice time or bandwidth while providing increased received SNR.

\subsubsection{Multi-antenna Systems and Space-Time Communications}

The use of multiple antennas at the transmitter and receiver in wireless systems, popularly known as MIMO (multiple-input multiple-output) systems, has gained much fame over the last decade due to its powerful performance-enhancing capabilities. Much of the initial excitement about multiple antenna systems was due to the pioneering work by Telater [2] and Foschini [3] suggesting notable capacity gains over conventional single antenna systems under the rich scattering channel model.

Considering a transmitter with $M_{T}$ transmit antennas and a receiver with $M_{R}$ receive antennas, the channel can be represented by the $M_{R} \times M_{T}$ matrix $\boldsymbol{H}$. The entries $h_{i, j}$ of $\boldsymbol{H}$ represent the channel gains from transmit antenna $j$ to receive antenna $i$. The $M_{R} \times 1$ received signal $\boldsymbol{y}$ is

$$
\boldsymbol{y}=\sqrt{\frac{P}{M_{T}}} \boldsymbol{H} \boldsymbol{s}+\boldsymbol{n}
$$

where $\boldsymbol{s}$ is the $M_{T} \times 1$ transmit symbol vector, $\boldsymbol{n}$ is the $M_{R} \times 1$ additive white complex Gaussian noise vector with $\mathrm{E}\left(\boldsymbol{n} \boldsymbol{n}^{\mathrm{H}}\right)=N_{0} \boldsymbol{I}_{M_{R}}$ and $P$ is the total energy available at the transmitter over a symbol period. Let's assume that the fading coefficients in $\boldsymbol{H}$ are i.i.d. complex Gaussian random variables. 


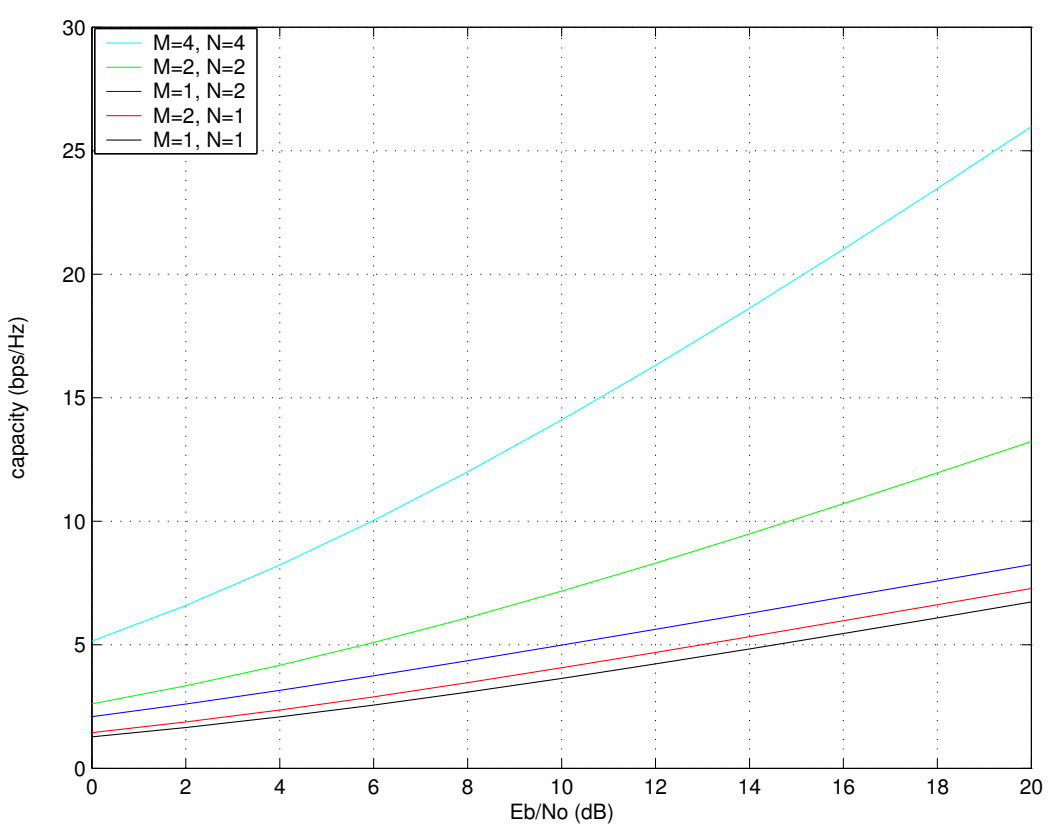

Figure 2.3: Ergodic capacity of MIMO system with different antenna configurations

Under the assumption of channel knowledge only at the receiver, the ergodic capacity is given by [16]

$$
C=\mathrm{E}_{\boldsymbol{H}}\left[\log \operatorname{det}\left(I_{M_{R}}+\frac{\mathrm{SNR}}{M_{T}} \boldsymbol{H} \boldsymbol{H}^{\mathrm{H}}\right)\right]
$$

where SNR $=\frac{P}{N_{0}}$.

Fig. 2.3 gives the ergodic capacity curves as a function of SNR for different MIMO antenna configurations. We note that the capacity of SIMO channel is higher than MISO channel. It can also be shown from equation (2.42) that in the high-SNR regime, when $M_{T}$ and $M_{R}$ are fixed, capacity grows by $\min \left(M_{T}, M_{R}\right)$ for each additional $3 \mathrm{~dB}$ increase in SNR. Also, with increase in transmit antennas $M_{T}$, while keeping $M_{R}$ fixed, the capacity is bounded and converges to $M_{R} \log _{2}(1+\mathrm{SNR})$. If the number of receive antennas increases while keeping $M_{T}$ fixed, the capacity increases as $\log _{2} M_{R}$. With simultaneous increase in the number of transmit and receive antennas, the capacity increases linearly with $\min \left(M_{T}, M_{R}\right)$ $[17]$.

As seen above, MIMO systems offer advantages over single-input single-output (SISO) channels in terms of multiplexing gains (which improve spectral efficiency) under the assumption of uncorrelated fading between the paths created by multiple antennas [2, 18]. 
Bell Labs Space Time Architecture (BLAST) which was first proposed by Foschini [3] can be used to achieve capacity for an arbitrary number of transmit and receive antennas. In this method, independent symbol streams are transmitted from each transmit antenna. The receiver incorporating multiple receive antennas then processes these symbol streams using appropriate detection algorithm. Although the number of independent data streams transmitted per channel use is very important factor in MIMO communications, from an engineering perspective, the link efficiency characterized by both the number of transmitted data streams as well as the bit-error-rate (BER) plays significant role in the performance evaluation of such systems. It was observed that multiple antennas can also offer diversity gains, improving the BER. Space time coding (STC) techniques using multiple transmit antennas target on achieving high diversity gains. In the space time coding technique [19, 20, 21, 22], the same information is transmitted from different antennas in an appropriate manner to improve the reliability of the system. To summarize, the goal of the BLAST system is to achieve multiplexing gain. BLAST system transmits $M$ symbols/channel use (assuming $M$ transmit antennas) to achieve this goal. Whereas STC tries to achieve maximum diversity gain. Thus STC transmits $M \leq 1$ reliable symbol/channel use. The key feature of spacetime coding is to provide diversity with very low encoding/decoding complexity. There exists an optimal diversity-multiplexing tradeoff which sets a fundamental performance limit of a MIMO channel system [23]. The diversity-multiplexing tradeoff is essentially the tradeoff between bit-error-rate and the data rate in a channel. The main result in [23] says that given a MIMO channel, it is possible to simultaneously extract both diversity gains and capacity gains, however there exists a fundamental trade-off between how much of any type of gain any coding scheme can obtain: higher capacity gain comes at the price of sacrificing diversity. Although multiple-antenna techniques improve the capacity and BER performance, it is not always possible to incorporate multiple antennas at the wireless terminals due to size and complexity constraints. To obtain the gains promised by multiple antenna communication systems using single antenna wireless terminals, a new arena of cooperative wireless communications has been evolved. 


\subsubsection{Cooperative Communications}

The genesis of cooperative communication can be traced back to the work on relay channel [24]. The simplest relay channel model consists of a source, relay and the destination. The noisy version of the information sent by the source is received by both the relay and the destination. In order to help destination decode source's transmission, the relay forwards a processed version of the received signal to the destination. Of many other forwarding strategies in cooperative wireless communications, the two most favored strategies are decode-and-forward (DF) cooperation and amplify-and-forward (AF) cooperation. In decode-and-forward cooperation, the relay first fully decodes the source transmission. Upon successful decoding, it might repeat the information or might transmit additional parity bits for the original codeword. Amplify-and-forward cooperation consists of the relay amplifying the noisy received signal from the source and retransmitting it to the destination. Although the noise of the partner is amplified in this scheme, the destination still receives two independently-faded versions of the signal and is thus able to make better decisions for the transmitted symbols [25]. In this dissertation, we limit our attention to decode-and-forward cooperation. User cooperation, a simple twist on relaying differs from a simple relay channel in that users in the wireless network assist in relaying each other's information while still being able to send their own data. Cooperative communication is thus a new form of spatial diversity that eliminates the need for incorporating multiple antennas into a single mobile unit while still achieving the transmit diversity gains via cooperation of multiple users in the system. The broadcast nature (by which transmitted signal is received by multiple nodes) of the wireless medium and its ability to achieve diversity through independent paths are the two key features which contribute to the cooperative communication.

There has been a great deal of research effort in finding the capacity of relay networks and developing efficient user cooperative protocols. Cover and El Gamal [24] developed upper and lower bounds on the capacity of non-fading relay channel and found that the relay channel capacity is bounded by the minimum of the rates of transmission of the constituent broadcast and multiple access channels. Høst Madsen [26] developed an alternative capacity results for the relay channel with the restriction that source and the relay do not trans- 
mit simultaneously in the same frequency band. As an extension to single-relay channels, various multiple relay network models have been studied in the literature. [27, 28, 29, 30]. Sendonaris et al. [31] were the first to consider information theoretic framework of twouser cooperation where they analyze achievable rate regions and outage probability under multiple-access channel model. The authors also discuss CDMA implementation for this two-user cooperation scheme [32]. Laneman et al. provided outage probability analysis for multi-user cooperative diversity using optimal space-time codes [1]. The authors in [33, 34] introduced the concept of coded cooperation where each user instead of repeating the source information transmits incremental redundancy for its partner upon successfully decoding the partner's data. More advanced issues of cooperative wireless networks such as relay selection, grouping and power allocation have also been addressed by numerous authors, for example, see $[35,36,37,38]$.

\section{Orthogonal Relaying Protocols}

We briefly review two candidate two-stage DF protocols first analyzed in [1] that assume orthogonality between relayed transmissions and equal power allocation between the source and other relays. In our work, we separately investigate the impact of optimal power allocation and non-orthogonal relaying on the performance of these protocols and therefore use the candidate protocols as a baseline for comparison. Consider $K$ users communicating with a single destination on orthogonal channels (perhaps using orthogonal frequency slots or orthogonal spreading codes in case of CDMA). Each user has its own data to send and may also act as a relay for other users. The channel allocation for direct transmission, incrementalbased cooperative diversity and space-time coded cooperative diversity is depicted in figures 2.4-2.6.

- Incremental Redundancy-Based Cooperative Diversity:

The source $k$ starts broadcasting its transmission to the destination. The other users that can decode source's transmission participate in cooperation and form a decoding set $\mathcal{D}(k)$. The users in $\mathcal{D}(k)$ then transmit additional parity bits (incremental redundancy) for the source's transmission on orthogonal subchannels. Under code 


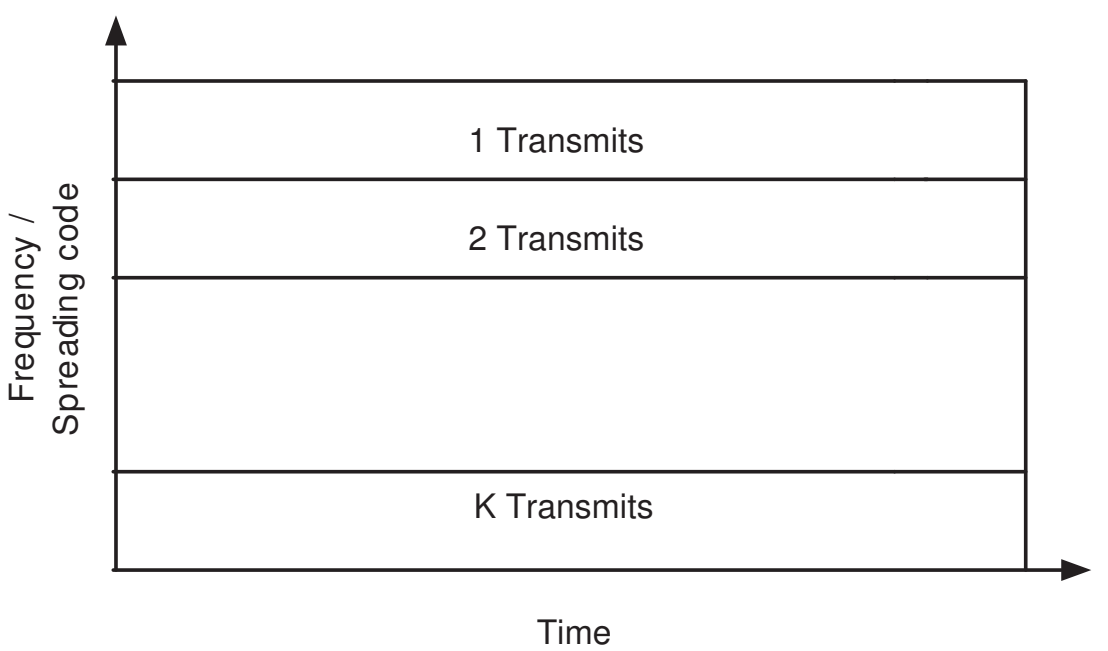

Figure 2.4: Channel allocation for the non-cooperative system.

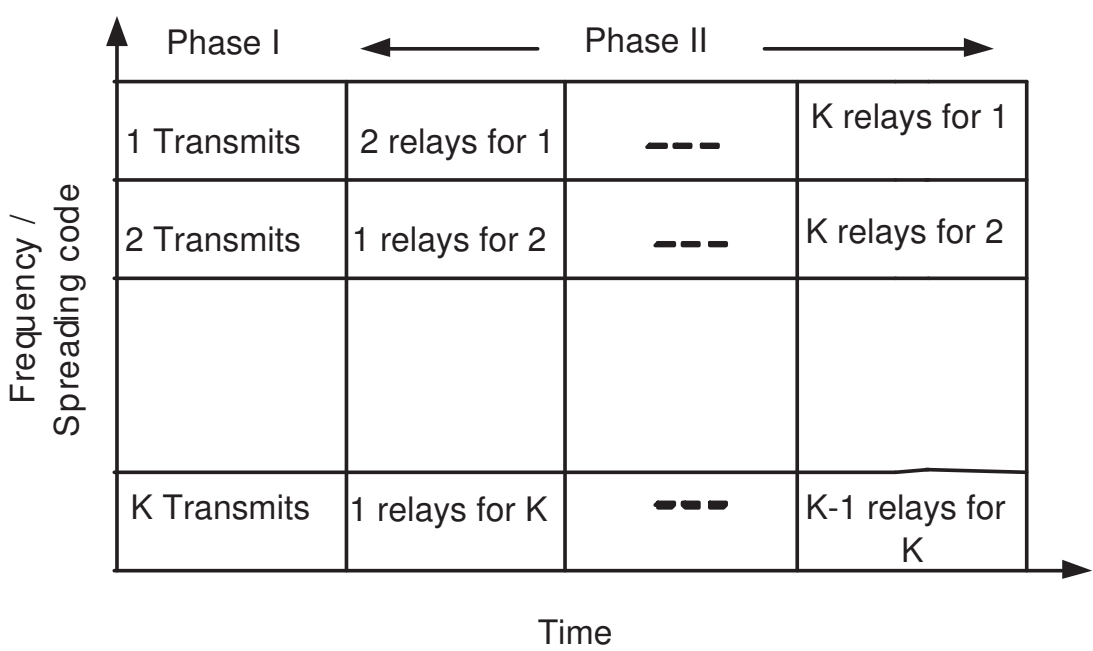

Figure 2.5: Channel allocation for incremental-based cooperative diversity.

combining, the destination receives the original codeword from the source and also the parity bits from relays which are combined in a suitable manner for detection. The mutual information for the channel between the $k$-th user and the destination under code combining is

$$
I_{\mathrm{rep}, \mathrm{cc}}=\frac{1}{K} \log \left(1+\mathrm{SNR}\left|h_{s, d}\right|^{2}\right)+\frac{1}{K} \sum_{r \in \mathcal{D}(s)} \log \left(1+\mathrm{SNR}\left|h_{r, d}\right|^{2}\right)
$$

where SNR $\triangleq \frac{K P_{c}}{N_{0} W}$ is the average SNR normalized by $K . \quad P_{c}, N_{0}$, and $W$ denote the continuous time transmit power per terminal, noise density and total bandwidth 


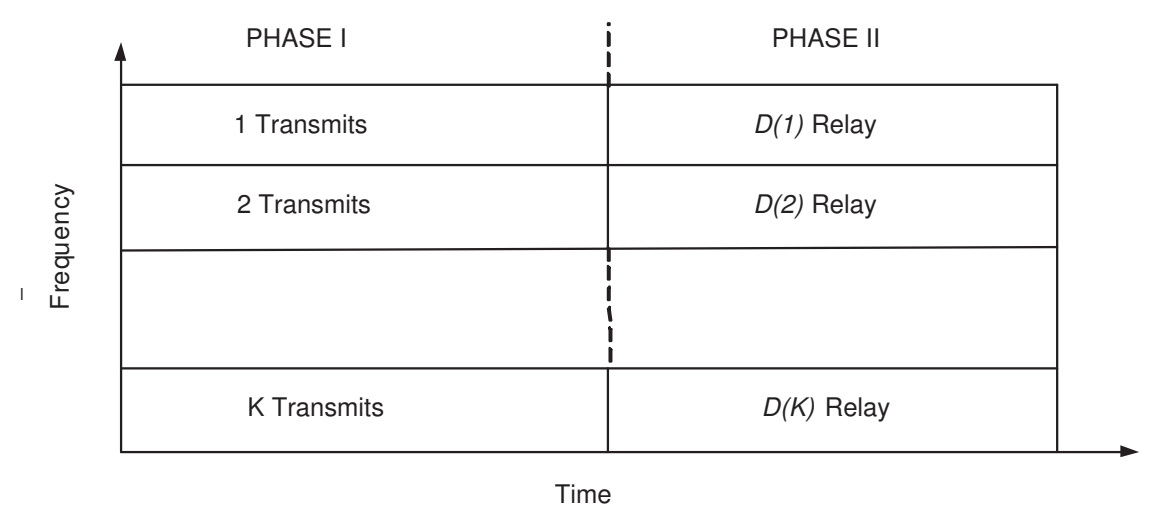

Figure 2.6: Channel allocation for space-time coded cooperative diversity.

respectively. The factor $K$ appears in front of the log term and also as a normalization factor for the SNR term since the total transmission makes use of $K$ resources. The normalization of SNR and mutual information by this factor leads to a fair comparison with direct transmission (no cooperation scenario) since the cooperative system is inherently allocated more resources in terms of time duration or bandwidth.

- Space-Time Coded Cooperative Diversity

Instead of allowing each potential relay to transmit on a separate orthogonal subchannel, relays could collectively use a space-time code and transmit simultaneously on the same subchannel without interference, at least in theory. In synchronous systems, this can be implemented via orthogonal space-time block codes. Conditioned on the decoding set, the mutual information for the channel between the $k$-th user and the destination under diversity combining is

$$
I_{s t c}=\frac{1}{2} \log \left(1+\frac{2}{K} \mathrm{SNR}\left|\mathrm{a}_{s, d}\right|^{2}\right)+\frac{1}{2} \log \left(1+\frac{2}{K} \mathrm{SNR} \sum_{r \in \mathcal{D}(s)}\left|\mathrm{a}_{r, d}\right|^{2}\right) .
$$

The factor $1 / 2$ above is due to the time-phase orthogonality.

It was indicated in [1] that the incremental redundancy-based user cooperation becomes bandwidth inefficient with increase in the number of users since each user requires a separate subchannel for cooperation. Space-time coded cooperative diversity however is bandwidth efficient and its performance improves with increase in the number of 
users as cooperating users transmit over the same subchannel simultaneously using a (ideal) space-time code. The above mentioned classic protocols, however, place strict constraints on the synchronization between transmitting users, orthogonality between the channels allocated to cooperating users and do not place any constraints on the topology of the network. 


\section{Chapter 3}

\section{Joint Power Allocation and Relay Selection for Orthogonal Multiuser Cooperation}

The main assumption in cooperative communications is that relay transmissions are always beneficial, compared to direct transmission from source to destination. However, a relay might have extremely poor wireless channel conditions toward the destination during a specific time interval, and forwarding source's data through it would be harmful and wasteful in terms of transmission power. At a different time instance, the same relay might become useful if the wireless channels towards destination are strong (this could be possible when terminals are mobile). It is therefore imperative to develop cooperation schemes that determine which of the available relays are indeed useful, depending on their wireless channel conditions, as a function of time [37]. We propose in this work, an algorithm that performs relay selection while minimizing the total transmit power and satisfying certain quality-ofservice (QoS) requirement in multi-user, multi-relay decode-and-forward (DF) cooperative networks. Each user in the proposed system transmits its own data towards the base station and also serves as a relay for other users. The base station assigns one or more relays to each user in order to minimize total power in the uplink. The relay selection is based upon the instantaneous user to base station channels, inter-user channels and also the target rates of the users. The simulation results indicate significant power savings over a non-cooperative 
uplink, under proposed joint relay selection and power minimization algorithm in a DF cooperative uplink when using a space-time coded cooperative diversity.

\subsection{Introduction}

Recently, [1], [31] indicated performance improvements due to the use of cooperative diversity relative to point-to-point links in wireless networks when using single antenna at mobile nodes and equal power allocation. Later, efforts were made to further improve the performance of cooperative diversity by optimal power allocation and optimal group assignment. For example, considering a three terminal DF relay terminal network, optimal power allocation was studied when optimizing either achievable rates [39], outage events [40], or outage probability [41]. Using outage probability as an optimization criterion and total power constraint, the authors in [42] optimally allocated relay powers in DF multi-hop wireless networks. The power control algorithm, which attempts to minimize the outage probability under short-term and long-term total power constraints, was studied in [43] for two-user cooperation scheme, while [44] considered optimization of high-SNR approximations of outage probability for the multi-user space-time coded DF protocol. In [45], an opportunistic DF protocol was developed where a relay terminal is utilized depending on the overall network state with dynamic allocation of time and power. The above work demonstrated a significant performance improvement due to optimal power allocation over equal power allocation in cooperative networks. The partner choice problem in a DF cooperative network was investigated in [36], where authors devised a method to choose a single partner among available partners to increase the user cooperation gain. Grouping schemes for regenerative cooperative network of $N$ nodes, based on both centralized and distributed control strategies were presented in [35]. Bletsas et el., [37] proposed the best relay selection method that takes into account the instantaneous channel conditions of both source to relay and relay to destination channels.

Most previous work on cooperative diversity either 1) makes no attempt to optimize power, or 2) optimizes power, assuming a cooperating group has been assigned a priori. There has been very little work on joint relay selection and power allocation in multi-user 
cooperative networks. Power allocation for space-time coded DF cooperative diversity was studied in [46], where the authors presented a suboptimal solution to minimizing the outage probability where the source power is fixed (perhaps fixing the decoding set in effect) and the remaining power is equally distributed among the relays. The sub-optimal source power is obtained numerically through exhaustive search. Relative to [15], the novelty of our approach is the non-suboptimal solution for the source and relay powers and joint relay selection as explained below.

We consider a user-cooperative uplink where users have been allocated orthogonal channels for transmission (using, for example, orthogonal CDMA spreading codes ${ }^{1}$ ). Each user has its own data to send to the base station. We develop in this chapter, a strategy to minimize the total transmit power in a decode-and-forward (DF) user cooperative uplink, such that each user satisfies its quality-of-service (QoS), data rate. We model the total power minimization problem as an optimization problem where the objective function (total network power) is a convex function of user powers and the constraints are target rates of users which are concave functions. We then solve the optimization problem by Lagrange multiplier method. The solution to the optimization problem in DF cooperative uplink leads to an iterative algorithm that jointly performs relay selection for cooperation and optimally allocates source and the relay powers.

The remainder of the chapter is organized as follows. Section 3.2 introduces the uplink system model, channel model, and also describes the user cooperation protocol. Section 3.3 considers power consumption under non-cooperative uplink as a baseline for the proposed power minimization algorithm. Section 3.4 describes the proposed joint relay and power allocation algorithm under both diversity combining and code combining. Simulation results are presented in Section 3.5 and Section 3.6 concludes.

\footnotetext{
${ }^{1}$ Non-orthogonal codes can be dealt with using decorrelating multiuser detection and an additional noise variance factor [47].
} 


\subsection{System Model}

The uplink consists of $K$ users that have been allocated orthogonal channels. Each user has its own data to transmit to the base station, potentially using other users as relays. We consider a two-stage decode-and-forward (DF) protocol that consists of two transmission phases. During the first phase, each user $k$ broadcasts its message to the base station with power $p_{k}$. In the second phase, other users that can decode the $k$-th user's transmission form a decoding set $\mathcal{D}(k)$ and may serve as relays. Based on our proposed relay selection criterion, some relays from the decoding set would, however, remain silent even if they can decode the $k$-th user's transmission in order to reduce the power consumption in the network. Since each user acts as a source during the first time phase and may serve as relay during second time phase, we use the terms user, source and relay interchangeably. We consider two practical scenarios. In the first case, selected relays could transmit using incremental redundancy which leads to code combining of the relayed information. As an alternative, the selected relays may use a distributed space-time code for the source's transmission that leads to diversity combining of the relayed transmissions [1]. Incremental redundancy type of cooperation protocol enjoys full spatial diversity gains but at the expense of bandwidth inefficiency since each user requires a separate orthogonal channel for its own transmission and for relaying other user's data. More bandwidth efficient space-time coded cooperation provides full spatial diversity gains, however, requires symbol level inter-user synchronization.

The base station is assumed to have the knowledge of all instantaneous channel conditions including user to base station channels as well as inter-user channels and makes all assignment decisions $^{2}$. It then conveys the relay assignment and the optimized powers to users through a low rate feedback channel. The user-to-base station channels and the inter-user channels undergo independent quasi-static Rayleigh fading and path loss. The inter-user channels are non-symmetric, i.e., the channel between user $i$ and $j$ is, in general, different from the channel between user $j$ and $i$. All channels are statistically modeled as zero-mean, circularly symmetric complex Gaussian random variables. We model distance dependent path loss

\footnotetext{
${ }^{2}$ The assumption on the knowledge of all instantaneous channel gains at the base station is not practical. However, the results provide baseline for comparison with practical systems and also provide guidance in the design of practical systems.
} 
without shadowing. The channel (distance) between the $k$-th user and the base station is denoted by $h_{k, d}\left(d_{k, d}\right)$ while $h_{i, j}\left(d_{i, j}\right)$ denotes the inter-user channel (distance) between users $i$ and $j$. Let $g_{i, j}$ denote the channel gain for the link between users $i$ and $j$ where $g_{i, j}=\left|h_{i, j}\right|^{2} / d_{i, j}^{\alpha}, \alpha$ being the path loss coefficient.

\subsection{Power Consumption in a Non-cooperative Uplink}

For certain traffic types, e.g., real-time video, it is necessary that the transmission meets certain QoS requirement, e.g., data rate. The total network power consumption under a non-cooperative scenario, where users expend power to achieve target rates, can be found in a straightforward manner as follows.

The signal received by the base station $d$ due to the $k$-th user's transmission is

$$
r_{d}[n]=\sqrt{p_{k}} \frac{h_{k, d}}{d_{k, d}^{\alpha}} x_{k}[n]+v[n]
$$

where $p_{k}$ is the power used by the $k$-th source and $v[n]$ is the receiver noise and is distributed as $v[n] \sim \mathcal{N}_{c}(0,1)$. The mutual information for the channel between the $k$-th user and the base station is

$$
\log \left(1+p_{k} g_{k, d}\right) .
$$

The minimum transmit power required at the source $k$ to achieve the target rate $R$, under no cooperation, is

$$
p_{k, \mathrm{nc}}=\frac{2^{R}-1}{g_{k, d}} .
$$

The total uplink power under no cooperation scenario is

$$
p_{\mathrm{nc}}=\sum_{k=1}^{K} p_{k, \mathrm{nc}} .
$$

\subsection{Power Minimization in a User-Cooperative Uplink}

In wireless networks, at any given time instant, users might experience very different fading channel conditions. Users experiencing deep fades will then have to expend large 
amount of power in order to meet the QoS constraints as can be seen from (3.3). Spatial diversity created due to user cooperation might reduce the probability of deep fades, thus reducing the total transmit power. To further enhance the performance of user-cooperative uplink, it is imperative to devise algorithms for optimal relay selection and power allocation across source and relay terminals. Optimal power allocation in a DF uplink is complicated by the fact that decoding set is a function of both inter-user channels as well as the source power in the first phase. Given all instantaneous channel conditions, one of the naive approaches to the relay selection would be to perform exhaustive search over all possible decoding sets for a particular source by appropriately setting source power in the first phase and then deciding upon the decoding set that minimizes the total uplink transmit power after optimal power allocation. This relay selection process would alone require $\sum_{i=1}^{K-1}\left(\begin{array}{c}K-1 \\ i\end{array}\right)$ iterations per user, which is impractical for larger number of uplink users.

We develop an iterative algorithm that jointly performs relay selection for the purposes of cooperation and optimally allocates source and the relay powers. This iterative method requires only up to $K$ iterations. In this section, we state the proposed power minimization problem with respect to general mutual information expressions. In the later sections, we consider specific cooperative diversity protocols along with corresponding mutual information expressions and then provide solution to the joint relay selection and power minimization 
problem. The proposed power minimization problem can be formulated as follows.

$$
\operatorname{minimize} \sum_{k=1}^{K}\left\{p_{k}+\sum_{\substack{r=1 \\ r \neq k}}^{K} p_{k, r}\right\}
$$

subject to

$$
\begin{aligned}
& I_{1}=R_{1} \\
& I_{2}=R_{2} \\
& \quad \vdots \\
& I_{K}=R_{K} \\
& p_{k} \geq p_{k, \min } ; \quad k=1,2, \cdots, K \\
& p_{k, r} \geq 0 ; \quad k=1, \cdots, K ; \\
&
\end{aligned}
$$

where $p_{k}$ is the $k$-th user's transmit power during phase I, $p_{k, r}$ is the transmit power by relay $r$ when forwarding $k$-th user's message, $I_{k}$ is the mutual information for the channel between user $k$ and the base station which will be defined in subsequent sections, $R_{k}$ is the target rate of user $k$, and $p_{k \text {,min }}$ is the minimum power that source $k$ transmits with during the first time phase that helps choose relays. The power $p_{k, \text { min }}$ is updated in each iteration of the proposed iterative power minimization algorithm in order to select the most efficient (optimal) set of relays from the decoding set for source's transmission. The role of $p_{k \text {,min }}$ will be clarified further in the next Section. In the discussion to follow, we assume for the sake of exposition that the target rates of all users are the same, i.e., $R_{1}=R_{2}=\cdots R$.

In the above optimization problem, the minimization is done over source and the relay powers. The objective function here is a affine function of source and relay powers and hence is a convex function. Each constraint function, i.e., the target rate is a concave function. The convex optimization problem can now be solved by the Lagrange multiplier technique [48]. The solution to the above problem when selecting the optimal set of relays from a decoding set leads to an iterative algorithm as will be explained in the sequel. 


\subsubsection{Power Minimization under Code Combining}

In this section, we consider the power minimization algorithm for the incremental redundancy type coded cooperation protocol that was discussed in [1]. During the first time phase, each user $k$ transmits to the base station. During the second time phase, each relay selected after executing the proposed power minimization algorithm transmits the source's information over orthogonal subchannels. Each relay has it's own unique codebook for its own data and for each potential source's data. As an example, each relay could transmit a different part of the codeword which results in a code combining at the base station. Similar to the non-cooperative case, the mutual information due to the $k$-th user's transmission during the first phase is

$$
\frac{1}{K} \log \left(1+p_{k} g_{k, d}\right)
$$

The factor $1 / K$ is due to the fact that each source transmits during $1 / K$ of total time slots in incremental redundancy-based coded cooperative diversity [1]. A potential relay will be able to decode $k$-th user's message if the realized mutual information between user $k$ and the relay $r$ is greater than the fixed target spectral efficiency $R$. Therefore, the relay will be in the decoding set of user $k$ if

$$
\frac{1}{K} \log \left(1+p_{k} g_{k, r}\right) \geq R
$$

i.e.,

$$
p_{k} \geq \frac{2^{K R}-1}{g_{k, r}} .
$$

We denote by $p_{k, r, \text { min }}$, the minimum power source $k$ should transmit with that will guarantee successful decoding at the relay $r$. Hence

$$
p_{k, r, \min }=\frac{2^{K R}-1}{g_{k, r}} .
$$

The overall average mutual information between user $k$ and the base station under code combining is ${ }^{3}$

\footnotetext{
${ }^{3}$ Although it appears that spectral efficiency under incremental redundancy cooperative diversity goes to zero as $K$ goes to infinity, following the technique in [49] it can be easily shown that it approaches a fixed non-zero value.
} 


$$
\begin{aligned}
I_{k, c c} & =\frac{1}{K} \log \left(1+p_{k} g_{k, d}\right) \\
& +\frac{1}{K} \sum_{\substack{r=1 \\
r \neq k}}^{K} \underline{1}_{p_{k}>p_{k, r \min }} \log \left(1+p_{k, r} g_{r, d}\right)
\end{aligned}
$$

where $\underline{1}_{x>y}$ is a indicator function

$$
\underline{1}_{x>y}= \begin{cases}1, & \text { if } x>y \\ 0, & \text { otherwise. }\end{cases}
$$

Now with mutual information defined for the special case of code combining, we return to the optimization problem in (3.5). The Lagrangian equation for the optimization problem in $(3.5)$ is

$$
\sum_{k=1}^{K}\left\{p_{k}+\sum_{\substack{r=1 \\ r \neq k}}^{K} p_{k, r}\right\}-\sum_{i=1}^{K} \lambda_{i}\left(I_{i, c c}-R_{i}\right)=0 .
$$

By taking the derivative of (3.12) with respect to the source and relay powers, applying the Kuhn-Tucker conditions, and taking into account the non-negativity constraints,

$$
\begin{array}{r}
p_{k}=\max \left(\frac{\lambda_{k}}{\log 2}-\frac{1}{g_{k, d}}, p_{k, \min }\right) ; k=1, \cdots, K \\
p_{k, r}=\underline{1}_{p_{k}>p_{k, r \min }} \times \max \left(\frac{\lambda_{k}}{\log 2}-\frac{1}{g_{r, d}}, 0\right) \\
\quad \text { for } k=1, \cdots, K ; r=1, \cdots, K ; r \neq k
\end{array}
$$

where the powers $p_{k, \text { min }}$ in the first iteration are set according to the following rule:

$$
p_{k, \text { min }}= \begin{cases}p_{k, \mathrm{nc}}, & \text { if } p_{k, r, \text { min }} \geq p_{k, \mathrm{nc}}, \forall r ; \\ \arg \max _{r} \Omega, & \text { otherwise }\end{cases}
$$


where $\Omega=\left\{p_{k, r, \min } \mid p_{k, r, \text { min }} \leq p_{k, \text { nc }}\right\}$. Equation (3.15) has the following interpretation. During the first iteration, if the source to destination channel is stronger than any of the source to (potential) relay channels, then only direct transmission is preferred, else the source transmits with a minimum power that guarantees largest possible decoding set for its transmission. Hence, at the start of the first iteration of the algorithm, all potential relays for the source $k$ for which $p_{k, \text { min }} \geq p_{k, r, \text { min }}$ are the decoding relays. $\lambda_{k}$ is found by substituting source powers $p_{k}$ and the relay powers $p_{k, r}$ from (3.13) and (3.14) in the $k$-th constraint of (3.5) and solving the transcendental equation in $\lambda_{k}$. The source and relay powers are then obtained by substituting for $\lambda_{k}$ in (3.13) and (3.14). From the set of decoding relays considered during the previous iteration, the relays that resulted in corresponding $p_{k, r}=0$ after power minimization, are excluded from a set of decoding relays. This is because for any $p_{k, r}=0$, the resulting power minimization suggests not selecting that particular relay for cooperation purposes. The corresponding minimum source power to have second largest possible decoding set is then updated using equation (3.15) as also the source and relay powers. A total of up to $K$ iterations are needed to find the most efficient set of relays and the corresponding relay powers for each source. If the computed transmit powers do not change between successive iterations, the iterative procedure described in the proposed algorithm can be stopped. In a conventional DF protocol using constant power allocation, the relays remain silent if they cannot decode the source's transmission. However, in the proposed setup, whenever it is advantageous for the source to utilize a relay, it transmits with a sufficient power level that guarantees successful decoding at the relay. This also helps in finding the optimal source power for the first phase of transmission.

\subsubsection{Power Minimization under Diversity Combining}

We consider here the power minimization algorithm in the context of space-time coded protocol where during the second time phase of cooperation, the selected relays from the decoding set of a particular user use an ideal space-time code and hence can transmit simultaneously on the same subchannel [1]. Using (3.5), we develop a iterative relay selection and power minimization algorithm very similar to the code combining case discussed earlier. 
The mutual information due to $k$-th user's transmission during the first phase is

$$
\frac{1}{2} \log \left(1+p_{k} g_{k, d}\right)
$$

The factor $1 / 2$ is due to the time phase orthogonality in space-time coded protocol. A potential relay will be able to decode $k$-th users message if the realized mutual information between user $k$ and the relay $r$ is greater than the fixed spectral efficiency $R$. Therefore, relay will be in the decoding set of user $k$ if

$$
\frac{1}{2} \log \left(1+p_{k} g_{k, r}\right) \geq R
$$

i.e.,

$$
p_{k} \geq \frac{2^{2 R}-1}{g_{k, r}}
$$

Hence

$$
p_{k, r, \min }=\frac{2^{2 R}-1}{g_{k, r}}
$$

The overall average mutual information between user $k$ and the base station under diversity combining is

$$
\begin{aligned}
I_{k, s c} & =\frac{1}{2} \log \left(1+p_{k} g_{k, d}\right) \\
& +\frac{1}{2} \log \left(1+\sum_{\substack{r=1 \\
r \neq k}}^{K} \underline{1}_{p_{k}>p_{k, r \min }} p_{k, r} g_{r, d}\right)
\end{aligned}
$$

The diversity combining case with space-time coded protocol thus differs from the code combining case in the bandwidth utilization factor in front of the $\log$ terms which is $1 / K$ for the incremental redundancy based coded cooperative diversity and $1 / 2$ for the space-time coded cooperative diversity. It also differs in the mutual information expressions in that incremental redundancy based cooperative diversity with code combining involves sum-log expression while space-time coded diversity with diversity combining involves log-sum expressions for the second phase of transmission. The Lagrangian equation for the optimization 
problem in $(3.5)$ is

$$
\sum_{k=1}^{K}\left\{p_{k}+\sum_{\substack{r=1 \\ r \neq k}}^{K} p_{k, r}\right\}-\sum_{i=1}^{K} \lambda_{i}\left(I_{i, s c}-R_{i}\right)=0
$$

By taking the derivative of (3.21) with respect to the source and relay powers, applying the Kuhn-Tucker conditions and taking into account the non-negativity constraints,

$$
\begin{aligned}
& p_{k}=\max \left(\frac{\lambda_{k}}{\log 2}-\frac{1}{g_{k, d}}, p_{k, \min }\right) ; k=1, \cdots, K \\
& p_{k, r}=\max \left(\frac{\lambda_{k}}{\log 2}-\frac{1}{g_{r, d}}-\sum_{\substack{i=1 \\
i \neq r}}^{K} \underline{p}_{p_{k}>p_{k, i \min }} \times \frac{p_{k, i} g_{k, i}}{g_{r, d}}, 0\right) \\
& \quad \text { for } k=1, \cdots, K ; r=1, \cdots, K ; r \neq k .
\end{aligned}
$$

As seen from (3.23), the relay powers are now interdependent. The relay powers $p_{k, r}$ are found sequentially and in an iterative fashion, where sequence order is not crucial to finding the optimum solution. The relay powers are initially set to zero and are updated as the sequence of relay power equations in (3.23) is traced, for a particular value of $\lambda_{k}$. By varying the value of $\lambda_{k}$, a transcendental equation in $\lambda_{k}$ is solved. The remaining iterative steps of the underlined joint relay selection and power allocation algorithm remain the same as the code combining case.

\subsection{Simulation Results}

We assume for the simulation purposes that the users are distributed uniformly over a grid of $1 \times 1$ units with the base station located at position (1,1). All channels including the inter-user channels and the user-to-base station channels are independent. The channel coefficients are complex Gaussian with zero mean and unit variance. The path loss coefficient $\alpha$ is set to 3 . 


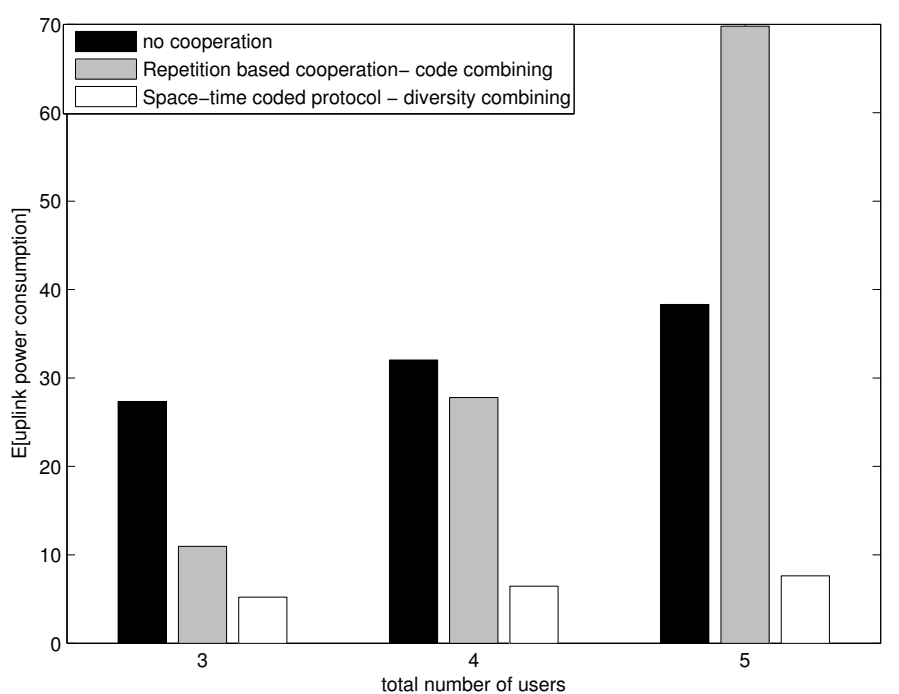

Figure 3.1: Average power consumption in a uplink as a function of total number of users. The target rate is $R=1 \mathrm{bit} / \mathrm{sec} / \mathrm{Hz}$. We see that incremental redundancy based cooperation improves performance up to about 5 users, while space-time cooperation uniformly outperforms no cooperation or incremental redundancy based cooperation.

Fig. 3.1 indicates the average power consumption in a uplink with respect to total number of users under direct transmission and two different user cooperation scenarios. The target rate is $R=1 \mathrm{bit} / \mathrm{sec} / \mathrm{Hz}$. It is seen that for a fixed rate, as we increase the total number of uplink users, the average total uplink power consumption under incremental redundancybased cooperative diversity with code combining exceeds that of direct transmission. This is because, under incremental redundancy-based cooperation, each relay requires a separate orthogonal channel for cooperation. With large number of users, this protocol becomes bandwidth inefficient and bandwidth inefficiency problem dominates any cooperation gain obtained. We see that incremental redundancy-based cooperation improves performance up to about 5 users. The space-time coded protocol with diversity combining performs uniformly better than no cooperation and incremental redundancy-based cooperation with code combining. This is because, with an increase in the total number of users, each potential relay requires a separate orthogonal subchannel in incremental redundancy-based cooperative diversity, hence, making the system bandwidth inefficient. The system utilizing a space-time coded protocol, however, requires all relays to transmit over the same subchannel and is hence 


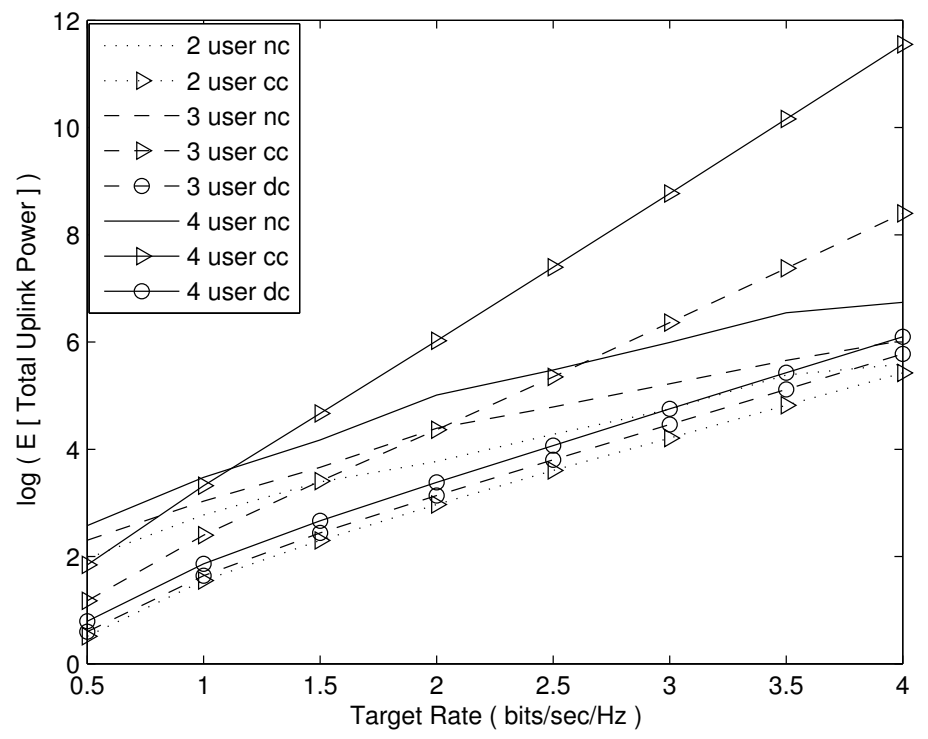

Figure 3.2: Average power consumption in a uplink as a function of target rate for no cooperation (nc), incremental redundancy-based cooperative diversity under code combining (cc), and space-time coded cooperative diversity under diversity combining (dc). The figure indicates that for fewer number of users and target rates of interests, the average total power consumption under both incremental redundancy-based and space-time coded cooperative diversity is significantly less than no cooperation.

bandwidth efficient when compared to incremental redundancy-based protocol. Therefore, the space-time coded protocol offers significant power savings over no cooperation (direct transmission) under the proposed relay selection and power minimization algorithm.

Fig. 3.2 illustrates the average power consumption in a user cooperative uplink and under direct transmission, as a function of target rate on a logarithmic scale. It is observed that incremental redundancy-based cooperative diversity with code combining is better than no cooperation up to target rate of $2 \mathrm{bit} / \mathrm{Hz} / \mathrm{sec}$ for 3 users and up to target rate of $1 \mathrm{bit} / \mathrm{sec} / \mathrm{Hz}$ for 5 users. The figure indicates that for fewer number of users and target rates of interest, the average total power consumption under both incremental redundancy-based and spacetime coded cooperative diversity is significantly less than the direct transmission. For higher target rates and more total users in the uplink, the space-time coded protocol outperforms both direct transmission and incremental redundancy-based cooperative diversity, in terms of power consumption. 


\subsection{Conclusion}

In this chapter, we propose a strategy to minimize the total uplink transmit power in a decode-and-forward (DF) user cooperative uplink such that each user satisfies its target data rate. The proposed iterative algorithm for minimizing the total uplink power jointly performs relay selection for the purposes of cooperation and optimally allocates source and the relay powers. We develop a power minimization scheme for incremental redundancybased cooperative diversity with code combining (of relayed information) and space-time coded protocol with diversity combining. With respect to incremental redundancy-based cooperative diversity, we find that the cooperation is beneficial in terms of minimizing the total uplink power at lower target rates and less number of cooperating users. Significant cooperation gains could be obtained using a space-time coded cooperative diversity protocol over the wide range of target rates and total number of users when using the proposed joint relay selection and power minimization algorithm. A practical approach to network power minimization based on the average channel gains and average rates remains a topic of future work. 


\section{Chapter 4}

\section{Performance of Multiuser}

\section{Cooperation under Non-orthogonal Channels}

In the last chapter, we studied the effect of power allocation on the performance of space-time coded cooperative diversity. The users were assumed to be allocated orthogonal channels for the expositional simplicity. Most prior work on cooperative diversity also assumes the allocation of orthogonal channels to multiple users (inter-user orthogonality) and synchronous communication between the signals transmitted from different cooperating users in the network. For example, the use of cooperative diversity in a cellular uplink was first popularized by Sendonaris et al., [31], where the authors developed a full-duplex, two-user sharing protocol for synchronous code-division-multiple-access (CDMA) using orthogonal spreading codes. However, the assumption of orthogonal spreading codes limits the flexibility of the scheme. Also, choosing orthogonal codes does not achieve orthogonality in asynchronous channels. In [1], the authors developed space-time coded decode-and-forward (DF) protocols and present an information-outage probability analysis of these protocols. The medium-access control protocol suggested in $[1,50]$, allocates orthogonal channels to the transmitting users and also assumes block and symbol synchronization. The assumptions on orthogonal channel allocation and synchronous communication between users may require accurate coordination among the cooperating users, causing significant overhead. The 
authors in [51] design linear multi-user detectors for the synchronous cooperative CDMA uplink using non-orthogonal spreading codes and analyze the performance of various detection strategies under repetition-based full-duplex relaying schemes. The issue of non-orthogonal channel allocation in the context of cooperation has been addressed in $[52,53]$. The authors in [52] apply delay-diversity techniques to single-source cooperative networks which do not require orthogonal channelization or symbol-level timing synchronization. In [53], the authors propose a cooperative transmission technique, where relay nodes act as active scatterers and simply retransmit the source's transmission under very loose synchronization constraints.

From another point of view, the information-theoretic analysis of orthogonal cooperative diversity systems typically assumes Gaussian input symbols, however, practical systems must be constrained to use inputs selected from a finite signal set. The authors in [54] evaluate the impact of modulation constraints on the throughput of point-to-point hybrid-ARQ and suggest the extension to relaying protocols. While information theory has provided insight into the behavior of basic cooperative diversity systems, many issues need to be addressed which include investigating the impact of multiple-access interference (MAI) in multi-user cooperation schemes under non-orthogonal channel allocation and asynchronous communications, assessing the information-outage probability performance of multi-user cooperative diversity under practical modulation constraints, and ascertaining suitable combining techniques at the base station in a multi-user cooperative environment. We address these issues in this chapter. The specific contributions are as follows:

1. We propose a multiuser decode-and-forward (DF) cooperative diversity protocol that operates in an asynchronous CDMA uplink while relaxing the inter-user orthogonality constraint. We address the problem of multi-user relaying where each user first broadcasts its own uniquely spread message and then other users that overhear the broadcast can relay the re-encoded and re-spread message to the base station. The protocol developed here leads to fully distributed cooperation where no inter-user coordination is required and greatly simplifies the medium-access control protocol design.

2. We analyze the information-outage probability performance of the proposed protocol 
in underloaded CDMA, fully-loaded CDMA and overloaded CDMA under diversity combining at the base station in the high-signal-to-noise ratio (SNR) regime.

3. We compare diversity combining (e.g., employing space-time coding) and code combining (e.g., employing incremental redundancy) [55] of the relayed information at the base station using numerical results for the information-outage probability of fully-loaded CDMA uplink. For these combining schemes, no matter how the relayed information is combined with itself, the relayed information is always code combined with the source information. In the former case, the relays simply repeat the source's message and at the base station, these multiple observations are combined using a maximal-ratio diversity combining technique. In the code combining case, the source first transmits a codeword and relays help the source by sending additional redundancy bits. The base station then combines the original codeword and the redundancy bits to decode the source's message.

4. We examine the impact of using practical modulation techniques on the outage probability performance of space-time coded cooperative diversity under fully-loaded CDMA system configuration.

5. Finally, we present a practical system design and provide bit-error-probability simulations under the practical adaptive receiver design.

We compare the outage probability performance of the proposed cooperation scheme under fully-loaded CDMA system configurations and diversity combining with that of Laneman's space-time coded protocol [1] which builds upon inter-user orthogonality and accurate synchronous communication assumptions, using high-SNR approximations. The comparison demonstrates the loss in spectral efficiency of the proposed protocol with respect to Laneman's space-time coded protocol due to non-orthogonal spreading code assignment to each user (which introduces inter-user non-orthogonality), asynchronism between relayed transmissions and the (subpotimal) reception method used in our scheme. However, these assumptions make our system practical and more flexible.

Also, it is well known that code combining is almost always better than the diversity 
combining in non-cooperative networks. This is because when code combining is used, the mutual information of the individual channels is added, while when using diversity combining, signal-to-noise ratios add. Interestingly, the numerical results presented here indicate that in a multi-user cooperative diversity environment, diversity combining of the relayed information from multiple users is nearly as good as code combining because of the associated probabilities of a decoding set, as will be explained in the sequel.

The chapter is organized as follows. Section 4.1 introduces a CDMA cellular uplink model and describes the proposed user cooperation protocol and received signal model under cooperation. Section 4.2 analyzes the performance of the proposed multi-user cooperation protocol in underloaded CDMA, fully-loaded CDMA and overloaded CDMA with diversity combining, while Section 4.3 considers code combining techniques. The outage probability for the modulation constrained case is presented in Section 4.4. We provide numerical results in Section 4.5 and Section 4.7 concludes.

\subsection{System Model}

\subsubsection{Conventional CDMA Uplink}

In direct-sequence code-division multiple-access (CDMA) systems, each user is assigned an individual (orthogonal or non-orthogonal) signature waveform or a spreading code and signals from different users may overlap in both time and frequency. The continuous-time baseband received signal at the base station in a non-cooperative asynchronous CDMA uplink with $K$ active users is given by

$$
r(t)=\sum_{k=1}^{K} \sum_{i=0}^{B-1} x_{k}[i] \alpha_{k} s_{k}\left(t-i T_{s}-\tau_{k}\right)+n(t)
$$

where $B$ is the block length, $T_{s}$ is the symbol period, $n(\cdot)$ is an additive white Gaussian noise process, $x_{k}[i] \in \mathbb{C}$ is the $k$-th user's transmitted symbol with $\mathrm{E}\left\{\left|x_{k}[i]\right|^{2}\right\}=P, \alpha_{k}$ is the flat fading channel coefficient for the channel between $k$-th user and the base station, $s_{k}(t)=\sum_{j=0}^{N-1} c_{k}[j] \psi\left(t-j T_{c}\right)$ is the spreading waveform of $k$-th user where $c_{k}[j] \in\left\{-\frac{1}{\sqrt{N}}, \frac{1}{\sqrt{N}}\right\}$ is the $j$-th element of user $k$ 's spreading code, $\psi(t)$ is a unit-energy transmit pulse shape waveform, $N$ being the processing gain. 


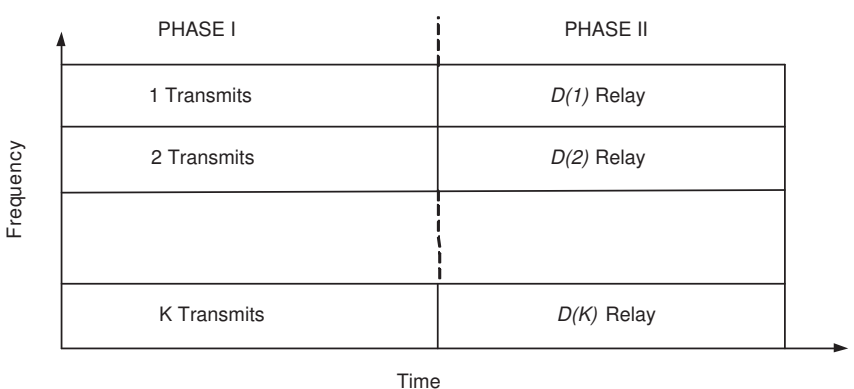

(a)

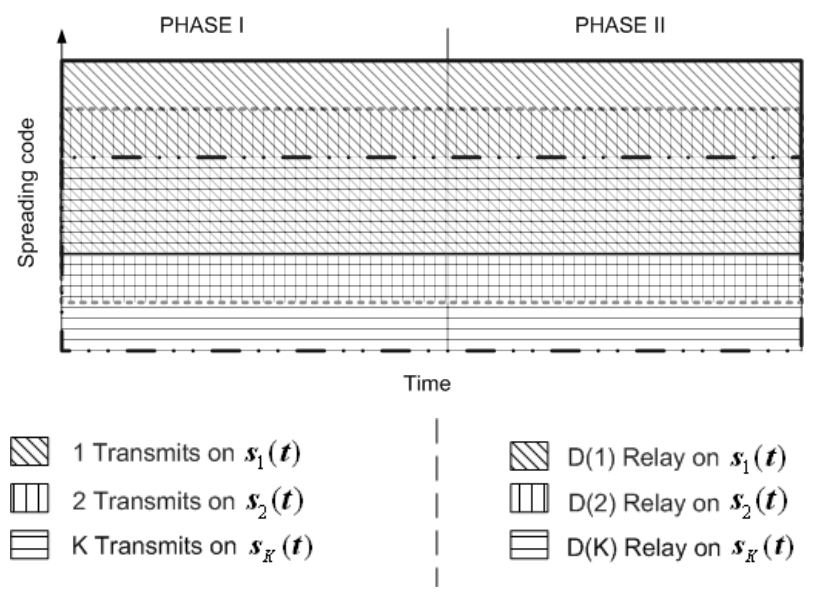

(b)

Figure 4.1: Space-time coded medium-access control for a) Laneman's protocol, and b) the proposed cooperation scheme. Figure indicates example channel allocations across spreading codes and time. For user $k \in\{1,2, \ldots, K\}, \mathcal{D}(k)$ denotes the decoding set. The nonorthogonal spreading waveform of the $k$-th user is denoted by $s_{k}(t)$. Each user now transmits and receives simultaneously on different spreading codes during time Phase I.

CDMA systems may be described as underloaded, fully-loaded, or overloaded. Underloaded CDMA systems arise when the total number of users is less than the processing gain $N$. Fully-loaded CDMA corresponds to the case wherein the number of users is equal to the processing gain. Overloaded CDMA system, in which number of users is larger than the processing gain, is of interest when $N$ cannot be increased due to bandwidth constraints. Overloaded CDMA requires linearly dependent signature waveforms.

\subsubsection{Cooperation in a CDMA Uplink}

\section{Protocol Design}

We analyze a user cooperation protocol wherein users transmit their own data and also serve as relays for other users. This is in contrast with typical relay networks where relays do not have data of their own. We compare the outage probability performance of the proposed cooperation scheme under fully-loaded CDMA and diversity combining of the relayed information with that of Laneman's space-time coded protocol [1], which assumes inter-user orthogonality and accurate synchronous communication. The proposed multi-user cooperation scheme differs from [1] in medium-access control requirements and multiple-access 
strategy. We consider a CDMA cellular uplink consisting of $K$ users. Let set $\mathcal{S}$ denote the set of all users in the system so that $|\mathcal{S}|=K$. Let $m$ be the number of cooperating users and the set of cooperating users be denoted by $\mathcal{C} \subset \mathcal{S}$, where $|\mathcal{C}|=m$. A user is considered to be cooperating if it attempts to decode the transmissions of other users for purposes of forwarding the transmission, regardless of whether the decoding attempt was successful. Let set $\mathcal{N} \triangleq\{\mathcal{S} \backslash \mathcal{C}\}$ denote the set of non-cooperating users. Each source is assigned a particular spreading code. The spreading codes provide processing gain $N$ and are assumed non-orthogonal.

Fig. 4.1(b) depicts channel and subchannel allotments for the proposed CDMA cooperative scheme. The channel representing a single spreading code spans two time-phases and when split into individual time-phases corresponds to subchannels. The transmission between users and the base station is accomplished in two orthogonal time-phases. In the first phase, every user $k \in \mathcal{S}$ broadcasts its message using a particular spreading code (i.e., in the appropriate subchannel). In the second phase, users from the set $\mathcal{C}$ that can decode the $k$-th user's transmission, $k \in \mathcal{C}$, form a decoding set $\mathcal{D}(k)$ and serve as relays $(r)^{1}$. Users in $\mathcal{D}(k)$ are called decoding relays. The decoding relays then transmit to the base station asynchronously, in the appropriate subchannel. The relays in $\mathcal{D}(k)$ could all transmit the same re-encoded and re-spread sequence, which can be diversity combined at the base station. If $N$ is sufficiently large and $\mathcal{D}(k)$ relatively small, then the signals will be transmitted at slightly different times and a RAKE receiver can be used to resolve the individual transmissions. Alternatively, a distributed space-time code could be used as in [1]. As an alternative to retransmitting the same re-encoded sequence, the relays may use incremental redundancy which leads to code combining of the relayed transmissions at the base station. Note that no matter how the relayed information is combined with itself, the relayed information is always code combined with the source information. The users in the set $\mathcal{N}$ continue transmitting their own data in the second phase. For most of the chapter, we will concentrate on the case where $\mathcal{S}=\mathcal{C}$ and hence $m=K$, i.e., every user is a cooperating user, and hence, every user is a potential relay for every other user. The maximum number of decoding relays a particular user can have is $m-1$. Thus for this cooperative diversity

\footnotetext{
${ }^{1}$ We emphasize here that though we use the term 'relay', it also has its own data to transmit.
} 
scheme, decoding relays for any particular source user transmit asynchronously over the same subchannel (i.e., they use the same spreading code). Use of non-orthogonal spreading codes leads to non-orthogonality across the subchannels. Therefore, there exists non-orthogonality across the subchannels and asynchronism within a subchannel. The crux of the problem is then to evaluate performance under these conditions and to design practical coding and reception schemes.

Note: Laneman's protocol [1] assumes half-duplex relays where relays transmit and receive at the same time but this is done on different frequencies. Instead, we assume simultaneous transmission and reception on different spreading codes at the same frequency which may cause transmit signal to overwhelm the receive signal powers leading to self-interference. The self-interference cancelation at the mobile units can be facilitated by the knowledge of relevant antenna gains or use of co-located antennas and/or multiple spreading codes [31].

\section{Received Signal Model}

It is assumed that all the received signals at the base station have the same average power. This kind of power control may not be optimal in reality.

The proposed sharing scheme operates in an asynchronous flat-fading CDMA uplink in the presence of multiple-access interference (MAI) and inter-symbol interference (ISI) due to the use of non-orthogonal spreading codes and asynchronous relayed signals respectively. Consider the signal model for the second phase of transmission. The specified use of decorrelating multiuser detection [11] at the base station effectively transforms the resulting MAI and ISI channel into parallel interference-free scalar flat fading channels with increased background noise. Without loss of generality, we assume that first $m$ users are cooperating users and the remaining $(K-m)$ users are non-cooperating users. The received signal at the base station over a flat fading channel with total $K$ users, $m(<K)$ cooperating users and $m-1$ 


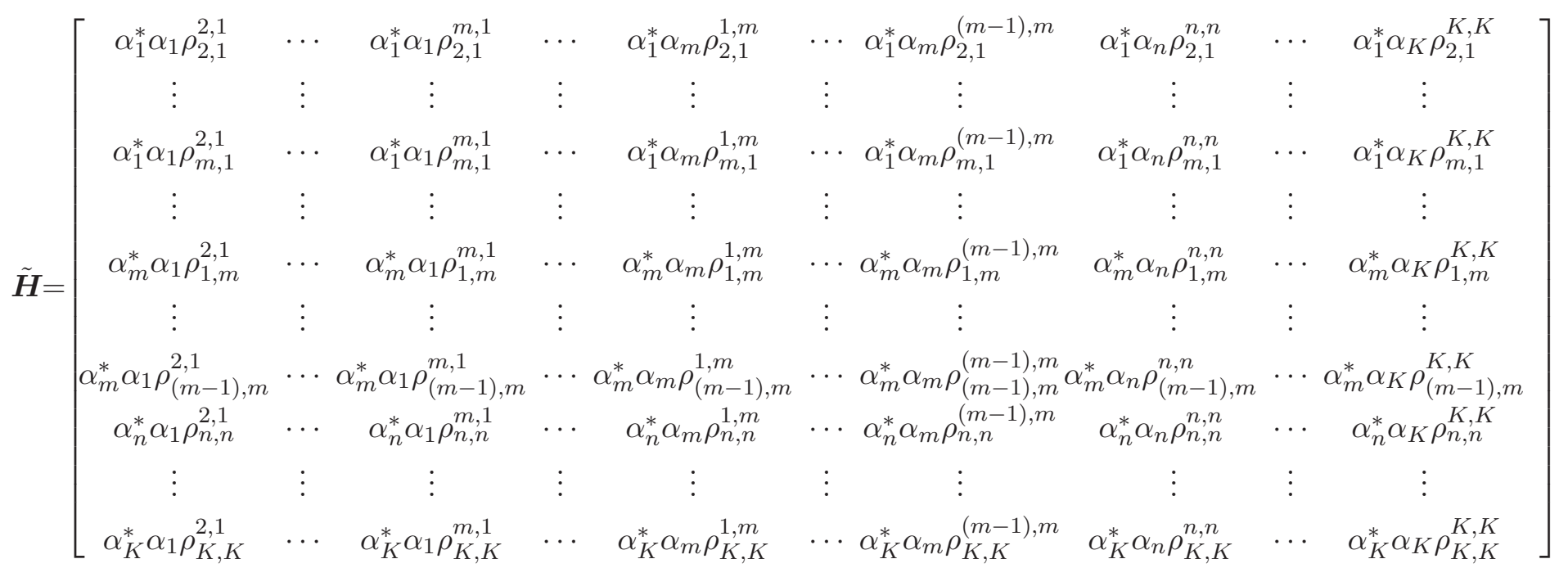

$$
\begin{aligned}
& \boldsymbol{r}=\left[\begin{array}{llllllllll}
r_{2,1} & \cdots & r_{m, 1} & \cdots & r_{1, m} & \cdots & r_{(m-1), m} & r_{n, n} & \cdots & r_{K, K}
\end{array}\right]^{T},
\end{aligned}
$$

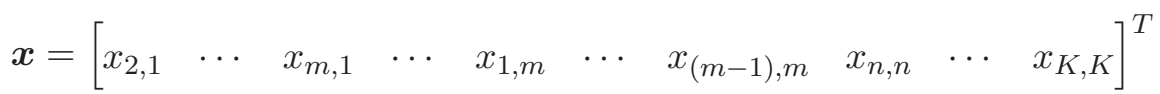

potential relays is given by

$$
\begin{aligned}
r(t) & =\underbrace{\sum_{\substack{k=1 \\
l \neq k \\
l \neq k}}^{m} \sum_{i=0}^{m-1} \sum_{l, k}^{B-1} x_{l, k}[i] \alpha_{l} s_{k}\left(t-i T_{s}-\tau_{l}\right)}_{\text {due to cooperating users }} \\
+ & \underbrace{\sum_{k=m+1}^{K} \sum_{i=0}^{B-1} x_{k, k}[i] \alpha_{k} s_{k}\left(t-i T_{s}-\tau_{k}\right)}_{\text {due to non-cooperating users }}+n(t)
\end{aligned}
$$

where $B, T_{s}, n(\cdot), \tau_{l}$, and $s_{k}(t)$ are as described under equation (4.1). $x_{l, k}[i] \in \mathbb{C}$ is the $k$-th user's coded symbol transmitted from $l$-th cooperating user with $\mathrm{E}\left\{x_{l, k}^{2}[i]\right\}=P, x_{k, k}[i] \in \mathbb{C}$ is the $k$-th non-cooperating users own transmitted data, $\alpha_{l}$ (or $\alpha_{l, d}$ ) is the flat fading Rayleigh channel coefficient for the channel between $l$-th user and the base station with variance $1 / \lambda_{l}$ (or $\left.1 / \lambda_{l, d}\right)$.

At the base station, the received signal is matched-filtered with respect to the delayed 
spreading waveforms as shown in (4.5). By Cameron-Martin formula [8], this process generates sufficient statistics, $r_{l, k}[i]$, given by

$$
\begin{aligned}
r_{l, k}[i] & =\alpha_{l}^{*} \int_{-\infty}^{\infty} r(t) s_{k}\left(t-\tau_{l}-i T_{s}\right) d t \\
& =\sum_{k^{\prime}=1}^{m} \sum_{\substack{l^{\prime}=1 \\
l^{\prime} \neq k^{\prime}}}^{m-1} \sum_{i=0}^{B-1} x_{k^{\prime}, l^{\prime}}[i] \alpha_{l}^{*} \alpha_{l^{\prime}} \rho_{k, l}^{k^{\prime}, l^{\prime}} \\
& +\sum_{k^{\prime}=m+1}^{K} \sum_{i=0}^{B-1} x_{k^{\prime}, k^{\prime}}[i] \alpha_{l}^{*} \alpha_{k^{\prime}} \rho_{k, l}^{k^{\prime}, k^{\prime}}+n_{k, l}[i]
\end{aligned}
$$

where $\rho_{k, l}^{k^{\prime}, l^{\prime}} \triangleq \int_{-\infty}^{\infty} s_{k}\left(t-\tau_{l}-i T_{s}\right) s_{k^{\prime}}\left(t-\tau_{l^{\prime}}-i T_{s}\right) d t$ is the cross-correlation between delayed spreading waveforms. Stacking all matched-filtered outputs and dropping the time index from the model in (4.5), results in

$$
\boldsymbol{r}=\tilde{\boldsymbol{H}} \boldsymbol{x}+\boldsymbol{n}
$$

where $\boldsymbol{n} \sim \mathcal{N}_{c}\left(\mathbf{0}, N_{0} \tilde{\boldsymbol{H}}\right)$. The structure of $\boldsymbol{r}, \tilde{\boldsymbol{H}}$ and $\boldsymbol{x}$ is shown at the top of the page. In (4.2), the index $m+1$ has been indicated by $n$. Equation (4.7) can further be expressed as

$$
r=\underbrace{A R A^{\mathrm{H}}}_{\tilde{H}} x+n
$$

where $\boldsymbol{A}$ is a quasi-block-diagonal matrix and is a function of only channel gains $\alpha_{i}$ 's, and $\boldsymbol{R}$ is a function of cross-correlations between delayed signature waveforms. The expression for $\boldsymbol{R}$ is obtained by extracting only the cross-correlation entries from (4.2) in the form of a matrix. The diagonal entries in quasi-block-diagonal matrix $\boldsymbol{A}$ are the corresponding diagonal channel coefficient elements of (4.2).

While considering $K=m$ case, matrices $\tilde{\boldsymbol{H}}, \boldsymbol{R}, \boldsymbol{A}$ are obtained from the corresponding matrices with last $K-m$ columns and rows removed.

Applying the decorrelating detector to the discrete-time received vector $\boldsymbol{r}$ yields,

$$
\boldsymbol{y}=(\boldsymbol{A R})^{-1} \boldsymbol{r}+\boldsymbol{v}
$$

where $\boldsymbol{v} \sim \mathcal{N}_{c}\left(\mathbf{0}, N_{0} \boldsymbol{R}^{-1}\right)$. Thus a parallel flat fading scalar channel model similar to [1] is obtained as,

$$
y_{i} \triangleq[\boldsymbol{y}]_{i}=\alpha_{i} x_{i}+v_{i}
$$


but with enhanced noise, distributed as $v_{i} \sim \mathcal{N}_{c}\left(0, N_{0}[\boldsymbol{R}]_{i, i}^{-1}\right)$. Using this scalar channel model with an appropriate signal-to-noise ratio parameterization, the proposed scheme can be compared to [1] via outage probability, i.e., the probability that average mutual information (in bits/sec/Hz) falls below a given threshold.

The discrete time received signal at the base station (relays) during the first phase can be written very similar to (4.8) where we now have $\boldsymbol{A}$ as a diagonal matrix (instead of a block diagonal matrix as in phase II) with corresponding source to base station (source to relay) channel gains as diagonal entries. The structure of the correlation matrix $\boldsymbol{R}$ in the first phase is slightly different than in the second phase. Since users transmit their own data only in the first phase, the size of the correlation matrix is $K$ by $K$ while in the second phase it is $m^{2}$ by $m^{2}$ for $K=m$ case and $m^{2}+(K-m)$ by $m^{2}+(K-m)$ for the $K>m$ case, assuming $m-1$ decoding relays.

In general, the complexity of the decorrelating multiuser detector is that of correlationmatrix inversion which is of the order of $\left(m^{2}\right)^{3}$ per user (when $K=m$ ). Also, the assumption that $\boldsymbol{R}$ is invertible is not very restrictive, since a mild assumption that $\boldsymbol{R}$ is positive definite, is sufficient which is the case when the delayed signature waveforms are linearly independent. The situations where $\boldsymbol{R}$ matrix is singular, the decorrelator is not a practicable detector structure [56]. Nevertheless, we use the decorrelator structure here to create performance bounds that would apply to the MMSE multiuser detector case in the high SNR regime, including low-complexity adaptive implementations [57].

Remark: We build in this chapter a framework to exploit the presence of relays which also have their own data to transmit. The benefit of our approach is that it can be used when there is not a rich multipath environment. In effect, the relays create virtual mutlipaths, which can still be exploited by a RAKE receiver. If the actual system has frequency selective fading, which would typically be the case in a CDMA uplink, then even more performance improvements can be expected due to the additional frequency diversity exploited by the RAKE receiver. The work in this chapter applies to frequency selective channels with few modifications in mutual information expressions to be given in the sequel. This can be done by first finding an equivalent SNR for each user to destination channel by summing up the SNRs over that user's multiple resolved paths due to the use of RAKE receiver and 
using this equivalent SNR in mutual information expressions as before. Thus the benefits of cooperation could be obtained by simultaneous exploitation of the channel as well as the potential relays.

\subsection{Performance Under Diversity Combining}

In this section, we study the performance of the proposed cooperative diversity protocol under diversity combining. In this type of cooperation, all the relays in the decoding set for a particular cooperating user transmit on the same subchannel (i.e., on that user's spreading code) using a space-time code or simply delay diversity. The performance measure is information-outage probability, i.e., the probability that the average mutual information $(I)$ between user $k$ and the base station falls below a fixed spectral efficiency $R$. The informationoutage probability serves as a lower bound on the codeword error rate of practically coded systems operating at the same spectral efficiency $R$. Since the decoding set for cooperating user $k, \mathcal{D}(k)$, is a random entity, the outage probability for the channel between user $k$ and the base station is given by

$$
\operatorname{Pr}[I<R]=\sum_{\mathcal{D}(k)} \operatorname{Pr}[\mathcal{D}(k)] \operatorname{Pr}[I<R \mid \mathcal{D}(k)]
$$

We now formulate the outage probability expressions for the space-time coded cooperative diversity in an underloaded CDMA, fully-loaded CDMA and overloaded CDMA uplink under diversity combining at the base station. We indicate different parameters such as fraction of the available degrees of freedom (DOF) utilized by each cooperating terminal, normalized spectral efficiency and normalized discrete-time power in Table 4.1. Because we compare the performance of the proposed scheme to Lanneman's space-time coded protocol, we express the normalized discrete-time power constraint and normalized spectral efficiency for our scheme in terms of the parameters of Laneman's protocol [1]. In Table 4.1, $r$ is the transmission rate in bits/sec, $R$, as defined in [1], is the spectral efficiency in bits/sec/Hz and is nothing but the transmission rate normalized by the fraction of total degrees of freedom utilized by each terminal under Laneman's non-cooperative medium-access protocol. Also, $R_{\mathrm{CDMA}}$ is the normalized spectral efficiency in bits/sec/Hz in case of the proposed scheme 


\begin{tabular}{|l|l|l|l|}
\hline & Laneman[2 & $\begin{array}{l}\text { Underloaded } \\
\text { CDMA }\end{array}$ & $\begin{array}{l}\text { Fully-loaded } \\
\text { and Overloaded } \\
\text { CDMA }\end{array}$ \\
\hline $\begin{array}{l}\text { Fraction of available DOF utilized by } \\
\text { each cooperating terminal }\end{array}$ & $1 / 2$ & $K / 2 N$ & $1 / 2$ \\
\hline $\begin{array}{l}\text { Normalized discrete-time power con- } \\
\text { straint }\end{array}$ & $2 P / K$ & $2 N P / K^{2}$ & $2 P / K$ \\
\hline $\begin{array}{l}\text { Normalized spectral efficiency } \\
\text { (bits/sec/Hz) }\end{array}$ & $R \triangleq K r / W$ & $\begin{array}{l}R_{\text {CDMA }} \triangleq N r / K= \\
N R / K\end{array}$ & $N r / W=N R / K$ \\
\hline
\end{tabular}

Table 4.1: Normalized (by the fraction of available degrees of freedom utilized by each cooperating user) transmit power and normalized (by the fraction of available degrees of freedom utilized by each user under noncooperative transmission) spectral efficiency parameterizations consistent with Laneman [1]. $r$ is the transmission rate in bits/sec and $W$ is the bandwidth in $\mathrm{Hz}$.

and is expressed in terms of $R$ for fair comparison.

\subsubsection{Underloaded CDMA Uplink}

For underloaded system, $K<N$, where $K$ and $N$ are the total number of users and the processing gain respectively. We assume $m=K$, i.e, all the users in the system are cooperating users. Each user in the proposed protocol is assigned a single spreading code for its own data. Since each user sends its own data on its spreading code in the first time phase and also sends other user's data on that user's spreading code in the second phase, each user effectively uses up to $K$ spreading codes while the total number of linearly independent spreading codes available in the system is $N$. Thus each cooperating terminal utilizes $K / 2 N$ of available degrees of freedom in the channel. The $1 / 2$ factor is due to timephase orthogonality where the total time slot is equally divided into Phase I and Phase II. Conditioned on the decoding set $\mathcal{D}(k)$, the mutual information between $k$-th user and base station can be shown to be

$$
\begin{aligned}
I_{\mathrm{u}-\mathrm{CDMA}} & =\frac{K}{2 N} \log \left(1+\frac{2 N S N R}{K^{2}} \frac{\left|\alpha_{k, d}\right|^{2}}{\left[\boldsymbol{R}^{-1}\right]_{k, k}}\right) \\
& +\frac{K}{2 N} \log \left(1+\frac{2 N S N R}{K^{2}} \sum_{r \in \mathcal{D}(k)} \frac{\left|\alpha_{r, d}\right|^{2}}{\left[\boldsymbol{R}^{-1}\right]_{r, r}}\right)
\end{aligned}
$$


where SNR $\triangleq \frac{P}{N_{0}}$ is the signal-to-noise-ratio in the absence of fading. The mutual information in (4.12) is the sum of the mutual informations for two parallel channels, one from the user $k$ to the base station and other from the set of decoding relays, $r \in \mathcal{D}(k)$, to the base station. Note that since we consider the relayed transmissions using a space-time code or delay diversity and diversity combining at the base station, we have a log-sum expression for the second phase. Using a high-SNR approximation developed in [1], the outage probability conditioned on a decoding set and $\boldsymbol{R}$ can be written as ${ }^{2}$

$$
\begin{aligned}
\operatorname{Pr}\left[I_{\mathrm{u}-\mathrm{CDMA}}<R_{\mathrm{CDMA}} \mid \mathcal{D}(k),\right. & \boldsymbol{R}] \sim\left[\frac{2^{\left(\frac{2 N^{2} R}{K^{2}}\right)}-1}{2 N \mathrm{NNR} / K^{2}}\right]^{|\mathcal{D}(k)|+1} \\
& \times \lambda_{k, d}\left[\boldsymbol{R}^{-1}\right]_{k, k} \times \prod_{r \in \mathcal{D}(k)} \lambda_{r, d}\left[\boldsymbol{R}^{-1}\right]_{r, r} \\
& \times A_{|\mathcal{D}(k)|}\left(2^{\left(\frac{2 N^{2} R}{K^{2}}\right)}-1\right)
\end{aligned}
$$

where $A_{n}(t)=\frac{1}{(n-1) !} \int_{0}^{1} \frac{w^{n-1}(1-w)}{1+t w} d w, \quad n>0$.

The mutual information between the $k$-th user and the potential relay $r$ is given by

$$
I_{k, r}=\frac{K}{2 N} \log \left(1+\frac{2 N S N R}{K^{2}} \frac{\left|\alpha_{k, r}\right|^{2}}{\left[\boldsymbol{R}^{-1}\right]_{r, r}}\right)
$$

The potential relay will be able to decode $k$-th user's message if the realized mutual information between user $k$ and the relay $r$ is greater than the fixed spectral efficiency $R_{\mathrm{CDMA}}$.

$$
\begin{aligned}
\operatorname{Pr}[r \in \mathcal{D}(k) \mid \boldsymbol{R}] & =\operatorname{Pr}\left[I_{k, r}>R_{\mathrm{CDMA}}\right] \\
& =\exp \left[-\lambda_{k, r}\left[\boldsymbol{R}^{-1}\right]_{r, r} \frac{2^{\left(\frac{2 N^{2} R}{K^{2}}\right)}-1}{2 N S N R / K^{2}}\right] .
\end{aligned}
$$

The probability of a decoding set is then given by

$$
\begin{aligned}
\operatorname{Pr}[\mathcal{D}(k) \mid \boldsymbol{R}] & =\prod_{r \in \mathcal{D}(k)} \exp \left[-\lambda_{k, r}\left[\boldsymbol{R}^{-1}\right]_{r, r} \frac{2^{\left(\frac{2 N^{2} R}{K^{2}}\right)}-1}{2 N \mathrm{SNR} / K^{2}}\right] \\
& \times \prod_{r \notin \mathcal{D}(k)} 1-\exp \left[-\lambda_{k, r}\left[\boldsymbol{R}^{-1}\right]_{r, r} \frac{2^{\left(\frac{2 N^{2} R}{K^{2}}\right)}-1}{2 N S N R / K^{2}}\right] .
\end{aligned}
$$

\footnotetext{
${ }^{2}$ The proof is similar to [1].
} 
By high-SNR approximation, using Taylor series expansion of (4.17), we get the probability of a decoding set as

$$
\operatorname{Pr}[\mathcal{D}(k) \mid \boldsymbol{R}] \sim\left[\frac{2^{\left(\frac{2 N^{2} R}{K^{2}}\right)}-1}{2 N \mathrm{SNR} / K^{2}}\right]^{K-|\mathcal{D}(k)|-1} \times \prod_{r \notin \mathcal{D}(k)} \lambda_{k, r}\left[\boldsymbol{R}^{-1}\right]_{r, r} .
$$

Combining (4.11), (4.14), and (4.18), the expression for outage probability under highSNR approximation conditioned on $\boldsymbol{R}$, is given by

$$
\begin{aligned}
\operatorname{Pr}\left[I_{\mathrm{u}-\mathrm{CDMA}}<R_{\mathrm{CDMA}} \mid \boldsymbol{R}\right] & \sim\left[\frac{2^{\left(\frac{2 N^{2} R}{K^{2}}\right)}-1}{2 N \mathrm{SNR} / K^{2}}\right]^{K} \times \sum_{\mathcal{D}(k)} \lambda_{k, d}\left[\boldsymbol{R}^{-1}\right]_{k, k} \\
& \times \prod_{r \in \mathcal{D}(k)} \lambda_{r, d}\left[\boldsymbol{R}^{-1}\right]_{r, r} \times \prod_{r \notin \mathcal{D}(k)} \lambda_{k, r}\left[\boldsymbol{R}^{-1}\right]_{r, r} \\
& \times A_{|\mathcal{D}(k)|}\left(2^{\left(\frac{2 N^{2} R}{K^{2}}\right)}-1\right) .
\end{aligned}
$$

Then the final expression for average outage probability is

$$
\operatorname{Pr}\left[I_{\mathrm{u}-\mathrm{CDMA}}<R_{\mathrm{CDMA}}\right]=\mathrm{E}_{R}\left\{\operatorname{Pr}\left[I_{\mathrm{u}-\mathrm{CDMA}}<R_{\mathrm{CDMA}} \mid \boldsymbol{R}\right]\right\}
$$

The expected value in (4.20) is found using Monte-Carlo simulations by averaging (4.19) over realizations of $\boldsymbol{R}$ for a particular choice of a space-time code.

\subsubsection{Fully-loaded CDMA Upink}

Here $m=K=N$. The mutual information and outage probability expressions for the fully-loaded case can be obtained by substituting $K=N$ in (4.12) and (4.19) respectively. For the sake of completeness, we state the expressions for mutual information and outage probability here. The mutual information conditioned on a decoding set is given as

$$
\begin{aligned}
I_{\mathrm{f}-\mathrm{CDMA}} & =\frac{1}{2} \log \left(1+\frac{2 \mathrm{SNR}}{K} \frac{\left|\alpha_{k, d}\right|^{2}}{\left[\boldsymbol{R}^{-1}\right]_{k, k}}\right) \\
& +\frac{1}{2} \log \left(1+\frac{2 \mathrm{SNR}}{K} \sum_{r \in \mathcal{D}(k)} \frac{\left|\alpha_{r, d}\right|^{2}}{\left[\boldsymbol{R}^{-1}\right]_{r, r}}\right) .
\end{aligned}
$$


And the high-SNR approximation for the outage probability yields

$$
\begin{aligned}
\operatorname{Pr}\left[I_{\mathrm{f}-\mathrm{CDMA}}<R_{\mathrm{CDMA}} \mid \boldsymbol{R}\right] & \sim\left[\frac{2^{\left(2^{2 R}\right)-1}}{2 \mathrm{SNR} / K}\right]^{K} \times \sum_{\mathcal{D}(k)} \lambda_{k, d}\left[\boldsymbol{R}^{-1}\right]_{k, k} \\
& \times \prod_{r \in \mathcal{D}(k)} \lambda_{r, d}\left[\boldsymbol{R}^{-1}\right]_{r, r} \times \prod_{r \notin \mathcal{D}(k)} \lambda_{k, r}\left[\boldsymbol{R}^{-1}\right]_{r, r} \\
& \times A_{|\mathcal{D}(k)|}\left(2^{\left(2^{2} R\right)}-1\right) .
\end{aligned}
$$

\subsubsection{Overloaded CDMA Uplink}

For overloaded CDMA system, $K>N$. Notice that we can generate only $N$ linearly independent spreading waveforms. The remaining $K-N$ signatures waveforms are linear combinations of the first $N$ spreading waveforms. Each user thus utilizes up to all available spreading codes. Hence each user utilizes $1 / 2$ of the available degrees of freedom. We consider the following two special subcases.

Case I : $m=K(>N)$

The allowed maximum number of relays in the decoding set in this case is $K-1$. Because of the existence of linearly dependent spreading waveforms in case of overloaded CDMA system, it is not possible to distinguish between individual relay transmissions at the base station. To identify each user and each relay transmission in cooperative overloaded CDMA $(K>N)$, each relay in $\mathcal{D}(k)$ inserts a random delay before transmitting $k$-th user's data to the base station. This allows us to maintain a full-rank signature matrix $\boldsymbol{R}$. The addition of random delays induces the delay diversity effect which is a form of space-time code but is not an optimal space-time code. Though it is not optimal, it is attractive since it is simple in implementation, fully distributed, and scales with increasing numbers of cooperating users [52]. As indicated in Table 4.1, each cooperating terminal utilizes $1 / 2$ of total degrees of freedom in the channel. Conditioned on the decoding set $\mathcal{D}(k)$, the mutual information 
between $k$-th user and base station can be shown to be

$$
\begin{aligned}
I_{\mathrm{o}-\mathrm{CDMA}} & =\frac{1}{2} \log \left(1+\frac{2 \mathrm{SNR}}{K} \frac{\left|\alpha_{k, d}\right|^{2}}{\left[\boldsymbol{R}^{-1}\right]_{k, k}}\right) \\
& +\frac{1}{2} \log \left(1+\frac{2 \mathrm{SNR}}{K} \sum_{r \in \mathcal{D}(k)} \frac{\left|\alpha_{r, d}\right|^{2}}{\left[\boldsymbol{R}^{-1}\right]_{r, r}}\right) .
\end{aligned}
$$

and the realized mutual information between the $k$-th user and the potential relay $r$ is given by

$$
I_{k, r}=\frac{1}{2} \log \left(1+\frac{2 \mathrm{SNR}}{K} \frac{\left|\alpha_{k, r}\right|^{2}}{\left[\boldsymbol{R}^{-1}\right]_{r, r}}\right) .
$$

Following the same procedure as given in the earlier section, the corresponding high-SNR formulation for outage probability conditioned on $\boldsymbol{R}$ is

$$
\begin{aligned}
\operatorname{Pr}\left[I_{\mathrm{o}-\mathrm{CDMA}}<R_{\mathrm{CDMA}} \mid \boldsymbol{R}\right] & \sim\left[\frac{2^{\left(\frac{2 N R}{K}\right)}-1}{2 \mathrm{SNR} / K}\right]^{K} \times \sum_{\mathcal{D}(k)} \lambda_{k, d}\left[\boldsymbol{R}^{-1}\right]_{k, k} \\
& \times \prod_{r \in \mathcal{D}(k)} \lambda_{r, d}\left[\boldsymbol{R}^{-1}\right]_{r, r} \times \prod_{r \notin \mathcal{D}(k)} \lambda_{k, r}\left[\boldsymbol{R}^{-1}\right]_{r, r} \\
& \times A_{|\mathcal{D}(k)|}\left(2^{\left(\frac{2 N R}{K}\right)}-1\right) .
\end{aligned}
$$

and the final expression for average outage probability is

$$
\operatorname{Pr}\left[I_{\mathrm{o}-\mathrm{CDMA}}<R_{\mathrm{CDMA}}\right]=\mathrm{E}_{R}\left\{\operatorname{Pr}\left[I_{\mathrm{o}-\mathrm{CDMA}}<R_{\mathrm{CDMA}} \mid \boldsymbol{R}\right]\right\}
$$

Case II : $m=N(<K)$

Here the number of cooperating users $m$ is less than the total number of users $K$ and is equal to processing gain $N$. Therefore the allowed maximum number of relays in the decoding set is $N-1$. The remaining $(K-N)$ users transmit their own data to the base station independently in both phases, hence, just add interference to the users that cooperate. Again, each user utilizes $1 / 2$ the total degrees of freedom. The expression for outage probability in this case is similar to the case where all users cooperate but only differs in the exponent of the first term in (4.25) which depends upon SNR. The exponent of the first term in equation (4.25) indicates the diversity gain which is equal to $N$ in this case. Since the 
mutual information formula is the same as in case I, we only present the outage probability conditioned on $\boldsymbol{R}$ which is given as

$$
\begin{aligned}
\operatorname{Pr}\left[I_{\text {o-CDMA }}<R_{\mathrm{CDMA}} \mid \boldsymbol{R}\right] & \sim\left[\frac{2^{\left(\frac{2 N R}{K}\right)}-1}{2 \mathrm{SNR} / K}\right]^{N} \times \sum_{\mathcal{D}(k)} \lambda_{k, d}\left[\boldsymbol{R}^{-1}\right]_{k, k} \\
& \times \prod_{r \in \mathcal{D}(k)} \lambda_{r, d}\left[\boldsymbol{R}^{-1}\right]_{r, r} \times \prod_{r \notin \mathcal{D}(k)} \lambda_{k, r}\left[\boldsymbol{R}^{-1}\right]_{r, r} \\
& \times A_{|\mathcal{D}(k)|}\left(2^{\left(\frac{2 N R}{K}\right)}-1\right) .
\end{aligned}
$$

Unconditional outage probability can then be found by taking expectation of (4.26) with respect to $\boldsymbol{R}$.

\subsection{Performance Under Code Combining}

We consider a fully-loaded CDMA system configuration $(m=K=N)$ in this section. The use of decorrelating multiuser detection as discussed in Section 4 allows us to form interference-free scalar flat-fading parallel channels with increased background noise. Thus instead of using the same Gaussian codebook in the form of a space-time code or delay diversity, the relays could employ different Gaussian codebooks to transmit relayed information toward base station. This is equivalent to each relay transmitting a different part of the codeword. This results in a code combining at the base station. Under code combining, the system in the second time phase behaves like a set of $|\mathcal{D}(k)|$ parallel Gaussian channels. The mutual information under code combining and fully-loaded CDMA system configuration conditioned on a decoding set can be given by

$$
\begin{aligned}
I_{\mathrm{f}-\mathrm{CDMA}} & =\frac{1}{2} \log \left(1+\frac{2 \mathrm{SNR}}{K} \frac{\left|\alpha_{k, d}\right|^{2}}{\left[\boldsymbol{R}^{-1}\right]_{k, k}}\right) \\
& +\sum_{r \in \mathcal{D}(k)} \frac{1}{2} \log \left(1+\frac{2 \mathrm{SNR}}{K} \frac{\left|\alpha_{r, d}\right|^{2}}{\left[\boldsymbol{R}^{-1}\right]_{r, r}}\right) .
\end{aligned}
$$

Notice that since we consider relayed transmissions from different Gaussian codebooks and code combining at the base station, we have sum-log expression for the second phase. The mutual information in (4.27) is thus larger than that in (4.21) for same $|D(k)|$ due to Jensen's inequality. Note that the previously published work on cooperative diversity employing code 
combining at the base station requires the existence of parallel channels which is achieved through orthogonal channel allocation. But in our protocol, though the users have been allocated non-orthogonal spreading codes and relayed transmissions occur asynchronously in the same subchannel for each user, the decorrelating multiuser detector allows the creation of virtual parallel channels without a bandwidth penalty though there is a penalty in the signalto-noise-ratio due to entries in the $[\boldsymbol{R}]^{-1}$ matrix. The closed form expression for the outage probability under code combining is not tractable for an arbitrary number of relays. Hence, instead of using high-SNR approximations, we evaluate the outage probability performance of the proposed protocol under code combining via Monte-Carlo simulation and compare it with the simulated performance of the proposed protocol under diversity combining (4.21) in Section 4.5.

\subsection{Performance Under Modulation Constraints}

In the earlier sections, we provided an information-theoretic analysis of multi-user cooperative diversity using Gaussian distributed inputs. The assumption of Gaussian inputs is justifiable if we are dealing with large signalling constellations. But the information-theoretic results need to be extended so as to take into account the effect of practical modulation techniques. In this section, we compute the mutual information under the constraint of uniform input probabilities considering diversity combining at the base station. We consider a fullyloaded CDMA system configuration $(m=K=N)$. To find the expression for mutual information under modulation constraints with the earlier mentioned system parameters, we model the received signal at the base station during two time-phases as follows. In the first phase, user $k$ transmits. The received signal at the base station during first phase after decorrelating multiuser detection can be written as

$$
y_{1}=\frac{\alpha_{k, d}}{\sqrt{[\boldsymbol{R}]_{k, k}^{-1}}} x+n
$$

where $n \sim \mathcal{N}_{c}\left(0, N_{0}\right), x$ is a modulated symbol drawn from the uniform probability distribution and $\mathrm{E}\left\{|x|^{2}\right\}=2 P / K$ as can be seen from Table 4.1. The received signal model pointed out here is very similar to scalar channel model obtained in (4.10) except the scaled factor 
of $1 / \sqrt{[\boldsymbol{R}]_{r, r}^{-1}}$. We note that doing this does not change the received signal-to-noise-ratio (and yields exactly the same mutual information expression given in (4.21)) but enables the separation of SNR from interference terms while plotting the outage probability performance. The mutual information under modulation constraints between $k$-th user and the base station during phase I is [15]

$$
I_{1}=\frac{1}{2}\left(m-\mathrm{E}_{x, y_{1}}\left[\log \frac{\sum_{z \in \chi} p\left(y_{1} \mid z\right)}{p\left(y_{1} \mid x\right)}\right]\right)
$$

where $m=\log _{2} M, M$ is the signal constellation size, $\chi$ denotes the signal set, and $p(y \mid x)$ is the transition probability density function between input $x$ and the output $y$ as defined in [15]. The factor $1 / 2$ outside the $\log$ term is due to the fraction of degrees of freedom utilized by a cooperating terminal in fully-loaded CDMA. Similarly the received signal at the base station during the second phase under modulation constraints due to retransmissions from $K^{\prime}$ relays can be modeled as

$$
\boldsymbol{y}=\left[\begin{array}{c}
\alpha_{2, d} / \sqrt{[\boldsymbol{R}]_{2,2}^{-1}} \\
\vdots \\
\alpha_{r, d} / \sqrt{[\boldsymbol{R}]_{r, r}^{-1}} \\
\vdots \\
\alpha_{K^{\prime}, d} / \sqrt{[\boldsymbol{R}]_{K^{\prime}, K^{\prime}}^{-1}}
\end{array}\right] x+\boldsymbol{n} .
$$

Again, the expression for the mutual information under uniform input probability and diversity combining conditioned on a decoding set is given by

$$
I_{2}=\frac{1}{2}\left(m-\mathrm{E}_{x, \boldsymbol{y}}\left[\log \frac{\sum_{z \in \chi} p(\boldsymbol{y} \mid z)}{p(\boldsymbol{y} \mid x)}\right]\right) .
$$

The overall mutual information conditioned on a decoding set between $k$-th user and the base station is then

$$
I_{m}=I_{1}+I_{2} .
$$

The mutual information between $k$-th user and a potential relay can be formed in a similar fashion which then can be used to find the probability of a decoding set. Using the expression for probability of the a decoding set and the mutual information expression in (4.32), and applying the total probability law in (4.11), we plot the outage probability performance through Monte-Carlo simulation. 


\subsection{Numerical Results}

In all the figures, $N$ denotes the processing gain, and $K$ denotes the total number of users. $m$ is the number of cooperating users. The outage probability curves are plotted for $\lambda_{i, j}=1$. The spreading codes are random and the delays are assumed to be uniformly distributed between 0 and $T_{s}$. The SNR gain or loss of these curves indicates the spectral (bandwidth) efficiency/inefficiency of the protocols and slope of the curves indicates the spatial diversity order. Figs. 4.2, 4.3 and 4.4, indicate the information-outage probability performance of the proposed cooperative diversity protocol under diversity combining with $N=4$ and $R$ $=1 \mathrm{bits} / \mathrm{sec} / \mathrm{Hz}$, using high-SNR approximation, while Figs. 4.5 and 4.6 present the outage probability performance without high-SNR approximation. Fig. 4.2 indicates the outage probability performance in an underloaded and fully-loaded CDMA uplink when $m=K$. It can be seen that the underloaded system is bandwidth inefficient when compared to fullyloaded system. This is because not all available degrees of freedom in the channel are utilized in this system configuration. The outage probability curve for Laneman's space-time coded protocol is also plotted for comparison. Note that Laneman's protocol [1] can only be treated as the fully-loaded scenario $(m=K=N)$ and also assumes inter-user orthogonality, orthogonal space-time coding, block and symbol synchronization and optimal decoding at the base station. Because of these assumptions, there is no interference within a subchannel or across the subchannels for the protocol design built in [1]. The proposed scheme with fully-loaded configuration ( $m=K=N=4$ ) also demonstrates a loss in spectral efficiency with respect to space-time coded protocol developed in [1]. The loss in the performance is because of the use of decorrelating multiuser detection to generate parallel channels at the base station and, consequently, due to inter-user non-orthogonality which arises because of non-orthogonal spreading codes and/or asynchronism addressed in our scheme. The decorrelating multiuser detector can not optimally handle asynchronism and non-orthogonality. The performance penalty due to above mentioned constraints is manifested through $[\boldsymbol{R}]^{-1}$ matrix entries. We make use of non-orthogonal (random) spreading codes because even if we use orthogonal spreading codes, the asynchronism between cooperating users would destroy orthogonality. Also for a given $N$, the number of orthogonal spreading codes is limited to 


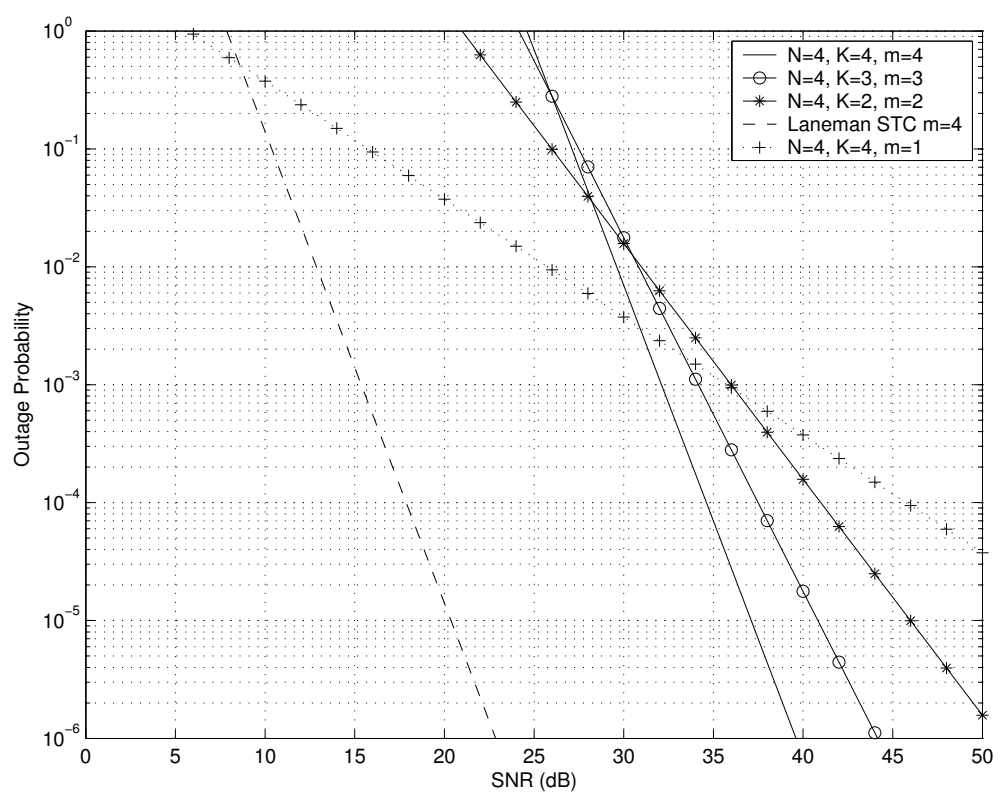

Figure 4.2: Outage probability performance (under high-SNR approximation) of space-time coded scheme in an asynchronous underloaded CDMA uplink with $m=K$ and diversity combining. The threshold spectral efficiency is $R=1 \mathrm{bit} / \mathrm{sec} / \mathrm{Hz}$. The point-to-point CDMA uplink performance $(m=1)$ is shown for comparison. The outage probability curves without high-SNR approximation (dashed lines) are also plotted for comparison.

$N$ unlike non-orthogonal spreading codes. The SNR loss with respect to Laneman could be reduced using alternative receiver structures, e.g., MMSE-DF detection or even optimal multiuser detector, perhaps, as long as post-interference suppression parallel channels can be assumed and modeled. We note here that if we consider cooperation in the proposed fully-loaded CDMA system with orthogonal spreading codes and use of orthogonal spacetime codes under synchronous communication assumption, which yield $\boldsymbol{R}^{-1}=\boldsymbol{I}$ in (4.21) and (4.22), the outage probability performance matches that of Laneman's space-time coded protocol.

Fig. 4.3 compares the outage probability results of the proposed cooperative diversity scheme that operates in the overloaded CDMA $(K>N)$ uplink. The curves are plotted assuming $m=K$ cooperating users. It can be seen that overloading the system is advantageous in terms of the bandwidth efficiency until certain threshold ( $K=6$ in this case). But if the number of users exceed a certain threshold, then it exhibits a loss in bandwidth efficiency. This is because keeping $N$ constant, if we increase the number of cooperating 


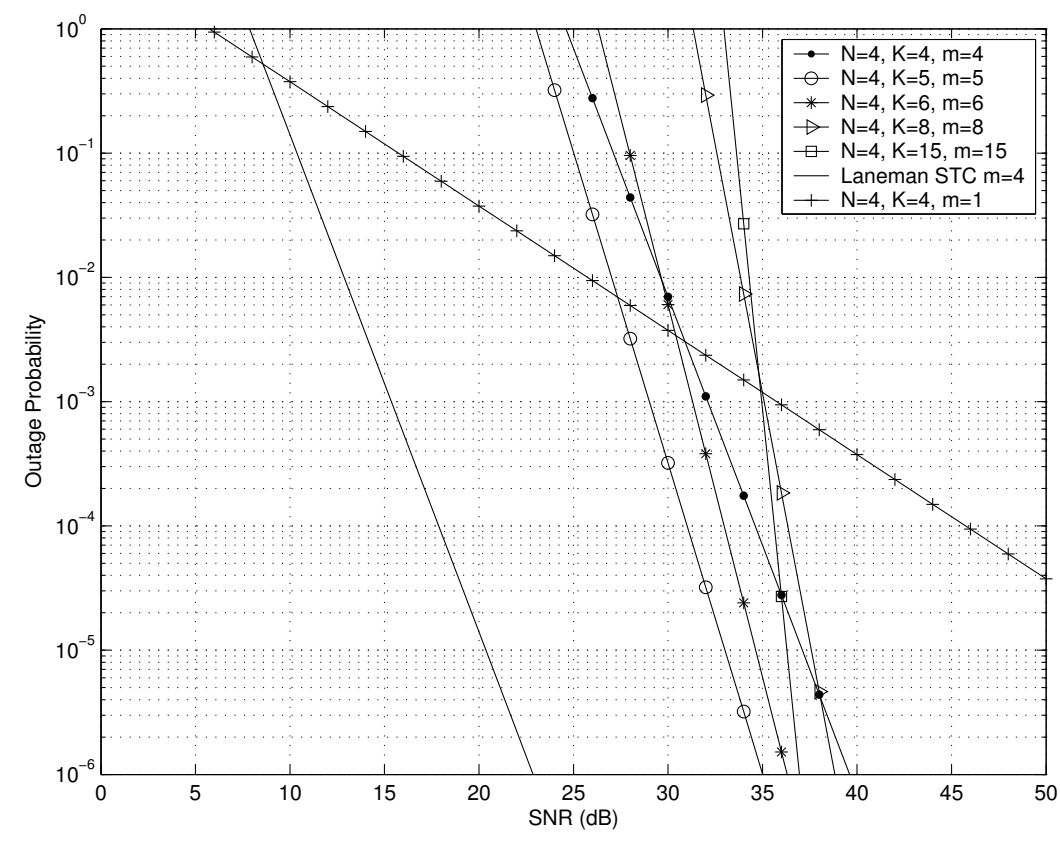

Figure 4.3: Outage probability performance (under high-SNR approximation) of space-time coded scheme in an asynchronous overloaded CDMA uplink $(K>N)$ with $m=K$ and diversity combining. The threshold spectral efficiency is $R=1 \mathrm{bit} / \mathrm{sec} / \mathrm{Hz}$. The point-topoint CDMA uplink performance $(m=1)$ is also shown for comparison.

users $K$ without bound, then $\boldsymbol{R}$ tends towards singularity and leads to large SNR loss. It can also be observed that increasing $K$ increases the diversity order but also increases the SNR loss and hence in practical scenarios, it could be appropriate to choose $K$ slightly larger than $N$.

Fig. 4.4 illustrates the outage probability performance of the proposed space-time coded scheme in overloaded CDMA system assuming $m=N(<K)$ cooperating users. Here, the slope of all outage probability curves is the same because even if we vary total number of users in the system, the number of cooperating users remains fixed which decides the diversity gain and hence the slope of the outage probability. All numerical results via slope of the curves indicate that the protocol achieves full spatial diversity in number of cooperating users.

Fig. 4.5 indicates the conditional outage probability performance comparison between diversity combining and code combining reception schemes for the fully-loaded CDMA cellular uplink with $m=K=N=8$. As we mentioned earlier, we present numerical results instead of high-SNR approximation due to intractability of the closed form expression for 


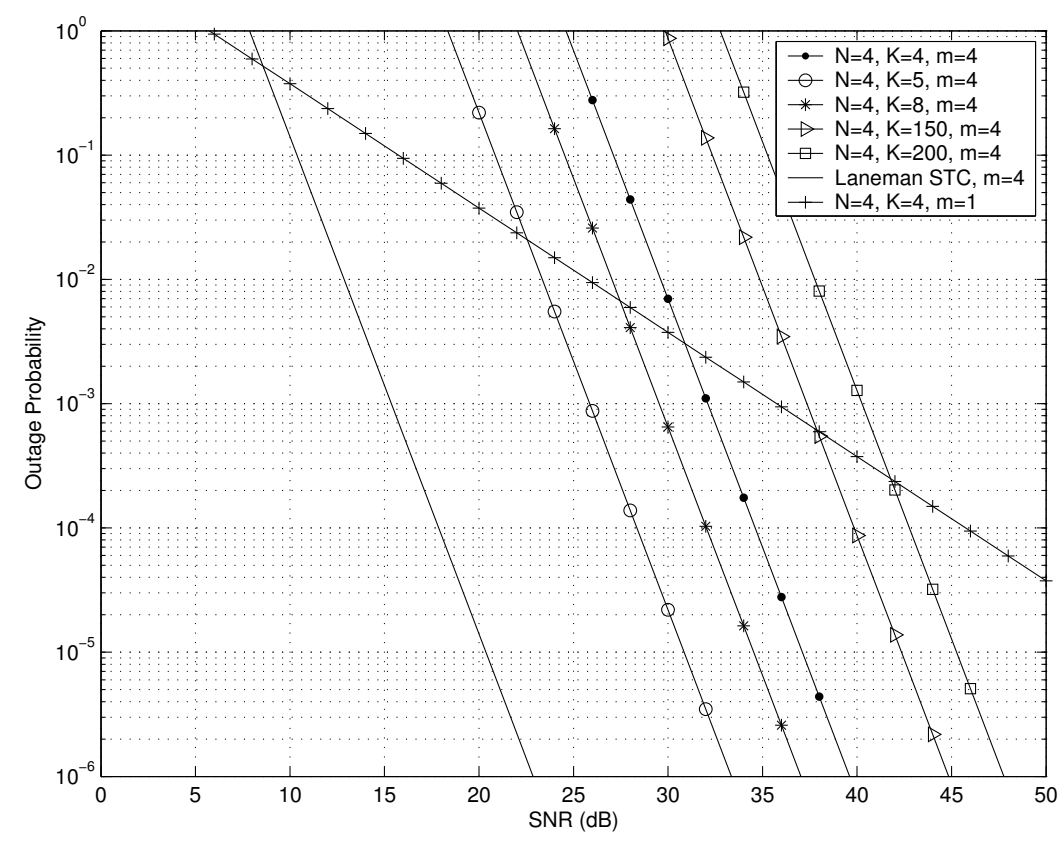

Figure 4.4: Outage probability performance (under high-SNR approximation) of space-time coded scheme in an asynchronous overloaded CDMA uplink $(K>N)$ with $m=N$ and diversity combining. The threshold spectral efficiency is $R=1 \mathrm{bit} / \mathrm{sec} / \mathrm{Hz}$. The point-topoint CDMA uplink performance $(m=1)$ is also shown for comparison.

outage probability in the code combining case. The outage probability is conditioned on $\boldsymbol{R}$ and hence is plotted for one realization of $\boldsymbol{R}$ for simplicity ${ }^{3}$. However, we point out that the relative performance between different outage probability curves is virtually independent of $\boldsymbol{R}$. Recall that $\boldsymbol{R}$ is a function of cross-correlations between delayed spreading waveforms and does not involve channel gains. The results are plotted for $R=1 \mathrm{bit} / \mathrm{sec} / \mathrm{Hz}$. It is well known that code combining is almost always better than the diversity combining in a non-cooperative networks due to the consequence of Jensen's inequality. Interestingly, from the figure, it can be seen that in a cooperative diversity scenario, under the system parameters mentioned here, diversity combining is nearly as good as code combining. Specifically, code combining is only $0.01 \mathrm{~dB}$ better than the diversity combining and this difference is not visible from the figure. This is because decoding set is a random variable. All potential relays in the system do not necessarily decode the source user's transmission. For the SNRs of interest and fewer number of simultaneously active users in the system, the probability of

\footnotetext{
${ }^{3}$ We plot all the conditional outage probability curves using the same realization of $\boldsymbol{R}$.
} 


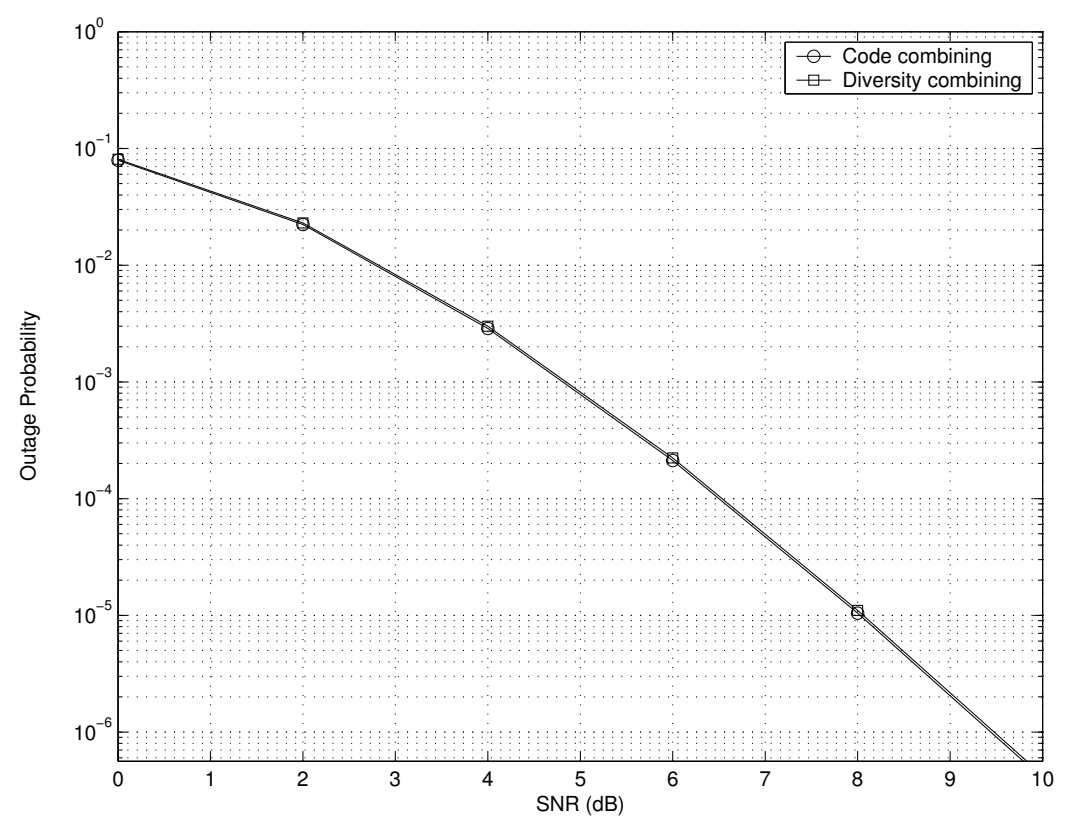

Figure 4.5: Conditional outage probability performance comparison of diversity combining and code combining schemes for fully-loaded CDMA system configuration with $m=K=$ $N=8$. The outage probability is conditioned on $\boldsymbol{R}$. The threshold spectral efficiency is $R$ $=1 \mathrm{bit} / \mathrm{sec} / \mathrm{Hz}$. Code combining is $0.01 \mathrm{~dB}$ better than the diversity combining and so the plots are almost indistinguishable.

having large number of relays in the decoding set is very small and therefore, considering the expansion of (4.11) in the increasing order of $|D(s)|$, only first few terms in the expression (4.11) dominate the system performance. Since the first few terms in diversity combining and the code combining are very similar, the code combining does not offer performance gains (in terms of information-outage probability) over diversity combining. The conclusions might be different if we consider very high-SNR regions and a large pool of users in the system. The conclusions might also change if we consider a non-symmetric network topology where all inter-user channels are statistically different leading to inter-user SNR dependent decoding set probabilities. It was also observed that in a deterministic cooperative network, where $\operatorname{Pr}[D(k)]=1$ for some $D(k)$ (which is the case of a non-cooperative scenario with $|D(k)|$ parallel channels), code combining demonstrates significant performance gain in terms of information outage probability over diversity combining scheme. Thus the probabilities of the decoding sets may drastically affect the outage-probability performance of a cooperation protocol under diversity and code combining schemes. 


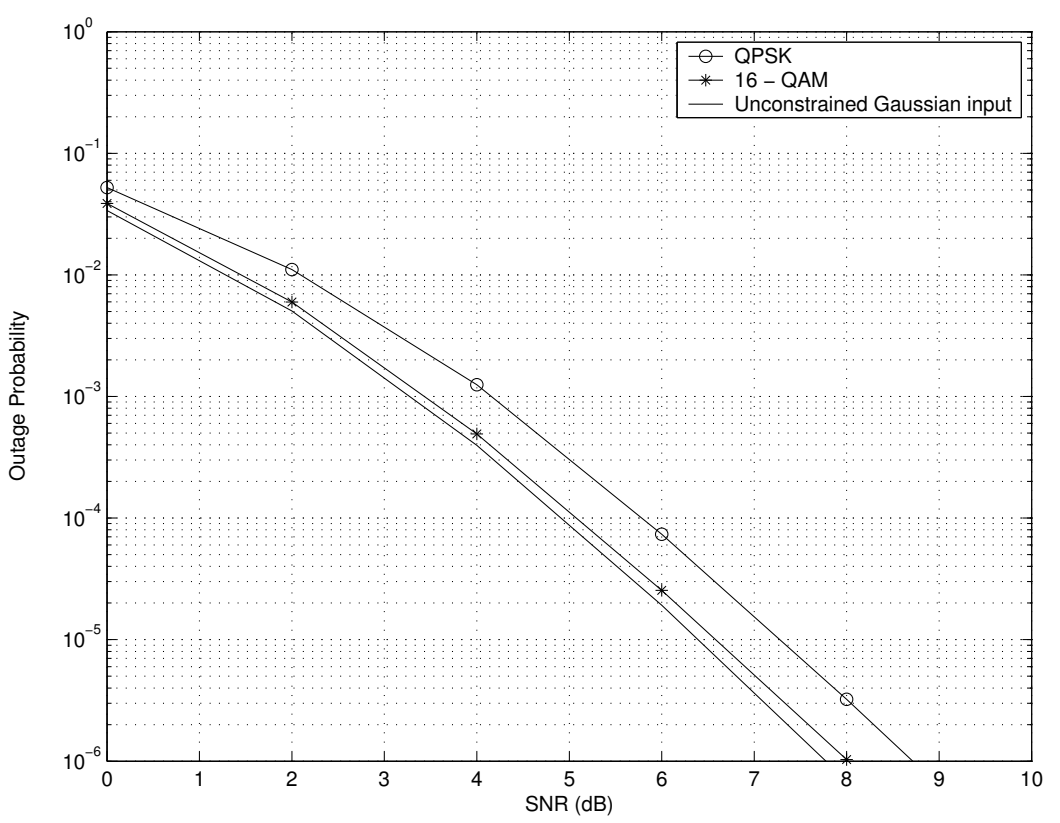

Figure 4.6: Conditional outage probability performance comparison of fully-loaded CDMA system configuration ( $m=K=N=8$ ) under the constraint of uniform input probability (QPSK and 16-QAM modulation) and unconstrained Gaussian input. The outage probability is conditioned on $\boldsymbol{R}$. We assume diversity combining at the base station. The threshold spectral efficiency is $R=0.8 \mathrm{bits} / \mathrm{sec} / \mathrm{Hz}$.

Fig. 4.6 compares the conditional information-outage probability performance of fullyloaded CDMA system (with $m=K=N=8$ ) under modulation constraints and also unconstrained Gaussian input distribution assuming diversity combining at the base station. The information-outage probability is conditioned on $\boldsymbol{R}$ and is plotted without high-SNR approximation. We plot the curves for QPSK modulation and 16-QAM modulation against the threshold spectral efficiency of $0.8 \mathrm{bit} / \mathrm{sec} / \mathrm{Hz}$. It is seen that increasing the signal constellation size renders similar performance to Gaussian input distribution performance at lower rates.

\subsection{Practical System Design}

In the previous sections, we demonstrated the performance of the proposed scheme through an information-theoretic approach. Now we will present a practical approach to system design. Here we consider a specific coding scheme, modulation type and present 
practical pseudo-linear receiver design for the cooperative diversity in an asynchronous cellular uplink.

We present a practical, cooperative DF sharing scheme that works for any number of users in asynchronous environments using orthogonal or non-orthogonal space-time block codes or even using delay diversity technique. The system under consideration makes use of CDMA. We also design a practical adaptive pseudo-linear base-station receiver that jointly separates users while suppressing the multiple-access interference (multiuser detection) and performs space-time decoding (for diversity exploitation) for uplink cooperative communications. The receiver is novel in that, unlike usual MMSE receiver, our base-station receiver incorporates a non-linear complex conjugation receiver front-end in addition to linear MMSE filter and hence is pseudo-linear. The complex conjugation step is critical to obtaining full diversity with linear reception in asynchronism as will be explained in the sequel.

\subsubsection{DF User Cooperation}

We develop a specific protocol for three-or-more cooperating users that makes use of space-time block codes [20]. As an example, we consider a BPSK modulated CDMA system whose users have been assigned to groups of three. Each user sends its own new data in every time slot. Simultaneously, upon successful reception, each user transmits the other user's previous data using a distributed space-time code. For three-user sharing, we use Alamouti's space-time code in our simulations. Note that orthogonal space-time block codes cannot provide full diversity in asynchronous environments for some delay profiles (e.g., if the relative uplink delay between users is exactly one symbol interval), but these profiles occur with probability zero. Because of asynchronism, ML decoding of the orthogonal block codes is no longer linear, but we will see that linear reception, together with a non-linear complex conjugation receiver front-end, still provides full diversity. We also present the bit-error-rate (BER) performance with the delay diversity technique for comparison. 


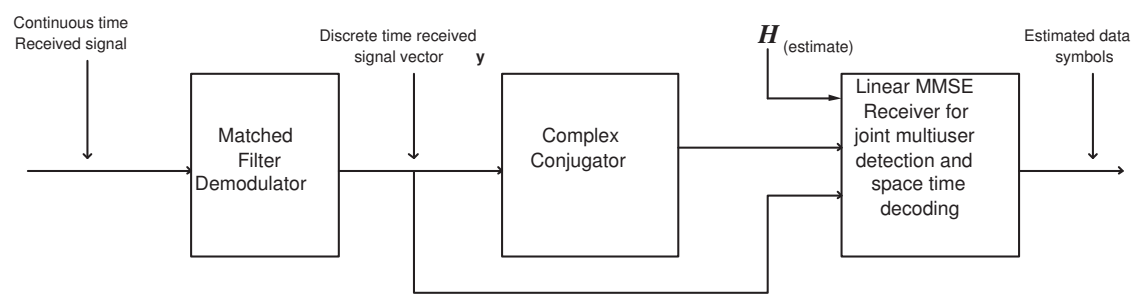

Figure 4.7: Block diagram of the receiver structure

\subsubsection{Adaptive Receiver Design}

We present an adaptive structure based on existing MMSE channel estimation schemes using periodically- inserted pilot symbols. Joint space-time decoding and multiuser detection is accomplished using the receiver structure in Fig. 4.7. The received signal at the basestation with $K$ cooperating users is given by

$$
r(t)=\sum_{k=1}^{K} \sum_{l=1}^{K-1} \sum_{i=0}^{B-1} \beta_{l, k} b_{l, k}[i] \alpha_{l} s_{k}\left(t-i T_{s}-\tau_{l}\right)+n(t)
$$

where $B$ is the frame length, $T_{s}$ is the symbol period, $n(\cdot)$ is the additive white Gaussian noise process, $\beta_{l, k}$ are the Bernoulli random variables having values 0 or 1 and they represent $l$-th user's decision (made via CRC check) on forwarding $k$-th user's space-time coded data, $b_{l, k}[i]$ is $k$-th user's space-time coded symbol transmitted from $l$-th cooperating user, $\alpha_{l}$ and $\tau_{l}$ are the complex Gaussian channel gains with zero mean, unit variance and the delay for the channel between $l$-th user and the destination (base-station) respectively, $s_{k}(t)=$ $\sum_{j=0}^{N-1} c_{k}[j] \psi\left(t-j T_{c}\right)$ is the spreading waveform of $k$-th user where $c_{k}[j]$ is the $j$-th element of user $k$ 's spreading code and $\psi(t)$ is a unit-energy transmit pulse shape waveform.

At the receiver, as shown in Fig.4.7, we first match filter the continuous time received signal with respect to unit energy rectangular chip waveform $\psi(t)$. When we deal with asynchronism (delays $\in\left[0, T_{s}\right]$ ), we match filter the continuous time received signal for one extra time slot. The discrete time received signal vector obtained by stacking all the chipmatched filter outputs can be written as a linear model

$$
\boldsymbol{y}=\boldsymbol{H b}+\boldsymbol{n}
$$

where $\boldsymbol{H}$, a function of the spreading codes and the channel gains, is the effective channel matrix, $\boldsymbol{b}$ is the BPSK symbol vector and $\boldsymbol{n} \sim \mathcal{N}_{c}\left(0, \sigma^{2} \boldsymbol{I}\right)$. We then form a new discrete time 
received signal vector $\tilde{\boldsymbol{y}}$ by stacking complex conjugate version of $\boldsymbol{y}$ with the conventional received signal vector $\boldsymbol{y}$, i.e.,

$$
\begin{aligned}
\tilde{\boldsymbol{y}}=\left[\begin{array}{c}
\boldsymbol{y} \\
\boldsymbol{y}^{*}
\end{array}\right] & =\underbrace{\left[\begin{array}{c}
\boldsymbol{H} \\
\boldsymbol{H}^{*}
\end{array}\right]}_{\tilde{\boldsymbol{H}}} \boldsymbol{b}+\underbrace{\left[\begin{array}{c}
\boldsymbol{n} \\
\boldsymbol{n}^{*}
\end{array}\right]}_{\tilde{\boldsymbol{n}}} . \\
& =\tilde{\boldsymbol{H} b+\tilde{\boldsymbol{n}} .}
\end{aligned}
$$

The complex conjugation step which makes the receiver design novel, is critical to obtaining full diversity with linear reception. Because linear ML detection of orthogonal space-time block codes requires complex conjugates of the received signal, it is reasonable to assume complex conjugates will be helpful for linear detection in asynchronous communication environments. We then perform joint multiuser detection and space time decoding with a linear MMSE filter operating on $\tilde{\boldsymbol{y}}$. Our decision statistic is formed as

$$
\boldsymbol{d}=\left[\left[\tilde{\boldsymbol{H}}_{\text {est }} \tilde{\boldsymbol{H}}_{\text {est }}^{\mathrm{H}}+\sigma^{2} \boldsymbol{I}\right]^{-1} \tilde{\boldsymbol{H}}_{\text {est }}\right]^{\mathrm{H}} \tilde{\boldsymbol{y}}
$$

with bit decisions provided by

$$
\hat{\boldsymbol{s}}=\operatorname{sign}[\operatorname{real}\{\boldsymbol{d}\}] .
$$

$\tilde{\boldsymbol{H}}_{\text {est }}$ is the estimate of the effective channel matrix using least square method.

\subsubsection{Simulation Results}

We use random spreading codes of length 8. The normalized total transmit power of each user during each time slot is 1 . The base-station receiver uses a set of 150 frames of data to estimate the effective channel. The results are included for an estimated channel but we note in passing that an estimated channel case suffers from $1 \mathrm{~dB}$ SNR loss when compared to perfectly known channel case. Fig. 4.8 illustrates the BER and diversity performance of the three-user DF sharing scheme in asynchronous uplink environments for various interuser channel qualities. It is seen that the three-user sharing scheme provides full diversity with $0.1 \%$ inter-user demodulation errors, and provides no diversity with errors $\geq 25 \%$. BER and diversity performance comparison of three-user sharing using Alamouti code and 


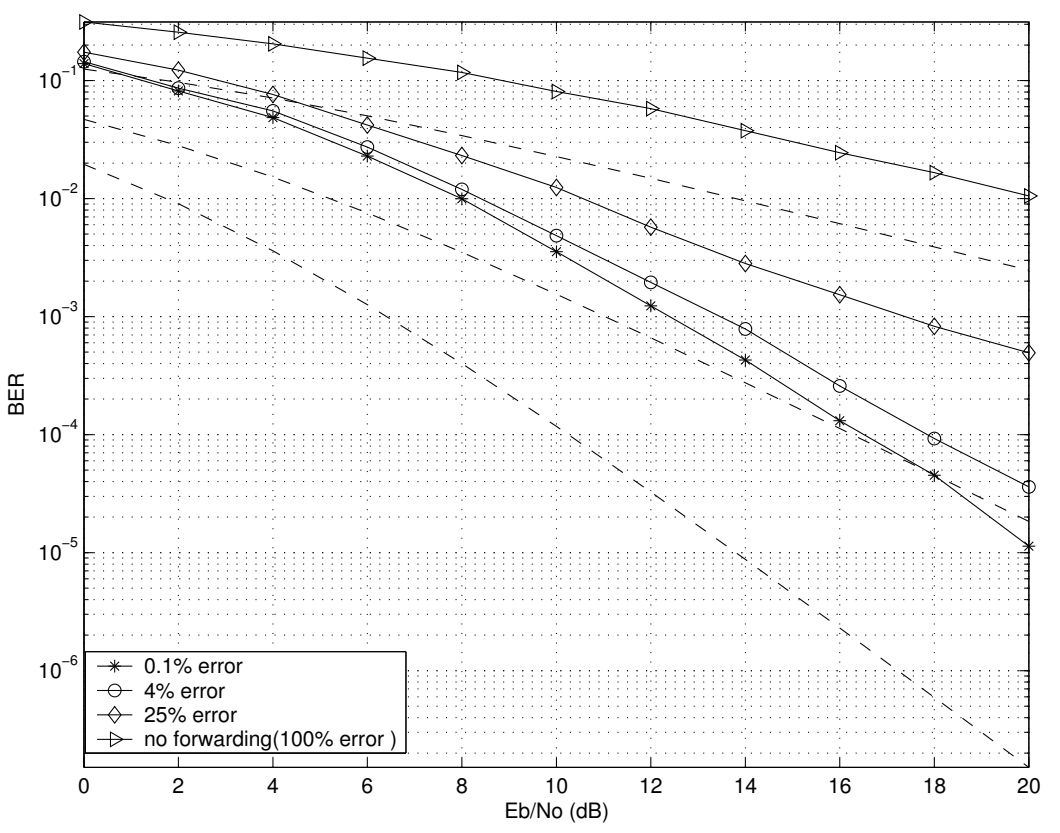

Figure 4.8: BER and diversity performance of three-user DF sharing in an asynchronous uplink for various inter-user channel qualities. Performance of maximum-ratio-combining (MRC) with 1,2 and 3 antennas is plotted for comparison (dotted lines).

delay diversity technique (under perfect inter-user channel assumption) indicates that delay diversity is only $1 \mathrm{~dB}$ worse when compared to Alamouti space-time code and is much simpler to implement, i.e., it's purely distributed.

\subsection{Conclusions}

In this chapter, we have analyzed the performance of cooperative diversity in a CDMA uplink under diversity combining and code combining of the relayed information at the base station while relaxing the inter-user orthogonality and synchronous communication constraints. We have assumed users with full duplex communication capability so that users can transmit and receive simultaneously on the same frequency. Our cooperative scheme employs a sub-optimum decorrelating receiver to suppress the multi-user interference at both the base station and the relay-side. We have evaluated its performance in underloaded, fullyloaded and overloaded CDMA uplink through information-outage probability. The outage probability results under diversity combining indicate that overloaded system is bandwidth efficient up to certain number of users but then exhibits worse performance than fully-loaded 
system as number of users exceed a certain threshold, due to multiple-access interference. We compared diversity combining and code combining of the relayed information at the base station. It is seen that in multi-user cooperation, diversity combining yields almost the same outage probability performance as code combining because not all users in the system act as relays all the time and hence the probabilities of the decoding sets turn out to be a prominent factor in determining the relative performance of code and diversity combining. We also evaluated the performance of multi-user cooperation protocol under the practical modulation techniques. It is observed that increasing the signal constellation size while keeping the target rate constant, we can approach the outage probability performance of a cooperation scheme that uses Gaussian inputs. Looking at all the results, we can argue that the performance loss incurred (with respect to their counterparts) by making the system design much simpler and more practical, for e.g., using diversity combining (instead of code combining), 16-symbol alphabet and a slightly overloaded system, is relatively small.

We also presented a practical system design including design of an adaptive base- station receiver. The simulation results indicated that if the inter-user channel quality is poor, the benefits from cooperation are limited. We demonstrated that delay diversity is nearly as good as orthogonal space-time block codes in asynchronism. 


\section{Chapter 5}

\section{The Performance of Multistage Cooperation}

The previous chapters dealt with dual-stage cooperative diversity schemes where source transmits in the first time phase and then cooperating terminals that can decode source's transmission may transmit a space-time code simultaneously on the same subchannel or use incremental redundancy over orthogonal subchannels during the second phase. These schemes have been shown to extract full diversity in the total number of terminals and not just the number of decoding terminals. However, in many scenarios of two-stage cooperation, not every relay takes part in cooperation because of not being able to decode the source's transmission. Having noted from the last chapter that each relayed transmission leads to either an increase in the average SNR at the destination (as in the case of space-time coded cooperative diversity) or an increase in mutual information at the destination (as in incremental redundancy-based cooperative diversity protocols), it might seem intuitive to design a multi-stage ${ }^{1}$ cooperation scheme where more relays could contribute to the performance enhancement at the destination. Note that the main goal behind developing multi-stage cooperation protocol is to improve the end-to-end mutual information, i.e., achievable rate, not diversity, since full diversity is achieved even with two-stage cooperation schemes.

On the other hand, in a conventional serial multihop transmission without diversity,

\footnotetext{
${ }^{1}$ We use the terms multi-phase and multistage interchangeably, however, they constitute the same network protocol and differ from multihop network protocol.
} 
each relay terminal simply processes the received signal from the immediately preceding terminal and then forwards it to the next along the predefined path. The destination receives a signal only from the last terminal in a single route from source to destination. Thus conventional multihop schemes treat the wireless network as a cascade of point-to-point links with each relay directing its transmission to a single receiver. The main result in [58] showed that in a wireless network with large number of relays, as the number of relays per unit area $n$ increases, the throughput per source-to-destination pair decreases approximately like $1 / \sqrt{n}$. It was implied in [58] that efforts should be targeted at designing networks with small numbers of relays or terminals be connected with nearby relays. The findings of [59] indicated an increase in the throughput of multihop ad-hoc wireless networks when relay nodes are mobile. The outage probability performance of multihop transmission without diversity was studied in [60]. To take advantage of spatial diversity at the relay side, serial multihop diversity relaying was studied in [61], [62]. The authors introduced a concept where each relay exploits received signal not only from the immediate preceding neighbor but from multiple previous relays that repeated the same source message. Each relay in effect receives signals from all preceding transmitting relays, combines them appropriately, processes the combiner output and then re-transmits the source message. Although spatial diversity is exploited in this type of multihop diversity, only a single relay is allowed to transmit in each hop. Another form of mutihop relaying was proposed in [63] wherein source broadcasts its message to a set of potential relays and the relay closest to the destination in turn forwards the message next. This protocol does not take advantage of the transmit diversity in that each potential relay only receives the transmissions from a single previously decoded relay. Once a new relay is selected, all the relays in the network flush their memory of prior transmissions and are, therefore, unable to combine information sent from multiple relays. An improvement to the protocol proposed in [63] was suggested in [64] that exploits transmit diversity effect in a relay network. Similar to [63], the protocol in [64] allows source to broadcast its message to all potential relays in the network. The relay closest to the destination among the set of decoded relays helps source forward its message. Other non-decoding relays keep listening to the transmission from source as well as previous relay transmissions and keep accumulating information and forward the message once they have 
accumulated enough information. Hybrid ARQ was used in conjunction with relaying so that the destination updates on the reception status. The feedback channel was assumed to be error and delay-free. The protocol studied in [64] thus requires location information at the relays and also exhibits overhead in terms of separate feedback channel for the ARQ operation. We take the view that simultaneous relayed transmissions from multiple decoding relays in each hop are beneficial since they increase the received power and transmission range and exploit both transmit as well as receive diversity effect at the relay side. Note that due to the broadcast nature of the wireless medium, more than one relay can receive transmission from the preceding relays that could decode the source message and hence can cooperate simultaneously with other decoding relays using a space-time code.

In the multistage cooperation scheme that we propose, the transmission between source and destination takes place in $T \geq 2$ equal duration and orthogonal time phases. The source transmits only in the first time phase. All relays that can decode the source's transmission forward the source's message to the destination in the second time phase, using a space-time code [20]. Due to the broadcast nature of the wireless medium, other terminals that could not decode the source's transmission earlier listen to the relay transmissions from the previous time phase and keep accumulating information. Relays that have accumulated enough information cooperate next and forward the message to the destination using a space-time code in the next time phase. Other relays keep accumulating the information transmitted by the decoding relays from all previous stages. And the process continues for $T$ cooperation stages. This protocol differs from conventional multihop transmission in that the destination receives independent relayed copies of the transmitted signal from all relays that could decode within a $T$ cooperation phases whereas conventional multihop transmission mandates the destination to receive source's transmission from the last relaying terminal in the path and does not achieve gains due to spatial diversity. The proposed multistage cooperation protocol is thus a generalization of two-stage space-time coded cooperative diversity first analyzed by Laneman et al. in [1] in that, in a proposed multistage cooperation protocol, a new trade-off between an increased number of stages and possible decrease in the mutual information is available for investigation. The primary advantage of multistage relaying is increased mutual information in some network scenarios. When compared to two-stage 
cooperation, possibly more relays can take part in cooperation when multiple stages are employed. The transmission from these additional relays increases the SNR at the destination, increasing the mutual information in effect. However, the penalty for multistage relaying is in the additional resources needed in terms of the radio channels. So the overall effect is immediately not clear. As we will see in the later sections, the use of additional stages may have a negative effect on mutual information which may offset any SNR gain. We are interested in investigating an interesting tradeoff between an increased SNR and decreased spectral efficiency as the number of cooperation stages is increased.

We develop the closed form expression for the outage probability of the proposed multistage cooperation scheme and use it as a performance metric throughout this chapter. The derived outage probability expression is valid for any number of stages and relays. The analytical outage probability expression derived in Section 5.2 provides the optimal number of stages for a given network topology. It can also be used to determine the optimal network topologies. It is seen that the largest multistage cooperation gain in the use of $T \geq 3$ stages over two stages is obtained at low SNR values. The outage probability performance under different relay placement scenarios is also studied. The scenario where the available relays are placed at equal intervals along the straight line between source and destination outperforms most other relay placement strategies for three and four number of stages. For the relay placement scenario where the relays are placed along the straight line between source and destination in an equidistant manner, we also investigate an interesting trade-off between an increased SNR and decreased spectral efficiency as the number of cooperation stages is increased.

\subsection{System Model}

Consider a collection of terminals with source $S$, destination $D$ and $K-1$ relays. For the protocol discussed in this chapter, the transmission between source and destination takes place in $T$ equal duration and orthogonal time phases. We assume half duplex system where users do not transmit and receive simultaneously in the same frequency band. We consider a decode-and-forward cooperation where relays must successfully decode the message before 
forwarding. In the first time phase, the source broadcasts its message which is received by all potential relays. During subsequent time phases, the relays that have successfully decoded the message using information from all previous transmitting relays, transmit a space-time code for the source's message. The use of space-time code allows all relays to transmit the same message in the same band at the same time with no interference. The non-decoding relays keep accumulating SNR and transmit in the later stages when they are able to decode the message. This process repeats for $T$ cooperation stages. Let $I_{r}[m]$ be the mutual information accumulated at relay $r$ in the $m$-th time phase. A relay $r$ is able to decode the message in the $m$-th time phase when $I_{r}[m]>R, R$ being the target rate. Let $\mathcal{D}_{m}(s)$ denote the set of terminals that can decode the source message during the $m$-th time phase so that $\mathcal{D}_{1}(s)=S$. For space-time coded cooperative diversity, the received signal at relay $r$ that has not decoded the message until the $m$-th time phase is

$$
y_{r}[m]=\sum_{k \in\left\{\mathcal{D}_{1}(s), \cdots, \mathcal{D}_{m-1}(s)\right\}} K_{0} \frac{h_{k, r}}{d_{k, r}^{\alpha}} x_{k}+v_{r}[m]
$$

where $h_{k, r}$ is the Rayleigh fading channel coefficient (modeled as zero mean, independent, circularly symmetric Gaussian random variables with unit variances for the channel between terminals $k$ and $r, d_{k, r}$ is the corresponding distance, $K_{0}$ is the signal propagation constant, defined as $K_{0}=\left(\lambda_{c} / 4 \pi d_{0}\right)^{2}$ where $\lambda_{c}$ and $d_{0}$ are the frequency and reference distance and it represents the path loss at an arbitrary reference distance, $\alpha$ is the path loss coefficient and is assumed to be the same for all communication links, $v_{r}[m]$ is the receiver noise distributed as $v_{r}[m] \sim \mathcal{N}_{c}\left(0, N_{0}\right)$ and $x_{k}$ denote the space-time coded symbols transmitted from terminal $k$ with $\mathrm{E}\left\{x_{k}\right\}^{2}=\frac{T}{K} P, P$ being the total transmission power from the source under noncooperative scenario. The corresponding mutual information due to accumulated SNR at relay $r$ in the $m$-th phase is

$$
I_{r}[m]=\frac{1}{T} \log \left(1+\frac{T}{K} \sum_{k \in\left\{\mathcal{D}_{1}(s), \cdots, \mathcal{D}_{m-1}(s)\right\}} g_{k, r} \mathrm{SNR}\right)
$$

where we define SNR $=\frac{P}{N_{0} W}, W$ being the total available bandwidth for the continuous time channel and $g_{k, r}=K_{0} \frac{\left|h_{k, r}\right|^{2}}{d_{k, r}^{\alpha}}$. Note that $g_{k, r}$ are zero mean Gaussian random variables with variances $1 / \lambda_{k, r} \triangleq K_{0} / d_{k, r}^{\alpha}$. The factor $1 / T$ in front of the $\log (\cdot)$ term is because the 
underlined cooperation protocol uses $T$ time phases as against a single time phase used for the transmission in non-cooperative systems. Similarly the factor $T / K$ denotes the fraction of the total power $P$ allowed per relay transmission. The normalization makes comparison between direct transmission and cooperative transmission fair. The multi-stage cooperation protocol can now be described as follows. The source begins its transmission by broadcasting its message during the first time phase. After the source's initial transmission, some of the relays may have decoded the message, particularly those for which $I_{r}[2]>R$. These decoding relays are included in $\mathcal{D}_{2}(s)$. During the next time phase, all relays from $\mathcal{D}_{2}(s)$ transmit a space-time code. The relays that could not decode during the second time phase keep listening to transmissions from relays in $\mathcal{D}_{2}(s)$. Those relays that have $I_{r}[3]>R$ can decode the message and form a decoding set $\mathcal{D}_{3}(s)$. The process continues until $T$ time phases (stages) are reached. The destination keeps information from all relay transmissions received during each time phase.

\subsection{Mutual information and Outage Probability}

In this section, we obtain the closed form expression for the outage probability of multistage cooperation protocol under diversity combining. The performance measure that we use throughout this chapter is information-outage probability, i.e., the probability that the mutual information $I_{S D}$ between source $S$ and the destination $D$ falls below a fixed target rate $R$. Let's first denote the overall decoding set for the transmission between source and destination as $\mathcal{D}(s)$ where $\mathcal{D}(s)=\left\{\mathcal{D}_{1}(s), \cdots, \mathcal{D}_{T}(s)\right\}$. Since the decoding set is a random entity, the outage probability for the channel between source $S$ and the destination $D$ is given by

$$
\operatorname{Pr}\left[I_{S D}<R\right]=\sum_{\mathcal{D}(s)} \operatorname{Pr}\left[I_{S D}<R \mid \mathcal{D}(s)\right] \times \operatorname{Pr}[\mathcal{D}(s)]
$$

Conditioned on decoding set $\mathcal{D}(s)$, the mutual information between source and the destination is

$$
I_{S D}=\frac{1}{T} \log \left(1+\frac{T}{K} \operatorname{SNR} \sum_{r \in \mathcal{D}(s)} g_{r d}\right) .
$$


The end-to-end outage probability for the channel between source and the destination conditioned on decoding set $\mathcal{D}(s)$ is

$$
\begin{aligned}
\operatorname{Pr}\left[I_{S D}<R \mid \mathcal{D}(s)\right] & =\operatorname{Pr}\left[\frac{1}{T} \log \left(1+\frac{T}{K} \sum_{r \in \mathcal{D}(s)} g_{r, d} \mathrm{SNR}\right)<R\right] \\
& =\operatorname{Pr}\left[\sum_{r \in \mathcal{D}(s)} g_{r, d}\right]<\underbrace{\frac{2^{T R}-1}{T S \mathrm{SN} / K}}_{c} \\
& =\sum_{r \in \mathcal{D}(s)} E_{r, d}\left[1-\exp \left(-\lambda_{r, d} c\right)\right]
\end{aligned}
$$

where

$$
E_{r, d}=\prod_{u \in \mathcal{D}(s)} \frac{\lambda_{u, d}}{\lambda_{u, d}-\lambda_{r, d}}, u \neq r
$$

Equation (5.7) is obtained by using the pdf of sum of exponential random variables with distinct means [65].

Probability of a overall decoding set $\mathcal{D}(s)$ is the joint probability that $\mathcal{D}_{2}(s)$ is the decoding set in the second time phase, $\mathcal{D}_{3}(s)$ is the decoding set in the third cooperation phase and so on. Since the composition of $\mathcal{D}_{m}(s)$ depends on $\mathcal{D}_{m-1}, \cdots, \mathcal{D}_{2}$, the above joint probability can be written in the form of chain rule as

$$
\begin{aligned}
\operatorname{Pr}[\mathcal{D}(s)] & =\operatorname{Pr}\left[\mathcal{D}_{1}(s), \cdots, \mathcal{D}_{T}(s)\right] \\
& =\prod_{m=1}^{T} \operatorname{Pr}\left[\mathcal{D}_{m} \mid \mathcal{D}_{m-1}(s), \cdots, \mathcal{D}_{1}(s)\right]
\end{aligned}
$$

As one rule for deciding decoding relays in stage $m$, we require that the realized mutual information at the relay in the $m$-th time phase be greater than the target rate $R$. The realized mutual information at relay $r$ in the $m$-th cooperation phase is

$$
I_{r}[m]=\frac{1}{T} \log \left(1+\frac{T}{K} \sum_{k \in\left\{\mathcal{D}_{1}(s), \cdots, \mathcal{D}_{m-1}(s)\right\}} g_{k, r} \mathrm{SNR}\right) .
$$

Under this rule, we have 


$$
\begin{aligned}
\operatorname{Pr}\left[r \notin \mathcal{D}_{m}(s) \mid r \notin \mathcal{D}_{m-1}(s)\right] & =\operatorname{Pr}\left[I_{r}[m]<R \mid I_{r}[m-1]<R\right] \\
& =\frac{\operatorname{Pr}\left[I_{r}[m]<R\right]}{\operatorname{Pr}\left[I_{r}[m-1]<R\right]} .
\end{aligned}
$$

In the above,

$$
\begin{aligned}
\operatorname{Pr}\left[I_{r}[m]<R\right] & =\operatorname{Pr}\left[\frac{1}{T} \log \left(1+\frac{T}{K} \sum_{k \in\left\{\mathcal{D}_{1}(s), \cdots, \mathcal{D}_{m-1}(s)\right\}} g_{k, r} \mathrm{SNR}\right)<R\right] \\
& =\operatorname{Pr}\left[\sum_{k \in\left\{\mathcal{D}_{1}(s), \cdots, \mathcal{D}_{m-1}(s)\right\}} g_{k, r}\right]<\underbrace{\frac{2^{T R}-1}{T S N R / K}}_{c} \\
& =\sum_{k \in\left\{\mathcal{D}_{1}(s), \cdots, \mathcal{D}_{m-1}(s)\right\}} E_{k, r}\left[1-\exp \left(-\lambda_{k, r} c\right)\right] .
\end{aligned}
$$

Substituting (5.14) in (5.11),

$$
\operatorname{Pr}\left[r \notin \mathcal{D}_{m}(s) \mid r \notin \mathcal{D}_{m-1}(s)\right]=\frac{\sum_{k \in\left\{\mathcal{D}_{1}(s), \cdots, \mathcal{D}_{m-1}(s)\right\}} E_{k, r}\left[1-\exp \left(-\lambda_{k, r} c\right)\right]}{\sum_{k \in\left\{\mathcal{D}_{1}(s), \cdots, \mathcal{D}_{m-2}(s)\right\}} E_{k, r}\left[1-\exp \left(-\lambda_{k, r} c\right)\right]} .
$$

Since each relay makes its decision independently under the above rule and fading coefficients are independent in the system model under consideration, we have

$$
\begin{aligned}
\operatorname{Pr}\left[\mathcal{D}_{m}(s) \mid \mathcal{D}_{m-1}(s), \cdots, \mathcal{D}_{1}(s)\right]= & \prod_{r \notin \mathcal{D}_{m}(s)} \operatorname{Pr}\left[r \notin \mathcal{D}_{m}(s) \mid r \notin \mathcal{D}_{m-1}(s)\right] \\
& \times \prod_{r \in \mathcal{D}_{m}(s)} \operatorname{Pr}\left[r \in \mathcal{D}_{m}(s) \mid r \notin \mathcal{D}_{m-1}(s)\right] \\
= & \prod_{r \notin \mathcal{D}_{m}(s)} \frac{\sum_{k \in\left\{\mathcal{D}_{1}(s), \cdots, \mathcal{D}_{m-1}(s)\right\}} E_{k, r}\left[1-\exp \left(-\lambda_{k, r} c\right)\right]}{\sum_{k \in\left\{\mathcal{D}_{1}(s), \cdots, \mathcal{D}_{m-2}(s)\right\}} E_{k, r}\left[1-\exp \left(-\lambda_{k, r} c\right)\right]} \\
& \times \prod_{r \in \mathcal{D}_{m}(s)}\left\{1-\frac{\sum_{k \in\left\{\mathcal{D}_{1}(s), \cdots, \mathcal{D}_{m-1}(s)\right\}} E_{k, r}\left[1-\exp \left(-\lambda_{k, r} c\right)\right]}{\sum_{k \in\left\{\mathcal{D}_{1}(s), \cdots, \mathcal{D}_{m-2}(s)\right\}} E_{k, r}\left[1-\exp \left(-\lambda_{k, r} c\right)\right]} .\right.
\end{aligned}
$$

We substitute (5.16) in (5.9) to get the expression for the probability of a decoding set $\mathcal{D}(s)$. The equation (5.9) along with (5.7) is then substituted in (5.3) to arrive at the final closed form expression for the end-to-end outage probability as obtained in (5.17). It can be noted that the outage probability not only depends on the target rate, the total number of relays and the number of stages, but also on the particular time stages when individual relays decode and take part in cooperation. 


$$
\begin{aligned}
\operatorname{Pr}\left[I_{S D}<R\right]=\sum_{\mathcal{D}(s)} & \left\{\sum_{r \in \mathcal{D}(s)} E_{r, d}\left[1-\exp \left(-\lambda_{r, d} c\right)\right]\right\} \\
\times & \left\{\prod_{m=1}^{T}\left(\prod_{r \notin \mathcal{D}_{m}(s)} \frac{\sum_{k \in\left\{\mathcal{D}_{1}(s), \cdots, \mathcal{D}_{m-1}(s)\right\}} E_{k, r}\left[1-\exp \left(-\lambda_{k, r} c\right)\right]}{\sum_{k \in\left\{\mathcal{D}_{1}(s), \cdots, \mathcal{D}_{m-2}(s)\right\}} E_{k, r}\left[1-\exp \left(-\lambda_{k, r} c\right)\right]}\right)\right. \\
& \left.\times\left(\prod_{r \in \mathcal{D}_{m}(s)} 1-\frac{\sum_{k \in\left\{\mathcal{D}_{1}(s), \cdots, \mathcal{D}_{m-1}(s)\right\}} E_{k, r}\left[1-\exp \left(-\lambda_{k, r} c\right)\right]}{\sum_{k \in\left\{\mathcal{D}_{1}(s), \cdots, \mathcal{D}_{m-2}(s)\right\}} E_{k, r}\left[1-\exp \left(-\lambda_{k, r} c\right)\right]}\right)\right\} .
\end{aligned}
$$

\subsection{Numerical Results}

In all the figures, $K$ denotes the total number of relays including the source. Path loss coefficient, $\alpha$ is 5 and constant $K_{0}$ is $10^{-4}$ (assuming $\lambda_{c}=2.4 \mathrm{GHz}$ and $d_{0}=1 \mathrm{~m}$ ). Relays are placed in a $1 \times 1$ grid with source located at $(0,0.5)$ and the destination at $(1,0.5)$. Our performance metric is the information-outage probability and is obtained using its expression derived in the previous section. Unless stated, we assume that the target source rate $R$ is 1 $\mathrm{bit} / \mathrm{sec} / \mathrm{Hz}$.

Figures 5.2 and 5.3 present the outage probability performance with $K=8$ under various relay placement scenarios, as described in Fig. 5.1. The number of cooperation stages is varied from 2 to 4 . Based on our numerical tests, it appears that there is no unique relay placement strategy that is best for wide ranges of SNR and for a wide range of $T$ values. However, it can be observed that the relay placement scenario (5) where relays are placed between source and the destination along the straight line in $\mathrm{X}$ axial direction at equal intervals outperforms most of the other candidate relay placement strategies at $T=4$. Under the uniform distribution of relays (scenario 6), $T=3$ gives the best outage probability performance.

Figure 5.4 indicates the outage probability performance when relays are placed along the straight line between source and the destination at equal intervals for different number of relays and at various time stages. Note that it is always advisable to have $K \geq T$ 


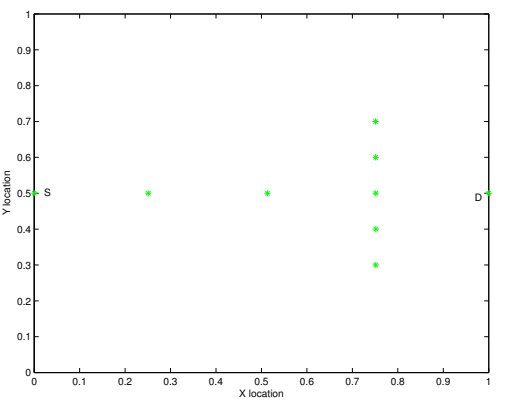

(1)

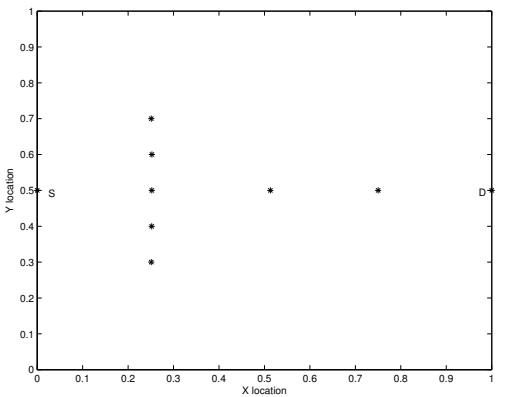

(3)

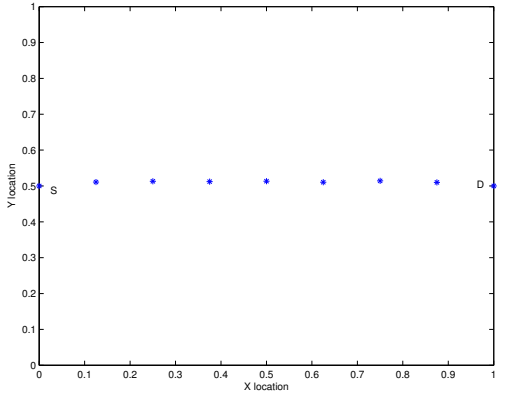

(5)

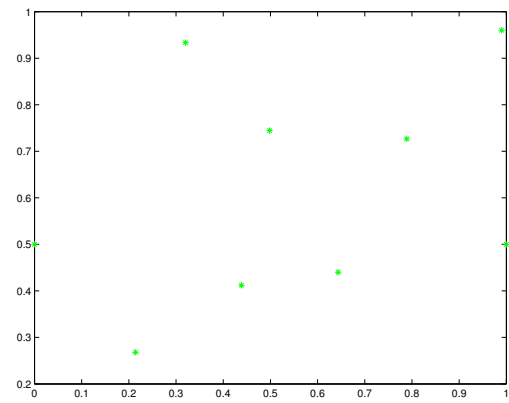

(7)

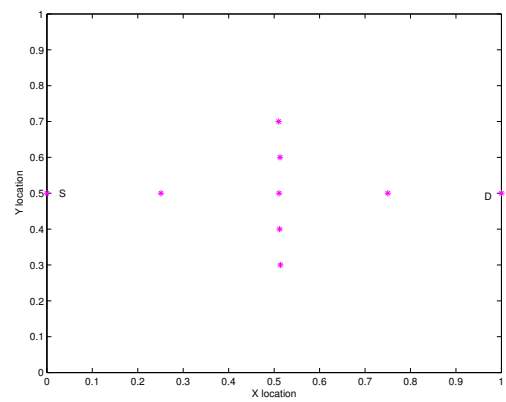

(2)

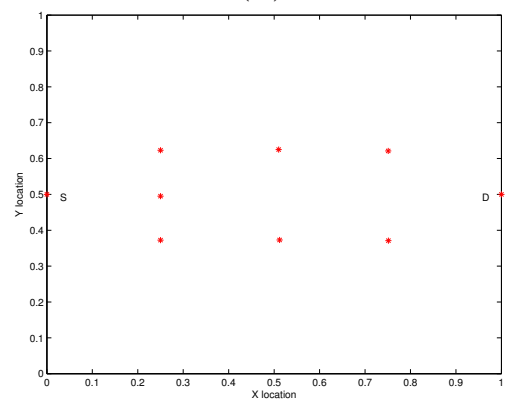

(4)

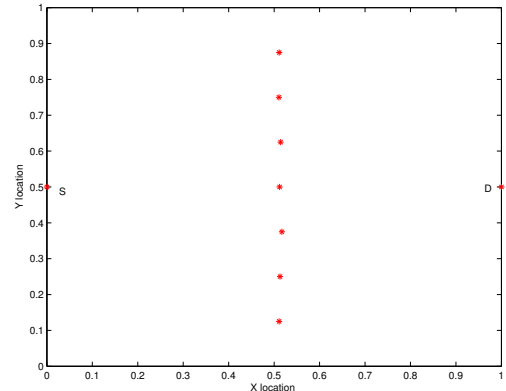

(6)

Figure 5.1: Relay placement scenarios: (1) Array near destination (2) Array in the middle (3) Array near source (4) Equidistant clusters (5) serial equidistant placement between source and destination along $\mathrm{X}$ axis (6) serial equidistant placement between source and destination along $\mathrm{Y}$ axis (7) (sample) Uniform distribution of relays 


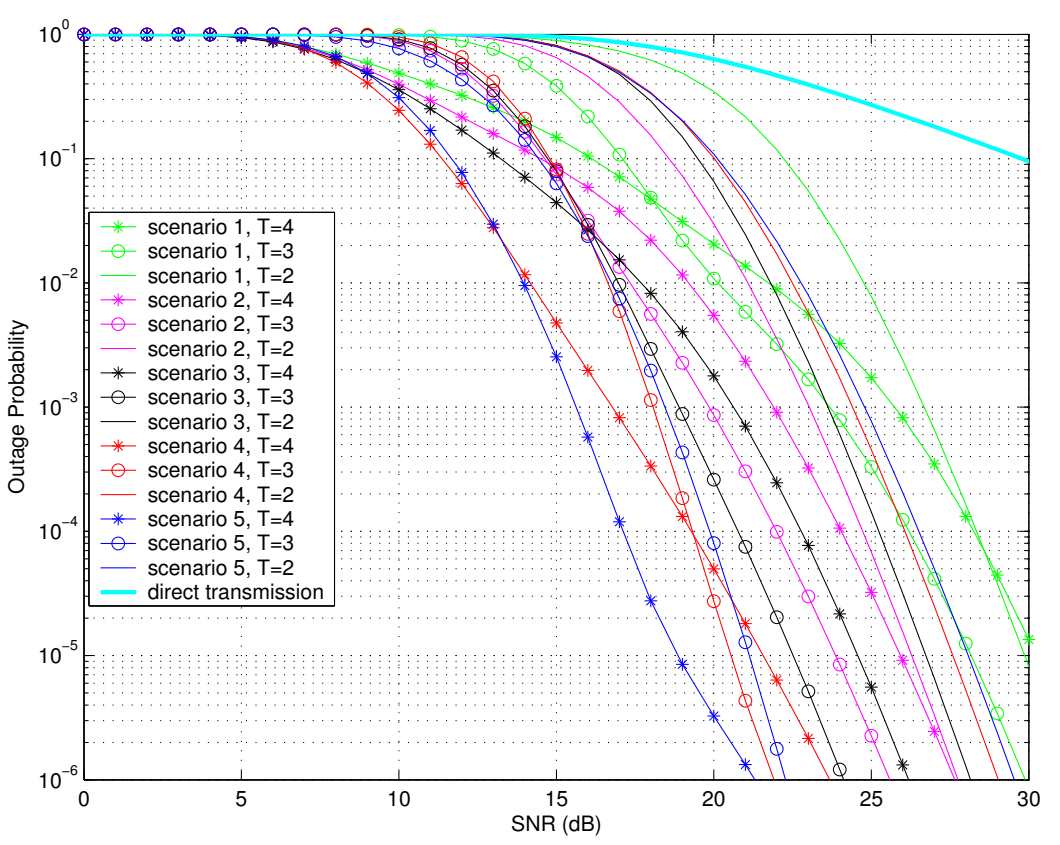

Figure 5.2: Outage probability performance with $K=8$ under various relay placement scenarios and different numbers of cooperation stages.

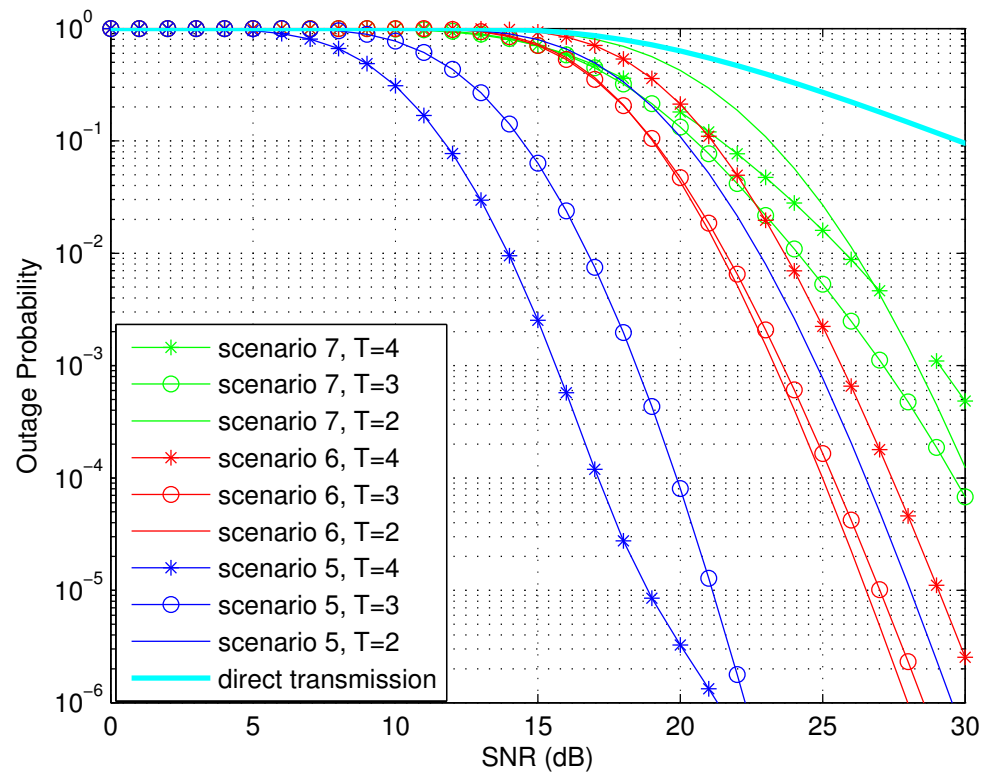

Figure 5.3: Outage probability performance with $K=8$ under various relay placement scenarios and different number of cooperation stages.

because source and every decoding relay transmits only once. With respect to figures 5.2, 5.3, and 5.4, it can be seen that the performance improves with an increase in the number 


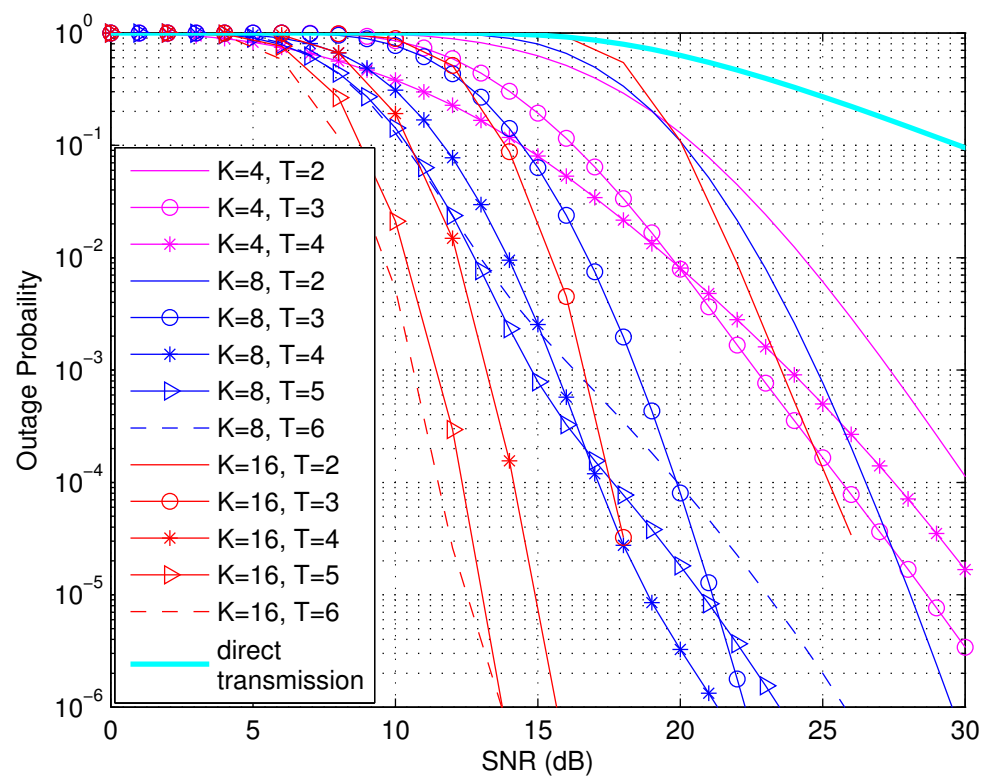

Figure 5.4: Outage probability performance comparison with $K=4, K=8$, and, $K=16$ and $T=2, \cdots, 6$ for Scenario (5).

of cooperation stages until a threshold value for $T$ is reached. The threshold value of $T$ is contingent upon the network topology and the operating SNR values. The improvement in the performance with this increase in the number of stages is obtained at low SNRs, in this case, it corresponds to $10-15 \mathrm{~dB}$ range. This can be explained using the mutual information accumulated at the destination through all $T$ stages. The mutual information at the destination after $T$ stages is $\frac{1}{T} \log \left(1+\frac{T}{K} \sum_{r \in \mathcal{D}(s)} g_{r, d} \mathrm{SNR}\right)$ where $g_{r, d}$ includes the effects of path loss. The mutual information at low SNR increases linearly with SNR with increase in the number of stages. This is because, as number of stages increases, the number of relays in the decoding set also increases, increasing the effective SNR, $\sum_{r \in \mathcal{D}(s)} g_{r, d} \mathrm{SNR}$. At high SNR though, the mutual information increases only logarithmically with SNR and increase in $T$ hurts mutual information because the effect of scale factor $1 / T$ in front of the log term offsets any increase in the SNR. Alternatively, we can say that as we increase $T$, we leave the power-limited regime of the mutual information and enter the bandwidth limited or degree-of-freedom (DOF) limited regime. It is at this point that we obtain the optimal value of $T$.

It can also be seen that the serial placement of relays gives the best performance at 
$T=4$ when compared to other relay location strategies of interest shown in Fig. 5.1. This can be explained as follows. The signal strength, i.e., $\sum_{r \in D(s)} g_{r, d}$ SNR inside the log term in mutual information expression is higher under serial placement compared to other relay placement scenarios presented earlier. This is due to the fact that under serial equidistant placement of relays, increase in $T$ facilitates relays closer to the destination (having small path loss) to be included in the decoding set improving the signal strength significantly and most other relay placement strategies do not exploit this mechanism. Looking at Fig.5.4 it can be observed that having large number of stages with large number of relays does not always help in achieving multistage cooperation gains at low SNR. With respect to serial placement of relays, scenario (5), it is seen that at low SNR, the optimal number of stages for $K=4$ is 3 while for $K=8$ up to $K=16$ case, it is equal to 6 . Table 5.1 indicates the optimal number of stages and the cooperation gain defined here as the ratio of outage probability under no cooperation and outage probability under multistage cooperation at 15 dB SNR.

The outage probability performance for direct transmission (no cooperation) is also shown for comparison. It can be noted that the multistage cooperation schemes always outperform the direct transmission irrespective of the number of relays and the number of cooperation stages because of the induced spatial diversity of the cooperation protocol. Although difficult to indicate in the figures above, theoretically, at infinite SNR, T = 2 case would outperform any $T>2$ outage performance and also the direct transmission outage performance. This is because, at infinite SNR, the probability of all relays being able to decode source's transmission in the second time stage tends to 1 and the addition of cooperation stages does not help improve the performance, but rather suffers from the bandwidth inefficiency problem. Thus at high SNR, the DOF penalty becomes apparent.

Figure 5.5 indicates multistage cooperation gain defined here as the ratio of outage probability under no cooperation and outage probability under multistage cooperation. The multistage cooperation gain is plotted for various path loss coefficient values. Typically, path loss coefficient $\alpha$ varies from 3 up to $6, \alpha=6$ denoting large path loss. It is seen that increasing the number of cooperation stages helps under sever channel conditions, when path loss is higher. 


\begin{tabular}{|l|c|c|c|c|c|c|c|}
\hline Scenario & 1 & 2 & 3 & 4 & 5 & 6 & 7 \\
\hline \hline $\begin{array}{l}\text { Optimal number of cooper- } \\
\text { ation stages }\end{array}$ & 4 & 4 & 4 & 4 & 5 & 3 & 3 \\
\hline $\begin{array}{l}\text { Cooperation gain in dB at } \\
15 \text { dB SNR }\end{array}$ & 8.3 & 10 & 13 & 23 & 31 & 1.51 & 1.31 \\
\hline
\end{tabular}

Table 5.1: Optimal values of $T$ and cooperation gain at $15 \mathrm{~dB}$ SNR for different network topologies shown in Fig. 5.1

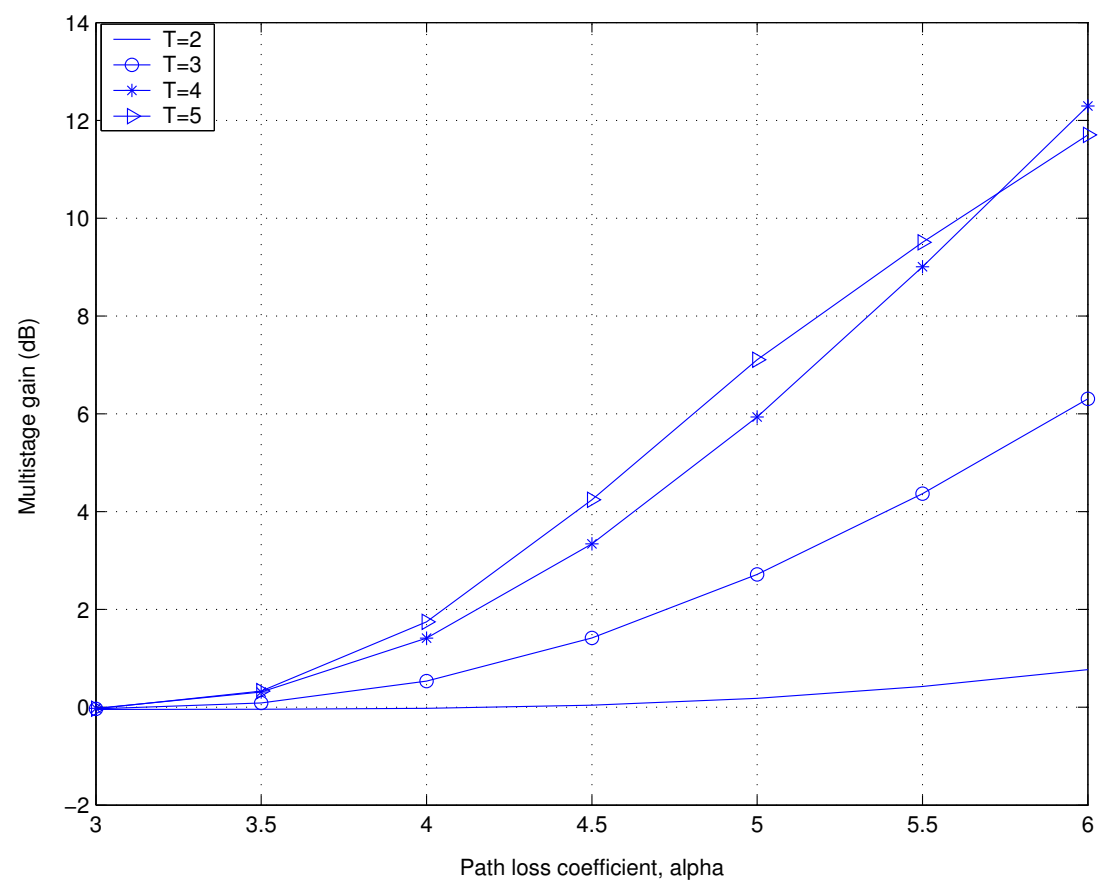

Figure 5.5: Multistage cooperation gain for scenario (5) as a function of path loss coefficient $\alpha$ for $K=8, \mathrm{SNR}=15 \mathrm{~dB}$ and $R=1 \mathrm{bit} / \mathrm{sec} / \mathrm{Hz}$.

The multistage cooperation gain as a function of source target rate is plotted in Figure 5.6. The multistage gain is plotted for larger path loss case. It can be observed that higher multistage gain with respect to direct transmission at low SNR is obtained with five stages at lower rates. At higher rates though, the mutistage gain diminishes even with more stages because of the bandwidth utilization penalty. 


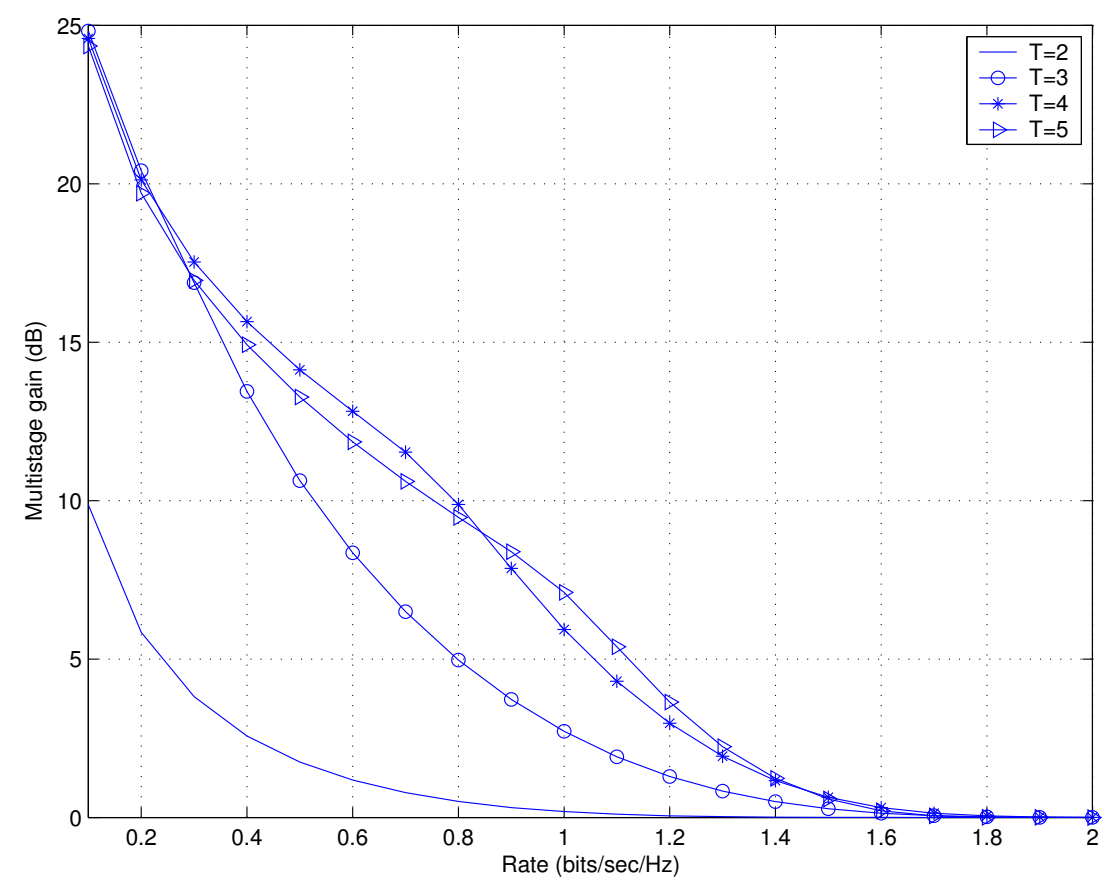

Figure 5.6: Multistage cooperation gain for scenario (5) as a function of target rate $R$ for $K=8$ and $\mathrm{SNR}=15 \mathrm{~dB}$ and $\alpha=5$. Note that the multistage cooperation gain is plotted here for the case of large path loss.

\subsection{Conclusions}

In this chapter, we developed and analyzed the outage probability of multistage cooperation protocol under orthogonal relaying. Through numerical results, we obtained the near-optimal placement of relays that gives the best performance when compared with most other candidate relay location strategies of interest. Under orthogonal relaying and using the outage probability as a performance metric, we showed that near-optimal relay locations are at equal intervals along the straight line between source and the destination. Although in a planar network, this relay placement solution is sub-optimal, for linear topology, it is the optimal solution for relay placement. Having placed the relays along a straight line at equal intervals between source and the destination, it was seen that the largest multistage cooperation gain is obtained at low SNR which increases with increase in the number of stages until certain threshold is reached. The optimal number of cooperation stages $T$ depends on the network topology, total number of relays, the path loss coefficient and the source target rate. The optimal number of cooperation of stages can be found when the mutual information 
curve plotted against SNR leaves the power limited regime and enters the degree-of-freedom limited regime. The optimal number of stages for small number of relays such as $K=4$ case is 3 while for large number of relays, for example, $K=16$ case, it is 6 . It can also be concluded that under very bad channel conditions (higher path loss), multistage cooperation is beneficial when compared with conventional two-stage cooperation schemes. 


\section{Chapter 6}

\section{Summary, Conclusions and Future Work}

In this chapter, we highlight the contributions of our work presented in this dissertation. Cooperation between different terminals in the wireless network has been viewed as a viable option for achieving transmit diversity without the need of implementing multiple antennas at the terminal side. The goal of this research is to evaluate the performance of cooperative communication systems using information theoretic tools under the various scenarios of non-orthogonal channel allocation between cooperating terminals, power allocation and relay selection methods and allocation of multiple stages of cooperation based upon the topology of the network. The objective of this dissertation is to design such cooperative communication schemes and to investigate the energy efficiency-bandwidth efficiency trade-offs.

\subsection{Summary and Conclusions}

Designing energy efficient and bandwidth efficient cooperative communication schemes often leads to design of efficient resource allocation algorithms for cooperative wireless networks. In particular, we look at channel allocation schemes and power allocation schemes in user cooperative wireless systems. The design objective of channel allocation is to maximize the system throughput while at the same time reducing the interference between users. In user cooperative scenarios, it becomes important to address the issue of channel allocation 
since conventional way of allocating a separate channel for each relayed transmission may not be bandwidth efficient. Considering the fact that power is another critical resource, optimizing the usage of this resource is also essential. We consider the problem of allocating optimal power between user's own data and the cooperative data for other users. In the following, we mention the particular areas of study and summarize the results of this work.

Although cooperation between users creates spatial diversity and reduces the power consumption in a wireless network when compared to direct transmission between the source and the destination, equal power allocation between source and the users cooperating with it is not optimal. The set of decoding relays is a function of the source to relay channel conditions as well as the source transmit power. At first, under the scenario of uniform distribution of users and equal power allocation, it looks like having large number of relays that can decode the source's transmission and hence take part in cooperation would lead to energy-efficient transmission of the source message toward the base station. In this case, the possibility of having a relay closer to the base station would be high which would reduce the burden of transmit power at all other cooperating relays. However, in order for the relay closer to the base station (and hence away from the source) to decode the source message, a large transmit power at the source will be required. On the other hand, allowing the source to transmit with less power would yield small number of relays in its decoding set and most probably the ones which are closer to the source. This situation would increase the transmit power burden at all cooperating relays under equal power allocation. Therefore, it is necessary to design a power allocation algorithm that directs the source to transmit with just enough power so that the most efficient set of decoding relays is formed and which also indicates each relay its optimal power to help transmit source's information toward the base station. Assuming users are uniformly distributed in a given area, we developed an algorithm in Chapter 3 that performs relay selection while minimizing the total transmit power and satisfying the data rate of each user in a multiuser, multi-relay decode-and-forward cooperative uplink. We developed a power minimization scheme for incremental redundancy-based cooperative diversity with code combining (of relayed information) and space-time coded protocol with diversity combining. With respect to incremental redundancy-based cooperative diversity, we found that the cooperation is beneficial in terms of minimizing the total uplink power at 
lower target rates and less number of cooperating users. Significant cooperation gains could be obtained using a space-time coded cooperative diversity protocol over the wide range of target rates and total number of users when using the proposed joint relay selection and power minimization algorithm.

As an effort to make the user cooperation schemes more bandwidth efficient and to make the analysis more practical, we investigated the effect of non-orthogonal channel allocation and asynchronous communication on the outage probability performance of two-stage multiuser cooperative diversity in a CDMA uplink. The information-theoretic analysis of orthogonal cooperative diversity systems also typically assumes Gaussian input symbols, however, practical systems must be constrained to use inputs selected from a finite signal set. While information theory has provided insight into the behavior of basic cooperative diversity systems, many issues need to be addressed which include investigating the impact of multipleaccess interference (MAI) in multi-user cooperation schemes under non-orthogonal channel allocation and asynchronous communications, assessing the information-outage probability performance of multi-user cooperative diversity under practical modulation constraints, and ascertaining suitable combining techniques at the base station in a multi-user cooperative environment. We addressed these issues in Chapter 4. Numerical results indicated that the performance loss incurred (with respect to their counterparts) by making the system design much simpler and more practical, for e.g., using diversity combining (instead of code combining), 16-symbol alphabet and a slightly overloaded system, is relatively small.

In Chapter 5, we proposed a multistage cooperation protocol using $T \geq 2$ equal duration and orthogonal time phases. The proposed scheme combines the characteristics of both conventional multihop and two-stage cooperation protocol discussed in the literature. In conventional multihop transmission, each relay upon successful decoding forwards the source message to the next relay in a single route while in the proposed scheme, a set of decoding relays forward the message to the remaining non-decoding relays which in turn can decode and transmit next. It is also an extension of conventional two-stage cooperation scheme where multiple stages for cooperation are allowed so that most of the available relays could eventually decode and contribute to the performance enhancements at the destination. We developed the closed form expression for the outage probability of the proposed 
multistage cooperation scheme and used it as a performance metric. The derived outage probability expression is valid for any number of stages and relays. The analytical outage probability expression derived in Section 5.2 gives insights into the useful relay location strategies. Whenever the relay locations are fixed, it can also be used to find the optimal number of stages through numerical results. It is seen that the multistage cooperation gain is obtained at low SNR values. The outage probability performance under different relay placement scenarios is also studied. The scenario where the available relays are placed at equal intervals along the straight line between source and destination outperforms most other relay placement strategies for three and four number of stages. It is seen that when relays are placed at equal intervals between source and the destination, low SNR cooperation gain increases with increase in the number of stages, however, suffers from a loss after a fixed number of stages is reached, which is a function of total number of relays. We investigated an interesting trade-off of increased SNR and decreased mutual information as the number of cooperation stages is increased.

\subsection{Ideas for Future Work}

The cooperation protocols proposed in the earlier sections along with a power allocation scheme could be generalized further and also could be made more practical by considering the possible extensions of these ideas as discussed in the sequel.

1. Power control algorithm proposed in this chapter can be extended to account for the inter-user non-orthogonality. Under the non-orthogonality constraint, and using the scalar channel obtained after decorrelating multiuser detection as mentioned in Chapter 4, we can arrive at the new mutual information expressions where SNR is scaled by the noise factor and use them as constraints in the proposed power minimization algorithm in (3.5). Under this scenario, more number of relays could help achieve larger mutual information, however, at the same time may hurt the target data rates due to increased multiple-access interference. The evaluation of tradeoff between bandwidth efficient channel allocation and the resulting multiple-access interference and its impact on the power allocation and relay selection would be an interesting topic. 
2. The power minimization algorithm based on instantaneous channel conditions may incur an additional overhead hurting the throughput of the wireless systems under fast fading channel conditions. Practical power control and relay selection methods when only average channel conditions are known to the destination could be developed. In this case, the expectation of the instantaneous mutual information in (3.10) and (3.20) with respect to channel could be taken to set the constraints for average rate for the power minimization algorithm. By Jensen's inequality,

$$
\mathrm{E}\left\{\log \left(1+p_{k} h_{k}^{2}\right)\right\} \leq \log \left(1+\mathrm{E}\left\{h_{k}^{2}\right\} p_{k}\right) .
$$

The above inequality could be used in the constraint equations of the proposed power minimization algorithm for mathematical simplicity. The power control scheme obtained by this method would be practical since the overhead required at the base station in estimating the instantaneous channel gains will be reduced.

3. We proposed a multistage cooperation scheme where the transmission between the source and the destination takes place with the help of relays in $T \geq 2$ equal duration and orthogonal time phases. It would be interesting to observe the effect of assigning unequal time durations for various cooperation stages. Depending upon the network topology and average channel conditions, the duration of each stage can be adapted to yield better outage performance than that of equal duration cooperation stages. The impact of allocating unequal durational cooperation stages on the optimal number of stages for a particular relay placement scenario could be the topic of future study. In fact, the number of stages could be reduced by adjusting the time duration of each stage carefully while still obtaining the multistage gains.

4. It was observed in Chapter 5 that the largest multistage cooperation gain is obtained at low SNR. Since the final expression for the multistage cooperation outage probability is somewhat cumbersome to work with, low SNR approximations for the outage probability could be developed. These may provide further insights on the effect of network topologies on the outage probability performance of multistage cooperation networks. 


\section{References}

[1] N. Laneman and G. Wornell, "Distributed space time coded protocols for exploiting cooperative diversity in wireless networks," IEEE Trans. Inform. Theory, vol. 49, no. 10, pp. 2415-2425, Oct. 2003.

[2] I. Telatar, "Capacity of multi-antenna gaussian channels," European Trans. on Telecommun., vol. 10, no. 6, pp. 585-595, Nov. 1999.

[3] G. Foschini and M. Gans, "On the Limits of wireless communications in a fading environment when using multiple antennas," Wireless Personal Commun., vol. 6, no. 3, pp. 311-335, 1998.

[4] L. Ozarow, S. Shamai, and A. D. Wyner, "Information theoretic considerations for cellular mobile radio," IEEE Trans. Veh. Tech., vol. 43, no. 2, pp. 359 - 378, May 1994.

[5] P. Jung, P. Baier, and Andreas Steil, "Advantages of CDMA and Spread Spectrum Techniques over FDMA and TDMA in Cellular Mobile Radio Applications," IEEE Trans. Veh. Tech., vol. 42, no. 3, pp. 357-364, Aug. 1993.

[6] S. Verdú, Multiuser Detection, Cambridge, UK: Cambridge University Press, 1998.

[7] D. Reynolds, X. Wang, and H.V. Poor, "Blind Adaptive Space-Time Multiuser Detection with Multiple Transmitter and Receiver Antennas," IEEE Trans. Signal Proc., vol. 50, no. 6, pp. 1261-1276, June 2002.

[8] H. V. Poor, An introduction to signal detection and estimation, springer, 1994.

[9] A. Mantravadi and V. V. Veeravalli, "On chi-matched filtering and discrete sufficient statistics for asynchronous band-limited CDMA systems," IEEE Trans. Commun., vol. 49, no. 8, pp. 1457-1467, Aug. 2001.

[10] X. Wang and H. V. Poor, Wireless Communication Systems: Advanced Techniques for Signal Reception, Prentice Hall , 2003.

[11] S. Moshavi, "Multi-user detection for DS-CDMA communication," IEEE Commun. Magazine, vol. 34, no. 10, pp. 124-136, Oct. 1996.

[12] C. Shannon, "A mathemcatical theory of communication," Bell Labs Technical Journal, vol. 27, pp. 379423, Oct. 1948. 
[13] T. M. Cover, and J. A. Thomas, Elements of Information Theory, Wiley-Interscience, 1991.

[14] D. Tse and P. Viswanath, Fundamentals of Wireless Communication, Cambridge University Press, 2005.

[15] G. Caire, G. Taricco, and E. Biglieri, "Bit-interleaved coded modulation," IEEE Trans. Inform. Theory, vol. 44, no. 3, pp. 927-946, May 1998.

[16] A. Paulraj, R. Nabar, D. Gore, Introduction to space-time wireless communications, Cambridge University Press, 2003.

[17] E. Biglieri, R. Calderbank, A. Constantinides, A. Goldsmith, A. Paulraj, H. V. Poor, MIMO Wireless Communications, Cambeidge University Press, 2007.

[18] A. Paulraj, D. Gore, R. Nabar, and H. Bölcskei, "An overview of MIMO communications - A key to gigabit wireless," Proc. IEEE, vol. 92, no. 2, pp. 198-218, Feb. 2004.

[19] S. Alamouti, "A simple transmit diversity technique for wireless communications," IEEE J. Select. Areas Commun., vol. 16, no. 8, pp. 1451-1458, Oct. 1998.

[20] V. Tarokh, H. Jafarkhami, and A. R. Calderbank, "Space-time block codes from orthogonal designs," IEEE Trans. Inform. Theory, vol. 45, no. 5, pp. 1456-1467, July 1999.

[21] V. Tarokh, H. Jafarkhami, and A. R. Calderbank, "Space-time block coding for wireless communications: Performance results," IEEE Trans. Inform. Theory, vol. 17, no. 3, pp. 451-460, Mar. 1999.

[22] V. Tarokh, N. Seshadri, and A. R. Calderbank, "Space-time codes for high data rate wireless communications: Performance criterion and code construction." IEEE Trans. Inform. Theory, vol. 44, no. 2, pp. 744-765, Mar. 1998.

[23] L. Zheng and D. N. C. Tse, "Diversity and multiplexing: A fundamental tradeoff in multiple-antenna channels," IEEE Trans. Inform. Theory, vol. 49, no. 5, pp. 1073-1096, May 2003.

[24] T. Cover and A. El Gamal, "Capacity theorems for the relay channel," IEEE Trans. Inform. Theory, vol. 25, no. 5, pp. 572-584, Sept. 1979.

[25] A. Nosratinia, T.E. Hunter, and A. Hedayat, "Cooperative communication in wireless networks," IEEE Commun. Magazine, vol. 42, no. 10, pp. 74-80, Oct. 2004.

[26] A. Høst-Madsen, "On the capacity of wireless relaying," in Proc. IEEE Veh. Tech. Conf. (VTC), Vancouver, BC, Sept. 2002, vol. 3, pp. 1333-1337.

[27] B. Schein and R. Gallager, "The Gaussian parallel relay network," in Proc. IEEE Int. Symp. on Inform. Theory (ISIT), Italy, June 2000. 
[28] M. Gastpar and M. Vetterli, "On the capacity of large Gaussian relay networks," IEEE Trans. Inform. Theory, vol. 51, no. 3, pp. 765-779, March 2005.

[29] M. Gastpar and M. Vetterli, "On the capacity of wireless networks: The relay case," in Proc. INFOCOM, 2002, vol. 3, pp. 1577-1586.

[30] M. Khojastepour, A. Sabharwal, and B. Aazhang, "On the capacity of 'cheap' relay networks," in Conf. on Inform. Sciences and Sys. (CISS), Baltimore, MD, Apr. 2003.

[31] A. Sendonaris, E. Erkip, B. Aazang, "User cooperation diversity - Part I: System description," IEEE Trans. Commun., vol. 51, no. 11, pp. 1927-1938, Nov. 2003.

[32] A. Sendonaris, E. Erkip, B. Aazang, "User cooperation diversity - Part II: Implementation aspects and performance analysis," IEEE Trans. Commun., vol. 51, no. 11, pp. 1939-1948, Nov. 2003.

[33] T. Hunter, S. Sanayei, and A. Nosratinia, "Outage analysis of coded cooperation," IEEE Trans. Inform. Theory, vol. 52, no. 2, pp. 375-391, Feb. 2006.

[34] B. Zhao and M.C. Valenti, "Distributed turbo coded diversity for the relay channel," IEE Electronics Letters, vol. 39, no. 10, pp. 786-787, May 2003.

[35] A. Nosratinia and T. Hunter, "Grouping and partner selection in cooperative wireless networks," IEEE J. Select. Areas Commun., vol. 25, no. 2, Feb. 2007.

[36] Z. Lin, E. Erkip, and A. Stefanov, "Cooperative regions and partner choice in coded cooperative systems," IEEE Trans. Commun., vol. 54, no. 7, July 2006.

[37] A. Bletsas, A. Khisti, D.P. Reed, A. Lippman, "A Simple Cooperative Diversity Method based on Network Path Selection," IEEE J. Select. Areas Commun., vol. 24, no. 3, pp. 659-672, March 2006.

[38] E. Beres and R. S. Adve, "Selection cooperation in multi-source networks," IEEE Trans. Wireless Comm., vol. 7, no. 1, pp. 118-127, Jan. 2008.

[39] Y. Liang and V.V. Veeravalli, "Resource allocation for wireless relay channels," in Proc. Asilomar Conf. Signals, Systems, Computers, Nov. 2004, vol. 3.

[40] E. Larsson and Y. Cao, "Collaborative transmit diversity with adaptive radio resource and power allocation," IEEE Commun. Letters, vol. 9, no. 6, pp. 511-513, June 2005.

[41] N. Ahmed, M. Khojastepour, and Behnaam Aazhang, "Outage minimization and optimum power control for the fading relay channels," Oct. 2004, vol. 3, pp. 458-462.

[42] M. Hasna and M. Alouini, "Optimal power allocation for relayed transmissions over Rayleigh-fading channels," IEEE Trans. Wireless Comm., vol. 3, no. 6, pp. 1999-2004, Nov. 2004. 
[43] Y. Cao, B. Vojcic, and M. Souryal, "User-cooperative transmission with channel feedback in slow fading environment," in Proc. IEEE Veh. Tech. Conf. (VTC), Sept. 2004, vol. 3, pp. 2063-2067.

[44] R. Annavajjala, P. C. Cosman, and L. B. Milstein, "Statistical knowledge-based optimum power allocation for relaying protocols in the high-SNR regime," IEEE J. Select. Areas Commun., vol. 25, no. 2, Feb. 2007.

[45] D. Gunduz and E. Erkip, "Opportunistic cooperation by dynamic resource allocation," IEEE Trans. Wireless Comm., vol. 6, no. 4, April 2007.

[46] J. Luo, R. S. Blum, L. J. Cimini, L. J. Greenstein, and A. M. Haimovich, "Decodeand-forward cooperative diversity with power allocation in wireless networks," in Proc. IEEE Global Telecommun. Conf. (GLOBECOM), Dec. 2005, vol. 5.

[47] K. Vardhe, D. Reynolds, and M. C. Valenti, "The performance of multi-user cooperative diversity in an asynchronous CDMA uplink," vol. 7, no. 5, pp. 1930-1940, May 2008.

[48] D. P. Bertsekas, Constrained Optimization and Lagrange Multiplier Methods, Academic Press, 1996.

[49] X. Bao, and J. Li, "On the Outage Properties of Adaptive Network Coded Cooperation (ANCC) in Large Wireless Networks," in Proc. Int. Conf. on Acoustics, Speech and Signal Processing (ICASSP), Toulouse, France, May 2006.

[50] N. Laneman, D. Tse, and G. Wornell,, "Cooperative diversity in wireless networks: efficient protocols and outage behavior," IEEE Trans. Inform. Theory, vol. 50, no. 12, pp. 3062-3080, Dec. 2004.

[51] L. Venturino, X. Wang, and M. Lops, "Multiuser detection for cooperative networks and performance analysis," IEEE Trans. Signal Proc., vol. 54, no. 9, pp. 3315-3329, Sept. 2006.

[52] S. Wei, D. Goeckel, and M.C. Valenti, "Asynchronous cooperative diversity," IEEE Trans. Wireless Comm., vol. 5, pp. 375-391, April 2006.

[53] A. Scaglione and Y. W. Hong, "Opportunistic large arrays : Cooperative transmission in wireless multihop ad hoc networks to reach far distances," IEEE Trans. Signal Proc., vol. 51, pp. 2082-2092, August 2003.

[54] Tarik Ganim and M.C. Valenti, "The throughput of hybrid ARQ in block fading under modulation constraints," in Conf. on Inform. Sciences and Sys. (CISS), Pinceton, NJ, Mar. 2006, vol. 3.

[55] S. B. Wicker, Error control systems for digital communication and storage, PrenticeHall, 1996.

[56] H. Dai, S. Jayaweera, H. V. Poor, D. Reynolds, and X. Wang, "Receiver structures for multiuser MIMO systems," a book chapter in MIMO Wireless Communications, Cambridge University Press, 2006. 
[57] X. Wang and H.V. Poor, "Blind adaptive multiuser detection in multipath CDMA channels based on subspace tracking," IEEE Trans. Signal Proc., vol. 46, no. 11, pp. 3030-3044, Nov. 1998.

[58] P. Gupta and P. R. Kumar, "The capacity of wireless networks," IEEE Trans. Inform. Theory, vol. 46, no. 2, pp. 388-404, March 2000.

[59] M. Grossglauser and D. N. C. Tse, "Mobility increases the capacity of ad-hoc wireless networks," IEEE Trans. Networking, vol. 10, no. 4, pp. 477-486, Aug. 2002.

[60] M. O. Hasna, and M. Alouini, "Outage probability of multihop transmission over Nakagami fading channels," IEEE Commun. Letters, vol. 7, no. 5, pp. 216-218, May 2003.

[61] J. Boyer, D. Falconer, and H. Yanikomeroglu, "Multihop diversity in wireless relaying channels," IEEE Trans. Commun., vol. 52, no. 10, pp. 1820-1830, Oct. 2004.

[62] G. Farhadi and N. C. Beaulieu, "Selective decode-and-forward relaying scheme for multihop diversity transmission systems," in Proc. IEEE Global Telecommun. Conf. (GLOBECOM), Nov. 2007, pp. 4385-4390.

[63] M. Zorzi and R. R. Rao, "Geographic random forwarding (GeRaF) for ad hoc and sensor networks: Multihop performance," IEEE Trans. Mobile Comp., vol. 2, pp. 337348, Oct. 2003.

[64] B. Zhao and M. C. Valenti, "Practical relay networks : a generalization of hybrid ARQ," IEEE J. Select. Areas Commun., vol. 23, no. 1, pp. 7-18, Jan. 2005.

[65] S. V. Amari and R. B. Misra, "Closed-form for distribution of sum of exponential random variables," IEEE Trans. Reliability, vol. 46, no. 4, pp. 519-522, Dec. 1997. 


\section{A Convex Optimization and Lagrange Multipliers}

\section{A.1 Convex Sets}

Let $\mathcal{S}$ be a set in real or complex vector space. $\mathcal{S}$ is said to be a convex set if

$$
\theta x+(1-\theta) y \in \mathcal{S}
$$

for all $x, y \in \mathcal{S}$ and $0 \leq \theta \leq 1$. In other words, $\mathcal{S}$ is convex if every point of the line that connects points $x$ and $y$ is also in $\mathcal{S}$.

\section{A.2 Convex Functions}

A real valued function $f$ is called convex if its domain set $\mathcal{S}$ is a convex set and

$$
f(\theta x+(1-\theta) y) \leq \theta f(x)+(1-\theta) f(y)
$$

for all $x, y \in \mathcal{S}, 0 \leq \theta \leq 1$.

- $f$ is concave if $-f$ is convex.

- $f$ is strictly convex if domain of $f$ is convex and

$$
f(\theta x+(1-\theta) y)<\theta f(x)+(1-\theta) f(y)
$$

for all $x, y \in \mathcal{S}, 0 \leq \theta \leq 1$.

- Sum $f_{1}+f_{2}$ is convex if $f_{1}$ and $f_{2}$ are both convex functions.

- $f(A x+B)$ is convex if $f$ is convex.

- Affine functions namely functions of the form $f(x)=A x+B$ are both convex and concave on $\Re$ for any $A, B \in \Re$. 


\section{A.3 Constrained Optimization}

The optimization problem under some constraints can be expressed in the following form:

$$
\begin{aligned}
\operatorname{minimize} & f(\boldsymbol{x}) \\
\text { subject to } & h_{1}(\boldsymbol{x})=0, \\
& \vdots \\
& h_{m}(\boldsymbol{x})=0, \\
& g_{1}(\boldsymbol{x}) \leq 0, \\
& \vdots \\
& g_{p}(\boldsymbol{x}) \leq 0, \\
& \boldsymbol{x} \in \Omega,
\end{aligned}
$$

for $\Omega \in \mathbb{R}^{n}$. The constraints $h_{i}(\boldsymbol{x})=0$ are called equality constraints and the constraints $g_{i}(\boldsymbol{x})$ are inequality constraints. A point $\boldsymbol{x} \in \Omega$ that satisfies all of the functional constraints is said to be feasible. A point $\boldsymbol{x}$ is the solution to the optimization problem if $\boldsymbol{x}$ is feasible and $\boldsymbol{x}$ minimizes $f(\boldsymbol{x})$.

\section{A.4 Lagrange Multiplier}

Lagrange multipliers are a way to solve constrained convex optimization problem. The Lagrange multiplier theorem lets us translate the original constrained optimization problem into an ordinary system of simultaneous equations with introduction of extra variables called Lagrange multipliers. In the above stated constrained optimization problem, at a point of constrained extremum $\boldsymbol{x}^{*}$, the gradient $\nabla f\left(\boldsymbol{x}^{*}\right)$ is parallel to each gradient $\nabla h_{i}(\boldsymbol{x}), i=1,2, \cdots, m$ and $\nabla g_{i}(\boldsymbol{x}), i=1,2, \cdots, p$, so that there exists constants $\lambda_{i}$ such that

$$
\nabla f\left(\boldsymbol{x}^{*}+\lambda_{i} \nabla h_{i}\left(\boldsymbol{x}^{*}\right)\right)+\lambda_{j} \nabla g_{i}\left(\boldsymbol{x}^{*}\right)=0, \quad i=1,2, \cdots, \text { and } j=1,2, \cdots, p \text {. }
$$

Since these hold simultaneously, they hold in the sum, 


$$
\nabla f\left(\boldsymbol{x}^{*}\right)+\sum_{i=1}^{m} \lambda_{i} \nabla h_{i}(\boldsymbol{x} a s t)+\sum_{j=1}^{p} g_{i}\left(\boldsymbol{x}^{*}\right)=0 .
$$

The constants $\lambda_{i}$ are called Lagrange multipliers. Solving the above simultaneous equations yields the minima of the objective function. 


\section{Contributions}

\section{Journal Publications}

1. K. Vardhe and D. Reynolds, "The performance of multistage cooperation in relay networks," in preparation.

2. K. Vardhe, D. Reynolds, and B. D. Woerner, "Joint power allocation and relay selection for multiuser cooperative communication," submitted (revised) IEEE Trans. Wireless Commun., July 2008.

3. K. Vardhe, D. Reynolds, and M. C. Valenti, "The performance of multi-user cooperative diversity in an asynchronous CDMA uplink", IEEE Trans. Wireless Commun., vol. 7, no. 5, pp. 1930-1940, May 2008.

4. K. Vardhe, and D. Reynolds, "User cooperation in an asynchronous cellular uplink", in Signal Processing, vol. 87, no. 7, pp. 1799-1807, July 2007.

\section{Conference Publications}

1. K. Vardhe and D. Reynolds, "The performance of multistage cooperation," submitted to Global Telecom. Conference, (Globecom), (Honolulu, Hawaii), Nov. 2009.

2. K. Vardhe, D. Reynolds, and M. C. Valenti, "Outage probability of a multi-user cooperation protocol in an asynchronous CDMA cellular uplink", in Proc. Conf. on Info. Sci. and Sys. (CISS), (Baltimore, MD), Mar. 2007.

3. K. Vardhe and D. Reynolds, "The performance of space-time coded cooperative diversity in an asynchronous cellular uplink", in Proc. IEEE Military Commun. Conference, (MILCOM), Washington DC, Oct. 2006. 